\title{
ILC as a natural SUSY discovery machine and precision microscope: From light Higgsinos to tests of unification
}

\author{
Howard Baer $\odot,{ }^{1}$ Mikael Berggren $\odot,{ }^{2}$ Keisuke Fujii $\odot,{ }_{5}^{3}$ Jenny List $\odot,{ }^{2, *}$ Suvi-Leena Lehtinen, ${ }^{4,2}$ \\ Tomohiko Tanabe ${ }^{5},{ }^{5}$ and Jacqueline Yan $^{3}$ \\ ${ }^{1}$ University of Oklahoma, Norman, Oklahoma 73019, USA \\ ${ }^{2}$ DESY, Notkestrasse 85, 22607 Hamburg, Germany \\ ${ }^{3}$ High Energy Accelerator Research Organization (KEK), 1-1 Oho, Tsukuba, Ibaraki 305-0801, Japan \\ ${ }^{4}$ Institut für Experimentalphysik, University of Hamburg, Luruper Chaussee 149, 22761 Hamburg, Germany \\ ${ }^{5}$ ICEPP, The University of Tokyo, 7-3-1 Hongo, Bunkyo-ku, Tokyo 113-0033, Japan
}

(Received 19 December 2019; accepted 13 March 2020; published 20 May 2020)

\begin{abstract}
The requirement of electroweak naturalness in simple supersymmetric models implies the existence of a cluster of four light Higgsinos with a mass $\sim 100-300 \mathrm{GeV}$, the lighter the better. While such light compressed spectra may be challenging to observe at the LHC, the International Linear $e^{+} e^{-}$Collider (ILC) with $\sqrt{s}>2 m_{\text {Higgsino }}$ would serve as both a SUSY discovery machine and a precision microscope. We study Higgsino pair production signatures at the ILC based on a full, GEANT4-based simulation of the ILD detector concept. We examine several benchmark scenarios that may be challenging for discovery at the HL-LHC due to mass differences between the Higgsino states between 20 and $4 \mathrm{GeV}$. Assuming $\sqrt{s}=500 \mathrm{GeV}$ and $1000 \mathrm{fb}^{-1}$ of integrated luminosity, the individual Higgsino masses can be measured to $1 \%-2 \%$ precision in the case of the larger mass differences, and at the level of $5 \%$ for the smallest mass difference case. The Higgsino mass splittings are sensitive to the electroweak gaugino masses and allow extraction of gaugino masses to $3 \%-20 \%$ (depending on the model). Extrapolation of gaugino masses via renormalization group running can test the hypothesis of gaugino mass unification. We also examine a case with natural generalized mirage mediation, where the unification of gaugino masses at an intermediate scale apparently gives rise to a natural SUSY spectrum somewhat beyond the reach of HL-LHC.
\end{abstract}

DOI: 10.1103/PhysRevD.101.095026

\section{INTRODUCTION}

The Standard Model (SM) of particle physics has been spectacularly confirmed across a broad array of measurements and often to a very high precision at the LHC. The crowning achievement was to establish the existence of a physical scalar (Higgs) boson with a mass $m_{h}=$ $125.09 \pm 0.24 \mathrm{GeV}[1]$. In spite of this impressive success, the narrative brings with it a cause for concern: quantum mechanical contributions to the Higgs mass rapidly exceed $m_{h}$ for energy fluctuations of an order $\Lambda \sim 1 \mathrm{TeV}$ [2]. These quadratic divergences necessitate ever more incredulous fine-tunings to maintain $m_{h} \simeq 125 \mathrm{GeV}$ as the excluded energy scale of new physics increases. In addition, the SM is lacking the necessary ingredients to explain, e.g., cosmic inflation, the existence of dark

*jenny.list@desy.de

Published by the American Physical Society under the terms of the Creative Commons Attribution 4.0 International license. Further distribution of this work must maintain attribution to the author(s) and the published article's title, journal citation, and DOI. Funded by SCOAP ${ }^{3}$. matter and dark energy in the Universe, the origin of the matter-antimatter asymmetry, and a suppression of $C P$ violation in the strong interactions.

A rather minimal extension of the SM-moving from the Poincare group to the more general super-Poincaré group (supersymmetry or SUSY) of space-time symmetriesallows for solutions or improvements of all these problems. The added spacetime supersymmetry guarantees the cancellation of the offending quadratic divergences to all orders in perturbation theory, thus, rendering the Higgs field natural. The allowance for a vast assortment of scalar fields in SUSY, as expected from string theory, allows for many possible inflaton candidate fields and for a nonzero minimum of the scalar potential, yielding a cosmological constant. The lightest SUSY particle and/or the inclusion of an axion (necessary for solving the strong $C P$ problem) yields dark matter, while scalar field flat direction (AffleckDine) baryogenesis and various other thermal and nonthermal leptogenesis mechanisms seem automatic in SUSY. In addition, SUSY receives indirect support from (1) the measured values of gauge couplings, which unify under minimal supersymmetric standard model (MSSM) renormalization group evolution, (2) the measured value of the top 
mass, which is just right to produce a radiative breakdown of electroweak gauge symmetry, and (3) the measured value of $m_{h} \simeq 125 \mathrm{GeV}$, which lies squarely within the prediction of $m_{h} \lesssim 135 \mathrm{GeV}$ required by the MSSM. ${ }^{1}$

In spite of these theoretical successes, many physicists have developed a large degree of skepticism regarding the eventual emergence of SUSY at experimental facilities. This arises due to (1) the lack of evidence for superpartners at LHC and (2) the rather large value of $m_{h}$ that has been found. The first of these is exemplified by the latest gluino mass limits: that $m_{\tilde{g}} \gtrsim 2 \mathrm{TeV}$ in many simplified models, which may be compared against early projections by Barbieri-Giudice (BG) $[7,8]$, where electroweak naturalness requires $m_{\tilde{g}} \lesssim 400 \mathrm{GeV}$ for fine-tuning parameter $\Delta_{\mathrm{BG}} \lesssim 30$. Secondly, a value of $m_{h} \simeq 125 \mathrm{GeV}$ requires [3] within the MSSM the existence of highly mixed (large trilinear soft parameters $A_{t}$ ) TeV-scale top squarks $\tilde{t}_{1,2}{ }^{2}$ This may be contrasted with Dimopoulos-Giudice naturalness [9] that $m_{\tilde{t}_{1}} \lesssim 350 \mathrm{GeV}$ for $\Delta_{\mathrm{BG}}<30$ or that $m_{\tilde{t}_{1,2}, \tilde{b}_{1}} \lesssim$ $500 \mathrm{GeV}$ from requiring $\delta m_{H_{u}}^{2} \lesssim m_{h}^{2}[10,11]$. Thus, in the LHC era, the question of electroweak naturalness has been elevated to one of prime importance, which can serve as a guide for construction of future experimental facilities. ${ }^{3}$

The most direct connection between the weak scale, as exemplified by the weak gauge and Higgs boson masses $m_{W, Z, h}$ and the SUSY Lagrangian parameters, arises from the scalar potential minimization condition, [13]

$$
\begin{aligned}
\frac{1}{2} m_{Z}^{2} & =\frac{\left(m_{H_{d}}^{2}+\Sigma_{d}^{d}\right)-\left(m_{H_{u}}^{2}+\Sigma_{u}^{u}\right) \tan ^{2} \beta}{\left(\tan ^{2} \beta-1\right)}-\mu^{2} \\
& \simeq-m_{H_{u}}^{2}-\Sigma_{u}^{u}-\mu^{2},
\end{aligned}
$$

where the latter partial equality arises for moderate to large values of the ratio of Higgs vacuum expectation values $\tan \beta \equiv v_{u} / v_{d}$. The $\mu$ term arises as a mass term in the MSSM superpotential; thus, it is supersymmetry conserving and feeds mass both to the SM particles $W, Z$, and $h$ and also the SUSY Higgsinos. The weak scale soft SUSY

\footnotetext{
${ }^{1}$ We quote the bound $m_{h} \lesssim 135 \mathrm{GeV}$ given in Ref. [3], which applies for an assumed soft breaking scale $m_{\text {soft }} \lesssim 2 \mathrm{TeV}$. The light Higgs mass bound increases logarithmically with increasing $m_{\text {soft }}$ and can reach as high as $m_{h} \lesssim 140-155 \mathrm{GeV}$ for models of split SUSY [4,5]. Such models with very high values of $m_{\text {soft }}$ are highly fine-tuned under $\Delta_{\mathrm{EW}}$. For our case of natural SUSY with $\Delta_{\mathrm{EW}}<30$, then the top squark mass $m_{\tilde{t}_{1}}$ is bounded from above by $\sim 3 \mathrm{TeV}$ [due to the $\Sigma_{u}^{u}\left(\tilde{t}_{1,2}\right)$ terms in Eq. (1)] leading to $m_{h_{2}} \lesssim 127 \mathrm{GeV}[6]$.

${ }^{2}$ A value of $m_{h} \sim 125 \mathrm{GeV}$ can also be obtained with small $A_{t}$ but with huge (unnatural) top-squark soft terms. For our case of natural SUSY with $\Delta_{\mathrm{EW}}<30$ and $m_{h} \simeq 125 \mathrm{GeV}$, then a large $A_{t}$ at the weak scale is required [6].

${ }^{3}$ In Ref. [12], it is declared that "settling the ultimate fate of naturalness is perhaps the most profound theoretical question of our time ... and will largely dictate the future of fundamental physics in this century."
}

breaking term $m_{H_{u}}^{2}$ feeds mass just to $W, Z$, and $h$ (and other SUSY Higgs via suppressed mixing). The $\Sigma_{u}^{u}$ are radiative corrections (for a full listing, see Ref. [6]); the largest of which typically arise from the top-squark contributions. The MSSM may be considered as natural if there are no large, unnatural cancellations (fine-tunings) on the right-hand side of Eq. (1). A naturalness measure $\Delta_{\mathrm{EW}}$ has been proposed which considers the ratio of the largest element on the right-hand side (rhs) of Eq. (1) to $m_{Z}^{2} / 2$. The fine-tuning of $m_{Z}$ sets in for values of $\Delta_{\mathrm{EW}} \gtrsim 20-30$ and is visually displayed in Fig. 1 of Ref. [14].

The validity of the early naturalness estimates using the BG measure has been challenged in that these calculations are performed using multiple-soft-parameter effective theories instead of more fundamental theories in which the soft parameters are all related [15]. ${ }^{4}$ Using correlated soft parameters, the BG measure reduces to the EW measure [17]. The validity of naturalness estimates using $\delta m_{H_{u}}^{2} / m_{h}^{2}$ has been challenged in that, in an effort to simplify, several contributions to $m_{h}^{2}$ and $\delta m_{H_{u}}^{2}$ have been set to zero. By including these pieces, then one allows for radiatively driven naturalness (RNS) [18] wherein large, seemingly unnatural high scale values of $m_{H_{u}}^{2}$ are driven to natural values at the weak scale. The revised measure is thus brought into accord with $\Delta_{\mathrm{EW}}[15,17]$.

From Eq. (1), the requirements for electroweak naturalness are then

(i) The superpotential $\mu$ parameter, bounded from below by $\mu \gtrsim 100 \mathrm{GeV}$ due to chargino searches at LEP2, is not too far from $m_{Z}: \mu \sim 100-300 \mathrm{GeV}$, the lower the better. This immediately implies the existence of several Higgsino-like electroweakinos in SUSY with $m_{\tilde{\chi}_{1}^{ \pm}} \sim m_{\tilde{\chi}_{1,2}^{0}} \sim|\mu|{ }^{5}$

(ii) The soft term $m_{H_{u}}^{2}$, which must be driven to negative values to initiate a breakdown of electroweak symmetry, is driven to small and not large negative values.

\footnotetext{
${ }^{4}$ For example, in the string motivated SUSY model with dominant soft breaking from the dilaton, then one expects $m_{0}=m_{3 / 2}, m_{1 / 2}=-A_{0}=\sqrt{3} m_{3 / 2}$, where $m_{3 / 2}$ is the gravitino mass. In such a top-down case, it does not make sense to use $m_{0}$, $m_{1 / 2}$, and $A_{0}$ as independent parameters, and one obtains a very different value of $\Delta_{\mathrm{BG}}$. Alternatively, in the string landscape picture, then there is a statistical pull to large soft terms balanced by the anthropic requirement of a not-to-large value of the weak scale in pocket universes within the multiverse. In such a case, the multiverse selects soft terms consistent with radiatively driven naturalness, where $m_{h}$ is pulled to $125 \mathrm{GeV}$ whilst sparticle masses are pulled beyond LHC bounds [16].

${ }^{5}$ It is possible that nonholomorphic soft terms may arise allowing for higher mass Higgsinos without compromising naturalness [19]. Such "semisoft" mass terms are expected to be of order $m_{\text {weak }}^{2} / m_{P}$ [20] but in the case where the mediation scale is arranged to be far lower than the usual Planck scale (as expected for gravity mediation), then these terms can become much larger.
} 


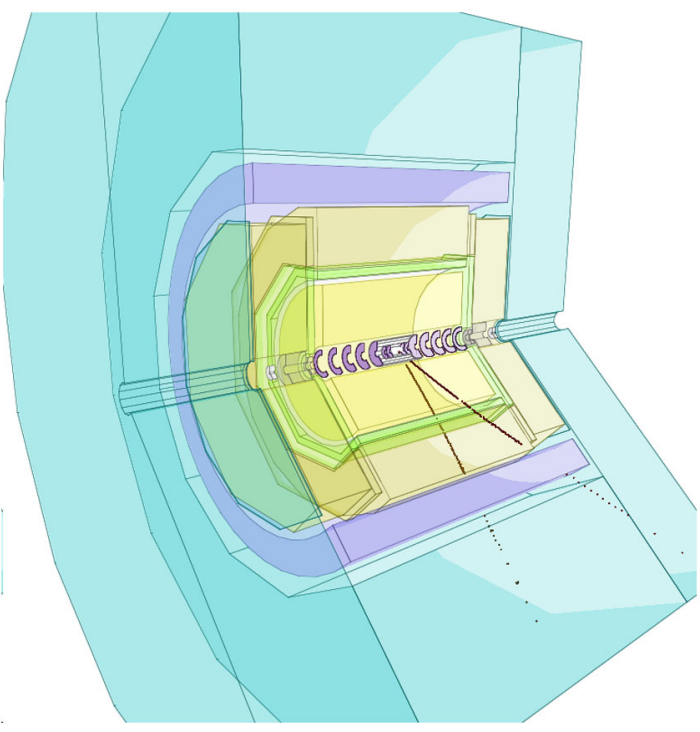

FIG. 1. ILD event display of a simulated $e^{+} e^{-} \rightarrow \tilde{\chi}_{1}^{0} \tilde{\chi}_{2}^{0}$ event with $\tilde{\chi}_{2}^{0} \rightarrow \mu^{+} \mu^{-} \tilde{\chi}_{1}^{0}$.

(iii) The radiative corrections $\Sigma_{u}^{u}\left(\tilde{t}_{1,2}\right)$ are actually minimized for $\mathrm{TeV}$-scale highly mixed top squarks. These same conditions lift the Higgs mass to $m_{h} \simeq 125 \mathrm{GeV}$. Detailed evaluations require $m_{\tilde{t}_{1}} \lesssim$ $3 \mathrm{TeV}$ for $\Delta_{\mathrm{EW}}<30[6]$.

(iv) The gluino mass contributes at a two-loop level to Eq. (1) via the $\Sigma_{u}^{u}\left(\tilde{t}_{1,2}\right)$. Detailed evaluations require $m_{\tilde{g}} \lesssim 6 \mathrm{TeV}$ for $\Delta_{\mathrm{EW}}<30$. $^{6}$ This may be compared to the ultimate reach of the HL-LHC, which extends to about $m_{\tilde{g}} \sim 2.8 \mathrm{TeV}$ (at the $5 \sigma$ discovery level [22]).

Thus, the HL-LHC will probe only a portion of natural SUSY parameter space via gluino and top squark pair production searches.

The naturalness-required light Higgsinos may be produced at decent rates at LHC, but their relatively compressed spectra imply low visible energy release from their decays. Thus, light Higgsinos are very challenging to see at LHC $[22,23]$. In contrast, the International Linear $e^{+} e^{-}$Collider (ILC) with $\sqrt{s}>2 m$ (Higgsino) would be a Higgsino factory in addition to being a Higgs factory. The reactions $e^{+} e^{-} \rightarrow \tilde{\chi}_{1}^{+} \tilde{\chi}_{1}^{-}$and $\tilde{\chi}_{2}^{0} \tilde{\chi}_{1}^{0}$ should occur at rates comparable to muon pair production and at rates exceeding $Z h$ production [24]. The expected mass gaps $m_{\tilde{\chi}_{1}}-m_{\tilde{\chi}_{1}^{0}} \sim m_{\tilde{\chi}_{2}^{0}}-m_{\tilde{\chi}_{1}^{0}} \sim$ 3-20 GeV lead to events, which are easily identified at ILC: see Fig. 1 for a simulated $e^{+} e^{-} \rightarrow \tilde{\chi}_{1}^{0} \tilde{\chi}_{2}^{0}$ with $\tilde{\chi}_{2}^{0} \rightarrow \mu^{+} \mu^{-} \tilde{\chi}_{1}^{0}$ event display with light Higgsinos in the ILD detector.

The cleanliness of ILC Higgsino pair production events along with tunable beam energy and beam polarization should allow for a rich program of Higgsino measurements. While the Higgsino masses should be comparable to the

\footnotetext{
${ }^{6}$ In the case of natural anomaly mediated SUSY breaking [21], the gluino mass bound increases to $m_{\tilde{g}} \lesssim 9 \mathrm{TeV}$.
}

superpotential $\mu$ parameter, thus allowing for a determination of $\mu$, the Higgsino mass splittings depend sensitively on the weak scale gaugino masses $M_{1}$ (bino) and $M_{2}$ (wino). Thus, precision measurements of $m_{\tilde{\chi}_{1}^{ \pm}}$and $m_{\tilde{\chi}_{1.2}^{0}}$ should allow for an extraction of $M_{1}$ and $M_{2}$ to good precision. Once the soft breaking gaugino masses are known, then the physical masses of the heavier neutralinos and charginos can also be found. The fitted values of $M_{1}$ and $M_{2}$ can be extrapolated to high energies to test the hypothesis of gaugino mass unification. If gluinos are discovered at LHC, then $M_{3}$ (gluino) may be extracted [25] and unification of all three gaugino masses may be tested.

In this paper, we first present in Sec. II two natural SUSY benchmark models labeled ILC1 and ILC2 that arise from the nonuniversal Higgs model (NUHM2)[26]. These models allow for $\mu$ as an input parameter so that SUSY spectra with a low value of $\Delta_{\mathrm{EW}}$ can easily be generated. The NUHM2 model incorporates gaugino mass unification so that under the renormalization group (RG) evolution, $M_{1}$ and $M_{2}$ should unify at the scale $m_{\mathrm{GUT}} \simeq 2 \times 10^{16} \mathrm{GeV}$. We also propose a natural generalized mirage mediation (nGMM) benchmark model, which instead has gaugino mass unification at the mirage scale $\mu_{\text {mir }}=m_{\mathrm{GUT}} e^{-8 \pi^{2} / \alpha} \sim$ $5 \times 10^{7} \mathrm{GeV}$, where $\alpha=4$ parametrizes the relative amounts of modulus mediation versus anomaly mediation in SUSY breaking. In this case, by determining the mirage unification scale $\mu_{\text {mir }}$, ILC can measure the strength $\alpha$ of moduli vs anomaly mediation. If the gaugino masses are extrapolated beyond the mirage scale to the GUT scale, then ILC can also indirectly measure the underlying gravitino mass $m_{3 / 2}$ [27]. Thus, in such cases, ILC would allow for a window into the nature of the laws of physics at energy scales far beyond $\sqrt{s} \sim 0.5-1 \mathrm{TeV}$.

All three benchmarks have been studied in a detailed, GEANT4-based simulation of the ILD detector concept [28], which we introduce in Sec. III. In Sec. IV, we present a detailed portrait of various Higgsino pair production measurements at ILC with $\sqrt{s}=500 \mathrm{GeV}$. Continuum measurements of energy and invariant mass distributions of Higgsino decay products should allow for extraction of Higgsino masses to percent level or better precision.

In Sec. V, we present results from our calculations using the FITTINO [29] program to extract fits of fundamental weak scale MSSM Lagrangian parameters, especially the gaugino masses $M_{1}$ and $M_{2}$. We also obtain predictions for the masses of many of the kinematically inaccessible superparticles. We then can extract the underlying GUT scale parameters if we assume a particular high scale SUSY model such as NUHM2, NUHM3, or nGMM. If the gluino is discovered at LHC, the extracted gaugino masses may be augmented with the $S U(3)$ gaugino mass $M_{3}$. We also show that the thermally produced relic density of Weakly Interacting Massive Particles (WIMPs) may be extracted, thus testing the WIMP only versus mixed axion-WIMP dark matter hypotheses. In Sec. VI, we show results from 
running the gaugino masses to high energy scales, thus offering a test of the unification hypothesis and the underlying SUSY breaking mechanism. A summary and conclusions are presented in Sec. VII.

\section{BENCHMARK MODELS}

In this section, we present three natural SUSY benchmark points, which have been used for the detailed ILD studies described in Secs. III and IV.

\section{A. ILC1 benchmark model}

The ILC1 benchmark point, whose parameters are listed in Table I, has been presented previously [18] and has been used for some detailed ILC studies using a toy detector simulation [24]. The ILC1 benchmark point was generated within the NUHM2 model with input parameters and output masses as listed. While the various matter scalars are essentially decoupled for LHC and ILC physics, the spectrum does contain light Higgsinos with mass $m_{\tilde{\chi}_{1}^{ \pm}}=117.3 \mathrm{GeV}$ and $m_{\tilde{\chi}_{1.2}^{0}}=102.7$ and $124 \mathrm{GeV}$, respectively, so that Higgsino pair production should already turn on for ILC with $\sqrt{s}>227 \mathrm{GeV}$. The associated mass gaps, which play a central role in these analyses, are $m_{\tilde{\chi}_{1}^{ \pm}}-m_{\tilde{\chi}_{1}^{0}}=14.6 \mathrm{GeV}$ and $m_{\tilde{\chi}_{2}^{0}}-m_{\tilde{\chi}_{1}^{0}}=21.3 \mathrm{GeV}$. The model contains a light Higgs scalar with $m_{h}=125.3 \mathrm{GeV}$ due to highly mixed TeV-scale top squarks with $m_{\tilde{t}_{1}}=1893.3 \mathrm{GeV}$ (well beyond the reach of HL-LHC [30], where the 95\% C.L. exclusion reach extends to $m_{\tilde{t}_{1}} \sim 1400 \mathrm{GeV}$ [31]). The model is highly

TABLE I. Input parameters and mass spectrum and rates for benchmark points ILC1, ILC2, and nGMM1. All masses and dimensionful parameters are in $\mathrm{GeV}$ units. All values have been obtained with ISASUGRA.

\begin{tabular}{|c|c|c|c|c|}
\hline & Units & ILC1 & ILC2 & nGMM1 \\
\hline$M_{0}$ & {$[\mathrm{GeV}]$} & 7025.0 & 5000 & . \\
\hline$M_{1 / 2}$ & {$[\mathrm{GeV}]$} & 568.3 & 1200 & $\cdots$ \\
\hline$A_{0}$ & {$[\mathrm{GeV}]$} & -10427 & -8000 & $\cdots$ \\
\hline$m_{3 / 2}$ & {$[\mathrm{GeV}]$} & $\cdots$ & $\cdots$ & 75000 \\
\hline$M_{1}, M_{2}, M_{3}$ & {$[\mathrm{GeV}]$} & $\cdots$ & $\cdots$ & $3382.5,2124.4,1225.8$ \\
\hline $\tan \beta$ & $\ldots$ & 10 & 15 & 10 \\
\hline$a_{3}$ & $\cdots$ & $\cdots$ & $\cdots$ & 3 \\
\hline$c_{m}$ & $\cdots$ & $\cdots$ & $\cdots$ & 6.9 \\
\hline$\alpha$ & $\cdots$ & $\cdots$ & $\cdots$ & 4 \\
\hline$m_{h}$ & {$[\mathrm{GeV}]$} & 125.3 & 125.4 & 124.9 \\
\hline$m_{A}$ & {$[\mathrm{GeV}]$} & 1000.0 & 1000 & 2000 \\
\hline$m_{H}$ & {$[\mathrm{GeV}]$} & 1006.8 & 1006.7 & 2013.3 \\
\hline$m_{H^{ \pm}}$ & {$[\mathrm{GeV}]$} & 1003.2 & 1003.2 & 2001.6 \\
\hline$\mu$ & {$[\mathrm{GeV}]$} & 115.0 & 150 & 150 \\
\hline$m_{\tilde{g}}$ & {$[\mathrm{GeV}]$} & 1563.5 & 2832.6 & 2856.5 \\
\hline$m_{\tilde{\chi}_{1}^{ \pm}}, m_{\tilde{\chi}_{2}^{ \pm}}$ & {$[\mathrm{GeV}]$} & $117.3,513.0$ & $158.3,1017.5$ & $158.7,1791.6$ \\
\hline$m_{\tilde{\chi}_{1}^{0}}, m_{\tilde{\chi}_{2}^{0}}$ & {$[\mathrm{GeV}]$} & $102.7,124.0$ & $148.1,157.8$ & $151.4,155.8$ \\
\hline$m_{\tilde{\chi}_{3}^{0}}, m_{\tilde{\chi}_{4}^{0}}$ & {$[\mathrm{GeV}]$} & $267.0,524.2$ & $538.7,1031.1$ & $1526.9,1799.4$ \\
\hline$m_{\tilde{u}_{L}}, m_{\tilde{u}_{R}}$ & {$[\mathrm{GeV}]$} & 7021, 7254 & 5440,5566 & 5267,5399 \\
\hline$m_{\tilde{t}_{1}}, m_{\tilde{t}_{2}}$ & {$[\mathrm{GeV}]$} & 1893,4919 & 1774,3878 & 1433,3732 \\
\hline$m_{\tilde{d}_{L}}, m_{\tilde{d}_{R}}$ & {$[\mathrm{GeV}]$} & 7022, 6999 & 5441,5384 & 5267,5229 \\
\hline$m_{\tilde{b}_{1}}, m_{\tilde{b}_{2}}$ & {$[\mathrm{GeV}]$} & 4959,6893 & 3903,5204 & 3770,5124 \\
\hline$m_{\tilde{e}_{L}}, m_{\tilde{e}_{R}}$ & {$[\mathrm{GeV}]$} & 7152,6759 & 5149,4817 & 5128,4825 \\
\hline$m_{\tilde{\tau}_{1}}, m_{\tilde{\tau}_{2}}$ & {$[\mathrm{GeV}]$} & 6657,7103 & 4652,5072 & 4749,5094 \\
\hline$\Omega_{\tilde{\chi}^{0}}^{\mathrm{TP}} h^{2}$ & $\cdots$ & 0.009 & 0.007 & 0.005 \\
\hline$\langle\sigma v\rangle(v \rightarrow 0)$ & {$\left[\mathrm{cm}^{3} \mathrm{~s}^{-1}\right]$} & $2.2 \times 10^{-25}$ & $2.9 \times 10^{-25}$ & $3.1 \times 10^{-25}$ \\
\hline$\sigma^{\mathrm{SI}}\left(\tilde{\chi}^{0} p\right) \times 10^{9}$ & {$[\mathrm{pb}]$} & 6.8 & 1.5 & 0.3 \\
\hline$a_{\mu}^{\mathrm{SUSY}} \times 10^{10}$ & $\cdots$ & 0.03 & 0.13 & 0.06 \\
\hline$B F(b \rightarrow s \gamma) \times 10^{4}$ & $\cdots$ & 3.3 & 3.3 & 3.1 \\
\hline$B F\left(B_{S} \rightarrow \mu^{+} \mu^{-}\right) \times 10^{9}$ & $\cdots$ & 3.8 & 3.8 & 3.8 \\
\hline$B F\left(B_{u} \rightarrow \tau \nu_{\tau}\right) \times 10^{4}$ & $\cdots$ & 1.3 & 1.3 & 1.3 \\
\hline$\Delta_{\mathrm{EW}}$ & $\cdots$ & 14 & 28 & 15 \\
\hline
\end{tabular}


electroweak natural with $\Delta_{\mathrm{EW}}=14$ corresponding to just 7\% fine-tuning. This benchmark is now likely excluded by LHC: The gluino mass $m_{\tilde{g}}=1563.5 \mathrm{GeV}$ is excluded by LHC13 searches with $\sim 36 \mathrm{fb}^{-1}$. The searches for Higgsinos with small mass-splittings performed by CMS [32] and ATLAS [33] are done for a spectrum different from our benchmarkin particular, the mass of $\tilde{\chi}_{1}^{ \pm}$is assumed to be exactly halfway between those of $\tilde{\chi}_{1}^{0}$ and $\tilde{\chi}_{2}^{0}$-but are nevertheless likely to exclude it. However, we retain the point for comparison with previous work. If one adopts modest gaugino mass nonuniversality, then a small increase in $M_{3}$ (weak) $>2 \mathrm{TeV}$ would bring the point in accord with LHC searches. The relic density of thermally produced Higgsino-like lightest supersymmetric particles (LSPs) is a factor 13 below the measured value. Requiring also naturalness in the QCD sector, then one must bring the axion into the model, and axionic dark matter may constitute the bulk of the dark matter [34]. The location of the ILC1 benchmark point is denoted by one of the green stars in the $\mu$ vs $M_{1 / 2}$ parameter space plane of the NUHM2 model shown in Fig. 2.

\section{B. ILC2 benchmark}

The ILC2 benchmark point is also generated within the NUHM2 SUSY model with parameter values as listed in

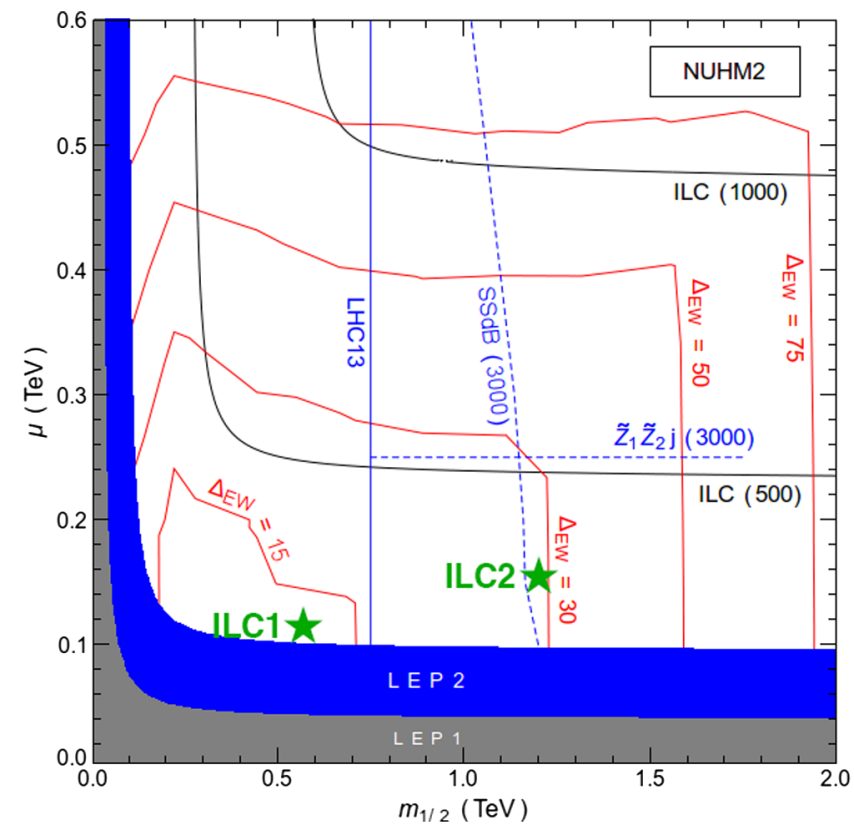

FIG. 2. The $M_{1 / 2}$ vs $\mu$ plane in the NUHM2 model for $\tan \beta=15, M_{0}=5 \mathrm{TeV}, A_{0}=-8 \mathrm{TeV}$, and $M_{A}=1 \mathrm{TeV}$. We show contours of $\Delta_{\mathrm{EW}}$ along with some limits from LHC13 and the future reach of ILC with $\sqrt{s}=500$ and $1000 \mathrm{GeV}$ and HL-LHC [via same-sign diboson production labeled SSdB (3000) and via neutralino associated production labeled $\tilde{Z}_{1} \tilde{Z}_{2} j$ (3000) in the nomenclature of Ref. [35] where $\tilde{Z}_{i}$ denotes neutralino eigenstate $i$ ]. Location of benchmark points is indicated in green. To aid the reader, we note that $m_{\tilde{g}} \sim 2.5 M_{1 / 2}$. The figure is adapted from Ref. [35].
Table I. The location of ILC2 is indicated by the other green star in Fig. 2 and is found to lie just beyond the HL-LHC reach for the same-sign diboson signature arising from the wino pair production [36]. The Higgsino pair signature from $p p \rightarrow \tilde{\chi}_{1}^{0} \tilde{\chi}_{2}^{0} j$ production followed by $\tilde{\chi}_{2}^{0} \rightarrow \tilde{\chi}_{1}^{0} \ell^{+} \ell^{-}$ decay should be viable since the cluster of Higgsinos lies in the vicinity of $\mu=150 \mathrm{GeV}$ [23]. The value of $m_{\tilde{g}}=$ $2832 \mathrm{GeV}$ appears just beyond the HL-LHC reach for gluino pair production (where the $5 \sigma$ reach extends to $m_{\tilde{g}} \sim 2800 \mathrm{GeV}$ [25]). The higher value of gaugino masses in ILC2 - as compared to benchmark point ILC1 1 -is reflected in the reduced inter-Higgsino mass gaps where we find $m_{\tilde{\chi}_{1}^{ \pm}}-m_{\tilde{\chi}_{1}^{0}}=10.2 \mathrm{GeV}$ and $m_{\tilde{\chi}_{2}^{0}}-m_{\tilde{\chi}_{1}^{0}}=$ $9.7 \mathrm{GeV}$. The naturalness measure $\Delta_{\mathrm{EW}}=28$ leads to $\sim 3 \%$ electroweak fine-tuning. The thermally produced abundance of dark matter $\Omega_{\tilde{\chi}_{1}^{0}}^{\mathrm{TP}} h^{2} \sim 0.007$, well below the measured value of 0.12 . So, as for ILC1, the axions needed to solve the strong $\mathrm{CP}$ problem can be expected to make up the remainder.

\section{Natural mirage mediation (NMM) benchmark}

Mirage mediated (MM) SUSY breaking models are motivated by string model compactifications with the moduli fields stabilized by fluxes and where an uplifted scalar potential leads to a de Sitter vacuum (as required by cosmology) with a small breaking of supersymmetry [37-39]. In such cases, it is expected that the SUSY breaking soft terms arise with comparable moduli-mediated and anomaly mediated contributions. In the gaugino sector (and in the scalar sector for particular modular weight choices), the GUT scale soft masses are offset from each other by contributions containing their gauge group beta functions. As a consequence, the running of the gaugino masses exactly compensates the high scale mass splitting leading to an apparent unification at the intermediate (mirage) scale $\mu_{\text {mir }}=m_{\mathrm{GUT}} e^{-8 \pi^{2} / \alpha}$, where the parameter $\alpha$ is introduced to parametrize the relative amounts of anomaly versus moduli mediation. A value $\alpha=0$ corresponds to pure anomaly mediation (with tachyonic sleptons) while, as $\alpha$ gets large, the soft terms become increasingly universal.

Initially, simple MM models predicted scalar masses involving discrete values of scalar field modular weights, which depend on the compactification geometry and upon which branes harbored the various visible sector fields. This class of models, over a wide range of choices for modular weights, was found to be unnatural when $m_{h} \simeq$ $125 \mathrm{GeV}$ was required [17]. However, in more general compactification and stabilization schemes, then the previously discrete parameter choices $c_{m}, c_{H_{u}}, c_{H_{d}}$, and $a_{3}$ become continuous, allowing for the construction of models with low values of $\Delta_{\mathrm{EW}}$ [27].

In Table I, we show one such example point from natural generalized mirage mediation or nGMM in column 4 . The gaugino masses unify at the mirage scale $\mu_{\text {mir }} \sim$ $5 \times 10^{7} \mathrm{GeV}$. The rather large value of $m_{\tilde{g}}=2856.5 \mathrm{GeV}$ 
TABLE II. Higgsino and gaugino fractions of the lightest neutralino $\tilde{\chi}_{1}^{0}$. The fractions are expressed so that they satisfy the relation $R_{\tilde{H}}^{2}+R_{\tilde{W}}^{2}+R_{\tilde{B}}^{2}=1$.

\begin{tabular}{lrcc}
\hline \hline & ILC1 & ILC2 & nGMM1 \\
\hline$R_{\tilde{H}}$ & 0.97 & 0.99 & 0.999 \\
$R_{\tilde{W}}$ & -0.14 & 0.07 & 0.04 \\
$R_{\tilde{B}}$ & 0.19 & 0.08 & 0.02 \\
\hline \hline
\end{tabular}

means the winos and bino are also rather massive so that both gluino pair production and wino pair production appear out of reach of HL-LHC. The Higgsinos are clustered with masses around $\mu \sim 150 \mathrm{GeV}$ but with even smaller mass splittings than ILC2: $m_{\tilde{\chi}_{1}^{ \pm}}-m_{\tilde{\chi}_{1}^{0}}=7.3 \mathrm{GeV}$ and $m_{\tilde{\chi}_{2}^{0}}-m_{\tilde{\chi}_{1}^{0}}=$ $4.4 \mathrm{GeV}$. Such small neutralino mass splittings may also push the soft dilepton plus jet signature from $\tilde{\chi}_{1}^{0} \tilde{\chi}_{2}^{0} j$ production out of reach of the HL-LHC [22]. Nonetheless, the model is highly natural with $\Delta_{\mathrm{EW}}=15$ or $6.7 \%$ fine-tuning. As shown in Table II, the LSP is more purely Higgsino-like than in the ILC1 or ILC2 benchmarks leading to a reduced thermally produced dark matter relic density and reduced direct dark matter detection rates. Thus, the direct detection of WIMP dark matter from the nGMM1 benchmark may require multiton noble liquid detectors for discovery.

\section{SOFTWARE TOOLS AND OBSERVABLES}

In this section, we describe the main features of the ILD detector as used for the simulation study and introduce the characteristics of the Higgsino signal on which the strategies for event selection and reconstruction will be based.

\section{A. Event generation}

The physical masses for the three benchmark points have been calculated by ISASUGRA. The SUSY and SM events have been generated using WHIZARD1.95 [40], which considers both resonant and nonresonant production, as well as their interference. WHIZARD also generates the amount and spectrum of ISR appropriate for each considered channel and takes the beam polarization fully into account. The dedicated setup of the generator provided by the ILC Generator Group was used, and all types $\mathrm{SM} e^{+} e^{-}$ interactions yielding up to six fermions in the final state were considered. In addition, all e $\gamma$ interactions yielding three or five fermions and all $\gamma \gamma$ interactions yielding up to four fermions were also included. The initial photons in the latter cases might be virtual (in which case, the beam remnants come in addition to the final fermions) or real from the photon component of the beams (in which case, there are no beam remnants). The electron and positron beams have an initial energy spread, which is further smeared by the effects of beamstrahlung. The resulting spectra as well as the flux and energy spectra of the beam photons are simulated according to the parameters in the ILC Technical Design Report (TDR) [41], using GUINEAPIG [42].

Pure left-handed or right-handed beam polarizations are used for the event generation. These samples are then weighted according to the nominal ILC beam polarizations for our simulation study. We introduce the following notation for beam polarizations: $\mathcal{P} \equiv\left(\mathcal{P}_{e^{-}}, \mathcal{P}_{e^{+}}\right)$ and define the pure beam polarizations as $\mathcal{P}_{L R} \equiv(-1,+1)$ and $\mathcal{P}_{R L} \equiv(+1,-1)$. The nominal beam polarizations for the ILC are defined as $\mathcal{P}_{-+} \equiv(-0.8,+0.3)$ and $\mathcal{P}_{+-} \equiv(+0.8,-0.3)$.

Table III shows the production cross sections for chargino and neutralino pairs for the three benchmark models introduced in Sec. II A-II C for $100 \%$ polarized beams at several center-of-mass energies. Table IV shows the decay branching ratios in the same three benchmarks.

The results of the simulation study assume a center-ofmass energy of $\sqrt{s}=500 \mathrm{GeV}$ and an integrated luminosity of $\mathcal{L}=500 \mathrm{fb}^{-1}$ for each beam polarization; these results are then scaled according to the operation scenarios

TABLE III. Chargino and neutralino production cross sections for the three benchmark points calculated using WHIZARD at various center-of-mass energies. The ILC beam energy spectrum and ISR effects are included. Pure beam polarizations are assumed.

\begin{tabular}{|c|c|c|c|c|c|}
\hline \multirow[b]{2}{*}{$\sqrt{s}$} & \multirow[b]{2}{*}{ Process } & \multirow[b]{2}{*}{$\mathcal{P}$} & \multicolumn{3}{|c|}{ Cross section $[\mathrm{fb}]$} \\
\hline & & & ILC1 & ILC2 & nGMM1 \\
\hline \multirow[t]{4}{*}{$250 \mathrm{GeV}$} & \multirow{2}{*}{$e^{-} e^{+} \rightarrow \tilde{\chi}_{1}^{+} \tilde{\chi}_{1}^{-}$} & $\mathcal{P}_{L R}$ & 2618 & $\ldots$ & $\cdots$ \\
\hline & & $\mathcal{P}_{R L}$ & 397.1 & $\cdots$ & $\cdots$ \\
\hline & \multirow{2}{*}{$e^{-} e^{+} \rightarrow \tilde{\chi}_{1}^{0} \tilde{\chi}_{2}^{0}$} & $\mathcal{P}_{L R}$ & 1044 & $\cdots$ & $\cdots$ \\
\hline & & $\mathcal{P}_{R L}$ & 804.8 & $\cdots$ & $\cdots$ \\
\hline \multirow[t]{4}{*}{$350 \mathrm{GeV}$} & \multirow{2}{*}{$e^{-} e^{+} \rightarrow \tilde{\chi}_{1}^{+} \tilde{\chi}_{1}^{-}$} & $\mathcal{P}_{L R}$ & 3094 & 1602 & 1571 \\
\hline & & $\mathcal{P}_{R L}^{L \Lambda}$ & 538.8 & 302.8 & 301.4 \\
\hline & \multirow{2}{*}{$e^{-} e^{+} \rightarrow \tilde{\chi}_{1}^{0} \tilde{\chi}_{2}^{0}$} & $\mathcal{P}_{L R}$ & 897.0 & 578.5 & 576.0 \\
\hline & & $\mathcal{P}_{R L}$ & 691.5 & 446.0 & 444.1 \\
\hline \multirow[t]{4}{*}{$500 \mathrm{GeV}$} & \multirow[t]{2}{*}{$e^{-} e^{+} \rightarrow \tilde{\chi}_{1}^{+} \tilde{\chi}_{1}^{-}$} & $\mathcal{P}_{L R}$ & 1801 & 1531 & 1520 \\
\hline & & $\mathcal{P}_{R L}$ & 334.8 & 307.2 & 309.2 \\
\hline & \multirow[t]{2}{*}{$e^{-} e^{+} \rightarrow \tilde{\chi}_{1}^{0} \tilde{\chi}_{2}^{0}$} & $\mathcal{P}_{L R}$ & 491.4 & 458.9 & 463.3 \\
\hline & & $\mathcal{P}_{R L}$ & 379.8 & 353.8 & 357.1 \\
\hline
\end{tabular}

TABLE IV. Chargino and neutralino decay branching ratios for the three benchmark points calculated using ISASUGRA. For the final-state leptons, only the electrons and muons are included $(\ell=e, \mu)$.

\begin{tabular}{lccc}
\hline \hline & ILC1 & ILC2 & nGMM1 \\
\hline $\operatorname{BR}\left(\tilde{\chi}_{1}^{+} \rightarrow \tilde{\chi}_{1}^{0} q q^{\prime}\right)$ & $67 \%$ & $67 \%$ & $66 \%$ \\
$\operatorname{BR}\left(\tilde{\chi}_{1}^{+} \rightarrow \tilde{\chi}_{1}^{0} \ell^{+} \nu_{\ell}\right)$ & $22 \%$ & $22 \%$ & $22 \%$ \\
$\operatorname{BR}\left(\tilde{\chi}_{2}^{0} \rightarrow \tilde{\chi}_{1}^{0} q q^{\prime}\right)$ & $58 \%$ & $63 \%$ & $51 \%$ \\
$\operatorname{BR}\left(\tilde{\chi}_{2}^{0} \rightarrow \tilde{\chi}_{1}^{0} \ell^{+} \ell^{-}\right)$ & $7.4 \%$ & $8.0 \%$ & $7.5 \%$ \\
\hline \hline
\end{tabular}


TABLE V. Total integrated luminosities for various operation scenarios for the ILC [43]. H20 is assumed for ILC1, while I20 is assumed for ILC2 and nGMM1. See Ref. [43] for the assumed timelines and machine upgrades.

\begin{tabular}{lccr}
\hline \hline Scenario & $\sqrt{s}[\mathrm{GeV}]$ & $\mathcal{P}$ & $\mathcal{L}\left[\mathrm{fb}^{-1}\right]$ \\
\hline H20 & 250 & $\mathcal{P}_{-+}$ & 900 \\
& & $\mathcal{P}_{+-}$ & 900 \\
& 350 & $\mathcal{P}_{-+}$ & 90 \\
& & $\mathcal{P}_{+-}$ & 90 \\
& \multirow{2}{*}{500} & $\mathcal{P}_{-+}$ & 1600 \\
& & $\mathcal{P}_{+-}$ & 1600 \\
& \multirow{2}{*}{250} & $\mathcal{P}_{-+}$ & 225 \\
& & $\mathcal{P}_{+-}$ & 225 \\
& 350 & $\mathcal{P}_{-+}$ & 765 \\
& & $\mathcal{P}_{+-}$ & 765 \\
& \multirow{2}{*}{500} & $\mathcal{P}_{-+}$ & 1600 \\
& & $\mathcal{P}_{+-}$ & 1600 \\
\hline \hline
\end{tabular}

listed in Table V for the parameter fit. In the case of ILC1, where all three Higgsinos would already be kinematically accessible at $\sqrt{s}=250 \mathrm{GeV}$, the assumed integrated luminosities correspond to the $\mathrm{H} 20$ operating scenario, while for the other two benchmarks, the I20 scenario was assumed, since in these cases, the Higgsinos are only accessible at $\sqrt{s}=350 \mathrm{GeV}$.

\section{B. The ILD detector model}

The ILD concept is one of the two detectors being designed for the ILC. ILD employs a hybrid tracking system comprised of a time projection chamber and silicon strip sensors for tracking, and silicon pixel sensors as vertex detectors. Outside of the tracking system sits a highly granular calorimeter system optimized for particle flow reconstruction. A superconducting solenoid with a magnetic field of 3.5 T encases the calorimeters. An iron yoke outside the solenoid coil returns the magnetic flux and is instrumented with scintillator-based muon detectors. In the low angle region, charged particles will be efficiently tracked down to $7^{\circ}$. Dedicated calorimeters are placed in the forward region for detecting particles at even lower angles to the beam [44]. The most forward component of this system - the BeamCal-has holes for the beam pipe, which constitutes the only region outside detector acceptance, and corresponds to $5.6 \mathrm{mrad}$.

The simulation and reconstruction tools used in this study are part of the ILCSOFT framework (v01-16-02) [45]. The beam crossing angle of $14 \mathrm{mrad}$ and the response of the ILD detector in its version ILD_o1_v05 as used for the ILC TDR [28] are simulated using MOKKA [46] based on GEANT4. The event reconstruction is performed using the MARLIN [47] framework, including the particle flow algorithm PANDORAPFA [48] for calorimeter clustering and the analysis of track and calorimeter information.

\section{Signal processes and key observables}

We study the pair production of the two light charginos $\left(\tilde{\chi}_{1}^{+}, \tilde{\chi}_{1}^{-}\right)$and two light neutralinos $\left(\tilde{\chi}_{1}^{0}, \tilde{\chi}_{2}^{0}\right)$. In our benchmark models, the Higgsino component is strongly dominant for these four light states. Their masses are shown in Table I. The charginos and neutralinos are both produced dominantly via the $s$-channel exchange since the sleptons are assumed to be heavy. The chargino pair production proceeds as $e^{+} e^{-} \rightarrow \tilde{\chi}_{1}^{+} \tilde{\chi}_{1}^{-}$through the $\gamma / Z$ exchange, while the neutralino associated production $e^{+} e^{-} \rightarrow \tilde{\chi}_{1}^{0} \tilde{\chi}_{2}^{0}$ undergoes via the $Z$ boson exchange. While in a real analysis at ILC, more decay modes of the $\tilde{\chi}_{2}^{0}$ and the $\tilde{\chi}_{1}^{ \pm}$can be utilized, we focus here on the semileptonic channel for the charginos, i.e., $\tilde{\chi}_{1}^{+} \tilde{\chi}_{1}^{-} \rightarrow q \bar{q}^{\prime} \tilde{\chi}_{1}^{0} \ell \nu \tilde{\chi}_{1}^{0}$, and on the leptonic channel for the neutralinos, i.e., $\tilde{\chi}_{2}^{0} \tilde{\chi}_{1}^{0} \rightarrow \ell^{+} \ell^{-} \tilde{\chi}_{1}^{0}$. We restrict $\ell=e, \mu$ in this study.

The key target observables are the three masses $\left(M_{\tilde{\chi}_{1}^{0}}, M_{\tilde{\chi}_{2}^{0}}\right.$, and $\left.M_{\tilde{\chi}_{1}^{ \pm}}\right)$and, in this study, four polarized cross sections: chargino and neutralino production for the two opposite-sign beam polarization configurations. In a real ILC analysis, the like-sign combinations would be included as well, at least to serve as background-enriched control samples.

The three masses can be extracted from the maximum end points of the kinematic distributions shown at the generator level in Figs. 3(a), 3(b), 3(d), and 3(e). Specifically, we will rely on the maximum invariant mass and energy of the visible decay products of the $\tilde{\chi}_{2}^{0} \rightarrow \ell^{+} \ell^{-} \tilde{\chi}_{1}^{0}$ and $\tilde{\chi}_{1}^{ \pm} \rightarrow q \bar{q}^{\prime} \tilde{\chi}_{1}^{0}$. We find that the minimum end points, typically used in other SUSY studies, are too small to be useful in this study, since the resulting detector response is challenging to model in the soft spectrum, and in the case of the neutralino channel, it has large overlap with irreducible backgrounds.

The maximum energy $E_{\max }$ of the dijet (or dilepton) system seen in the laboratory frame is given $\mathrm{by}^{7}$

$$
E_{\max }=\frac{\gamma(1+\beta)}{2}\left(1+\frac{M}{M^{\prime}}\right) \Delta M,
$$

where $M$ is the $\operatorname{LSP}\left(\tilde{\chi}_{1}^{0}\right)$ mass and $M^{\prime}$ is the mass of $\tilde{\chi}_{1}^{ \pm}\left(\tilde{\chi}_{2}^{0}\right)$ for the chargino (neutralino) channel. The mass difference is given by $\Delta M=M^{\prime}-M$. As the decays studied are three body decays, it follows that the Lorentz-invariant mass of any pair of final-state particles has a maximum equal to the mass difference between the decaying particle and the mass of the third decay product; see, e.g., Sec. 47.4.4.1 of [49]. In other words, the maximum of the dijet (or dilepton) mass

\footnotetext{
${ }^{7}$ The value for $E_{\max }$ given in formula Eq. (2) is attained when the dilepton or dijet invariant mass is zero. The complete formulas relating the maximum and minimum energy to the invariant mass may be found on p. 439 of Ref. [13]. The sensitivity of the analysis could even be improved by evaluating the maximum energy on an event-by-event basis, taking into account the dijet / dilepton invariant mass measured in each event individually.
} 


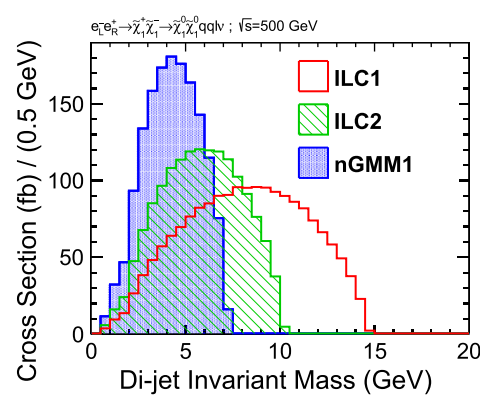

(a) chargino channel

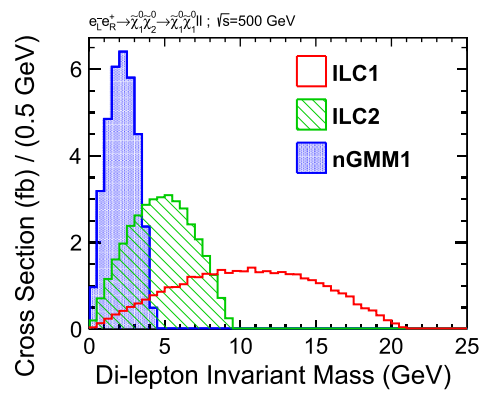

(d) neutralino channel

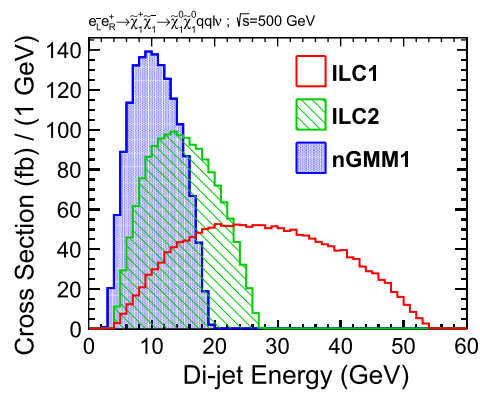

(b) chargino channel

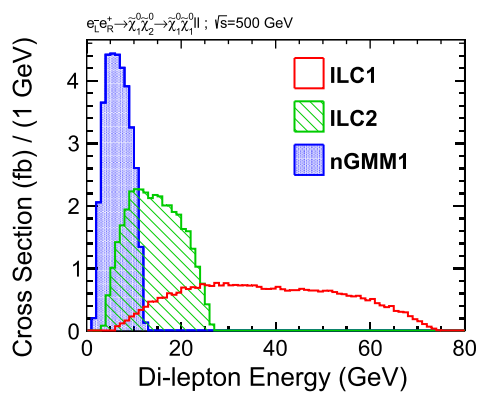

(e) neutralino channel

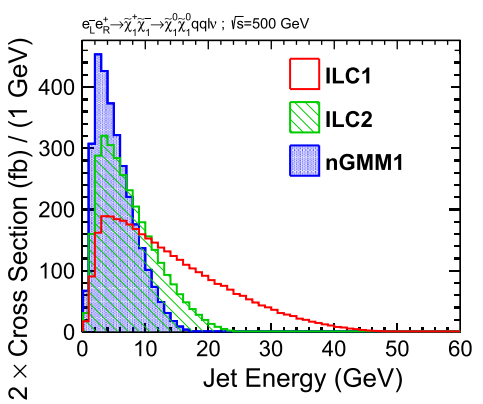

(c) chargino channel

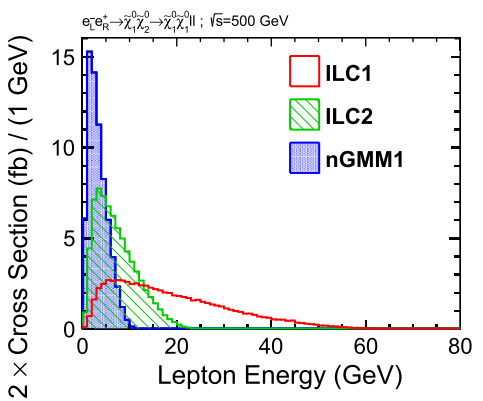

(f) neutralino channel

FIG. 3. Generator-level distributions, given for the beam polarization with $\mathcal{P}_{L R}$. The distributions for the other beam polarization $\mathcal{P}_{R L}$ are similar, up to the normalization due to the cross section.

is a direct measure of $\Delta M$. The boost factors $\beta$ and $\gamma$ are given according to $\gamma=\left(1-\beta^{2}\right)^{-\frac{1}{2}}$ and $\beta=\frac{p}{\sqrt{M^{\prime 2}+p^{2}}}$, where the maximum momentum $p$ in the laboratory frame is given by

$p=\frac{\sqrt{s}}{2} \sqrt{1-2\left[\left(\frac{M}{\sqrt{s}}\right)^{2}+\left(\frac{M^{\prime}}{\sqrt{s}}\right)^{2}\right]+\left[\left(\frac{M^{\prime}}{\sqrt{s}}\right)^{2}-\left(\frac{M}{\sqrt{s}}\right)^{2}\right]^{2}}$.

For any given channel, the measurements of $\Delta M$ and $E$ yield the masses $M$ and $M^{\prime}$ by numerically solving the relations above. In our study, we obtain several measurements of $\Delta M$ and $E$, specifically for the two different lepton final states, $(\ell=e$ and $\mu)$, and for the two different beam polarizations, $\mathcal{P}_{-+}$and $\mathcal{P}_{+-}$. These measurements can be readily combined individually for the chargino and neutralino channels. Because the chargino and neutralino measurements both include the LSP mass $M_{\tilde{\chi}_{1}^{0}}$ in the observables, a final combination is performed using a fit to extract the uncertainty of the three masses.

Figure 3 shows the generator-level distributions of the mass and energy distributions for the dijet (dilepton) system for the chargino (neutralino) channel. The three benchmark points, ILC1, ILC2, and nGMM1, give progressively softer distributions due to the smaller mass gaps. The visible part of the chargino/neutralino decay will be very soft; for example, most of the jets and leptons have energies less than $20 \mathrm{GeV}$ in the case of the ILC2 benchmark, and less than $10-15 \mathrm{GeV}$ in the case of the nGMM1 benchmark.

\section{Parameter fitting}

In the final step of the study, we investigate the possibility to extract SUSY parameters from the projected measurement precisions obtained from the detector simulation. This will be addressed using a Markov chain technique as implemented in the program FITTINO. Unless stated otherwise, the length of the Markov chains is $10^{6}$ for each fitted configuration. While the MC samples used in the full detector simulation were based on ISAJET [50], FITTINO employs SPHENO[51] as a spectrum calculator during the fit. More details about the fitting procedure can be found in [52].

In addition to the mass and cross section projections from this study, which will be described in detail in Sec. IV, a standard set of projected Higgs precision observables from the ILC was used to constrain the fit: These projections assume the $\mathrm{H} 20$ running scenario for the ILC [43] and include the Higgs mass (with a precision of $15 \mathrm{MeV}$ as obtained in a ILD full simulation study [53]), and a set of Higgs branching ratio precisions obtained from the model-independent coupling fit results in [54], based on the so-called $\kappa$ framework. ${ }^{8}$ The resulting precisions are shown in Fig. 4 in comparison to the expected deviations

\footnotetext{
${ }^{8}$ Note that this includes the cross section measurements, which in effect dominate the coupling precisions for the weak gauge bosons. For technical reasons, the coupling precisions could not be used directly in the fit.
} 


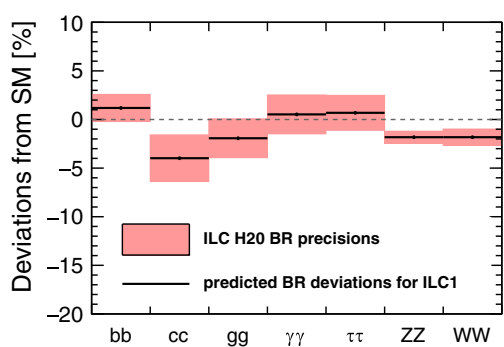

(a) ILC1

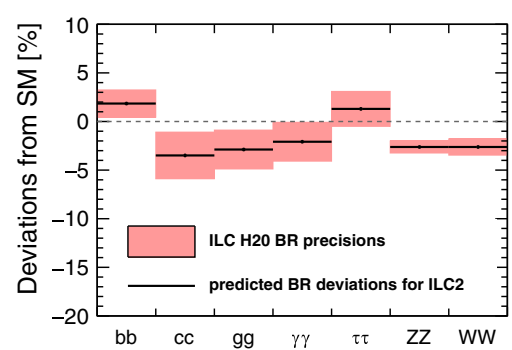

(b) ILC2

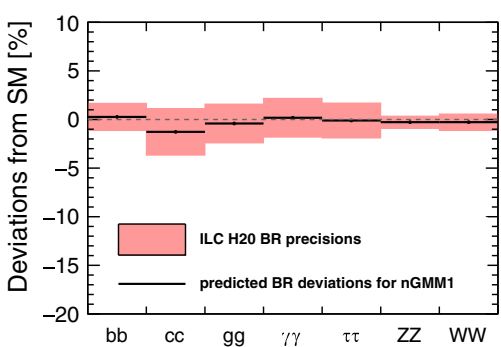

(c) nGMM1

FIG. 4. Deviations of the branching fractions of the SUSY light Higgs from the Standard Model expectations, as obtained with FEYNHIGGS2.10.4. For ILC1 and ILC2, the total width is about 2\%-3\% larger than its SM predicted value, which can enhance or suppress the effect of the Higgs-fermion coupling deviations on the branching fractions, depending on the sign of the coupling deviations. The uncertainty bands illustrate the expected measurement precisions after the full 250 and $500 \mathrm{GeV}$ ILC program, assuming the H20 scenario [54]. Note that these are somewhat more conservative than the most recent estimates from [56].

from the SM branching ratios in our three benchmarks. The Higgs mass and branching-ratio values are taken from FEYNHIGGS2.10.4 [55] for each of the SUSY models in question. For the NUHM2-based benchmarks, the most significant deviations would be observed in the Higgs couplings to the $W$ and $Z$ bosons, although they would be hardly convincing as a discovery on their own. In the case of nGMM1, all Higgs precision measurements agree perfectly with the SM.

\section{FULL DETECTOR SIMULATION STUDY}

For each benchmark point, we select separately the chargino and neutralino channels. These are considered as background to each other, including all decay modes. A common event selection is performed and provides sufficient sensitivity in all three benchmark cases. In a real experiment, once a signal has been discovered, the selection could be optimized based on initial mass estimates; see, e.g., [57] for a discussion of how to boot strap a selection of an a priori unknown signal. In the following sections, we describe the event selection for the chargino and neutralino channels.

\section{A. Chargino channel}

For the chargino pair production, we study the semileptonic final state $e^{-} e^{+} \rightarrow \tilde{\chi}_{1}^{+} \tilde{\chi}_{1}^{-} \rightarrow \tilde{\chi}_{1}^{0} \tilde{\chi}_{1}^{0} q q^{\prime} \ell \nu_{\ell}$, where $\ell=e$ or $\mu$. The strategy here is to look for a single isolated lepton accompanied by two jets and large missing energy. We reconstruct the invariant mass and energy distributions of the dijet system and extract their end points.

First, an isolated lepton candidate is identified according to the following criteria. Electron identification requires that the total energy measured in the calorimetric system $E_{\text {tot }}$ is consistent with the momentum measured in the tracker $p_{\text {trk }}$, such that they satisfy $0.5<E_{\text {tot }} / p_{\text {trk }}<1.3$. In addition, the energy deposit in the electromagnetic calorimeter $E_{\mathrm{ECAL}}$ must be dominant over the energy deposit in the hadronic calorimeter $E_{\mathrm{HCAL}}$, so that we have
$E_{\mathrm{ECAL}} /\left(E_{\mathrm{ECAL}}+E_{\mathrm{HCAL}}\right)>0.9$. For muon identification, we require that a charged track is associated with signals in the muon detector. In addition, lepton candidates with a large impact parameter significance $(>5 \sigma)$ are rejected in order to suppress backgrounds due to $\tau$ or heavy quark decays. For the isolation requirement, we define an isolation cone around the lepton candidate with a half-angle $\alpha$ such that $\cos \alpha=0.95$. We require that the total energy of charged particles within the cone (not including the candidate itself) is less than $0.2 \mathrm{GeV}$. The isolated lepton candidate with the highest transverse momentum is selected as the isolated lepton in the event.

Next, we deal with high cross section $\gamma \gamma$ processes that produce soft hadrons that overlap with our signal. Jet finders are used in two steps, following the procedures in [28]. We apply the $k_{t}$ jet finder algorithm with the jet radius parameter $R=1.4$, forcing all reconstructed particles of the event apart from the isolated lepton into two jets, plus two additional beam jets; particles that are clustered into the beam jets are removed in the remainder of the event reconstruction [58,59]. The value $R=1.4$ was chosen to yield dijet mass distributions, which are optimal for the extraction of the kinematic end point. The remaining particles are used to reconstruct the chargino that decayed hadronically. They are forced into two jets using the Durham jet finding algorithm [60].

The event selection proceeds as follows. We select events with exactly one isolated lepton candidate, and its lepton type is identified. We reject events containing particles that are reconstructed in the BeamCal [44]. The transverse momentum of the lepton is required to be $5 \mathrm{GeV}$ or greater. The number of reconstructed charged particles in each jet must be 2 or greater. It was tested whether a tighter cut on the track multiplicity would help to reject background from, e.g., three-prong $\tau$ decays, but due to low jet energies, especially in the ILC2 and nGMM1 benchmarks, cf. Fig. 3(c), the resulting loss in signal was too severe. Both of the reconstructed jets should not be very forward, so that the polar angle of each jet $\theta_{j}$ is such that $\left|\cos \theta_{j}\right|<0.98$. We require 
TABLE VI. Expected number of events after the event selection for the chargino signal and major backgrounds, normalized to an integrated luminosity of $500 \mathrm{fb}^{-1}$. For each benchmark model, the SUSY background is given in the column "Bkg.".

\begin{tabular}{|c|c|c|c|c|c|c|c|c|c|c|c|c|}
\hline \multicolumn{2}{|c|}{$e^{+} e^{-} \rightarrow \tilde{\chi}_{1}^{+} \tilde{\chi}_{1}^{-}$} & \multicolumn{2}{|c|}{ ILC1 } & \multicolumn{2}{|c|}{ ILC2 } & \multicolumn{2}{|c|}{ nGMM1 } & \multicolumn{5}{|c|}{ SM backgrounds } \\
\hline Process & $\mathcal{P}$ & Signal & Bkg. & Signal & Bkg. & Signal & Bkg. & $e^{+} e^{-} \rightarrow 2 \mathrm{f}$ & $e^{+} e^{-} \rightarrow 4 \mathrm{f}$ & $\gamma \gamma \rightarrow 2 \mathrm{f}$ & $e \gamma \rightarrow 3 \mathrm{f}$ & $\gamma \gamma \rightarrow 4 \mathrm{f}$ \\
\hline \multirow[t]{2}{*}{$q q e \nu$} & $\mathcal{P}_{-+}$ & 1463 & 85 & 392 & 23 & 283 & 15 & 5.9 & 64 & 0.0 & 22 & 2.0 \\
\hline & $\mathcal{P}_{+-}$ & 404 & 23 & 96 & 4.6 & 73 & 5.1 & 7.4 & 16 & 0.0 & 8.0 & 2.0 \\
\hline \multirow[t]{2}{*}{$q q \mu \nu$} & $\mathcal{P}_{-+}$ & 1862 & 108 & 509 & 28 & 389 & 29 & 33 & 37 & 0.0 & 0.0 & 7.0 \\
\hline & $\mathcal{P}_{+-}^{+}$ & 524 & 34 & 127 & 8.5 & 101 & 8.2 & 8.2 & 7.2 & 0.0 & 0.0 & 7.0 \\
\hline
\end{tabular}

the coplanarity of the two jets as defined by the difference of the azimuthal angle to be $\Delta \phi=\left|\phi_{2}-\phi_{1}\right|<1.0$. The angle between the two jets $\theta_{j j}$ is required to satisfy $\left|\cos \theta_{j j}\right|<0.2$. The visible energy in the event is required to be less than $80 \mathrm{GeV}$. The missing energy in the event is required to be greater than $400 \mathrm{GeV}$. The polar angle of the missing momentum $\theta_{\text {miss }}$ is required to satisfy $\left|\cos \theta_{\text {miss }}\right|<0.99$. The expected number of signal and background events after the event selection is shown in Table VI. Very few background events survive after the event selection. An example of the resulting distributions is shown for the $q q \mu \nu$ channel with the beam polarization $\mathcal{P}_{-+}$in Fig. 5. The number of events at various steps of the event selection and the distributions for all studied channels can be found in the Appendix.

The maximum end points of the dijet energy $\left(E_{j j}\right)$ and mass $\left(M_{j j}\right)$ distributions are extracted using a fit. Figures 6-7 show some examples of such a fit. Although

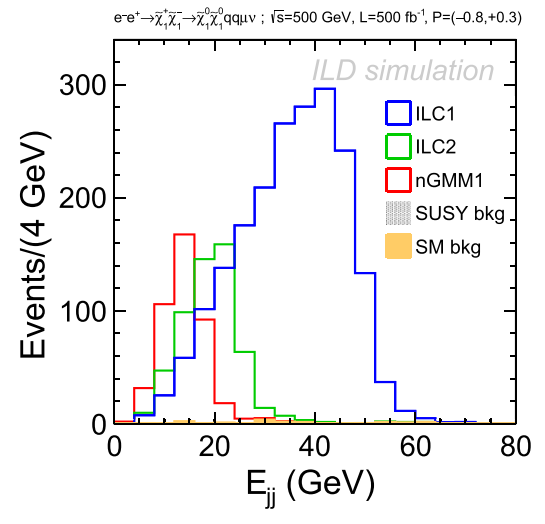

(a) di-jet energy

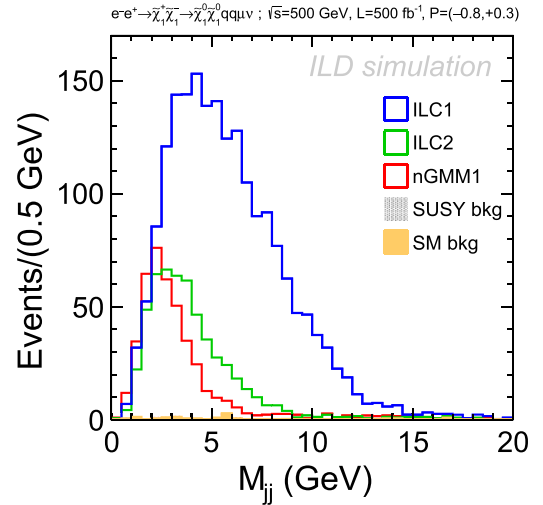

(b) di-jet mass

FIG. 5. Example of reconstructed distributions in the chargino channel $e^{+} e^{-} \rightarrow \tilde{\chi}_{1}^{+} \tilde{\chi}_{1}^{-} \rightarrow \tilde{\chi}_{1}^{0} \tilde{\chi}_{1}^{0} q \bar{q}^{\prime} \mu \nu_{\mu}$ with a beam polarization $\mathcal{P}_{-+}$. The contributions from SUSY and SM backgrounds are very small. The signal histograms are stackeed on top of the backgrounds.

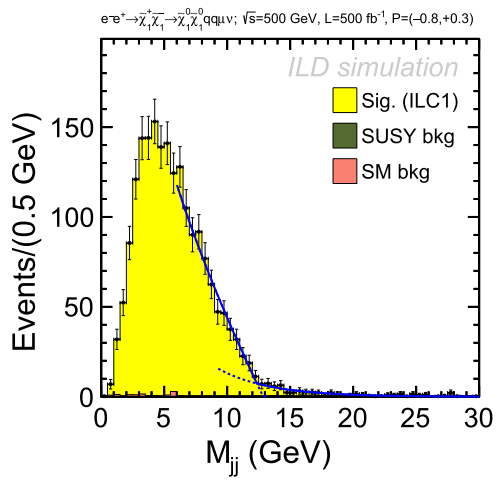

(a) ILC1

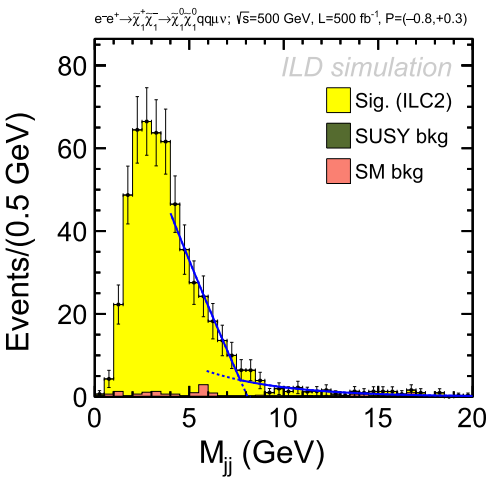

(b) ILC2

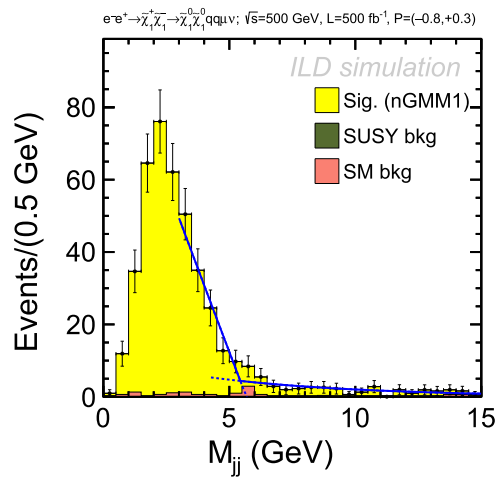

(c) nGMM1

FIG. 6. Example of the end point extraction of the dijet invariant mass system in the chargino channel $e^{-} e^{+} \rightarrow \tilde{\chi}_{1}^{+} \tilde{\chi}_{1}^{-} \rightarrow \tilde{\chi}_{1}^{0} \tilde{\chi}_{1}^{0} q \bar{q}^{\prime} \mu \nu_{\mu}$ with a beam polarization $\mathcal{P}_{-+}$for the three benchmarks. 


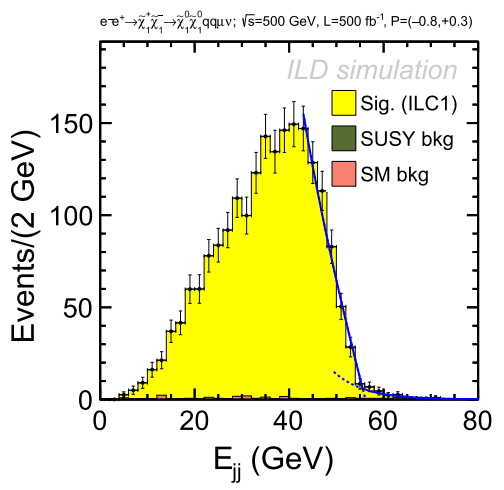

(a) ILC1

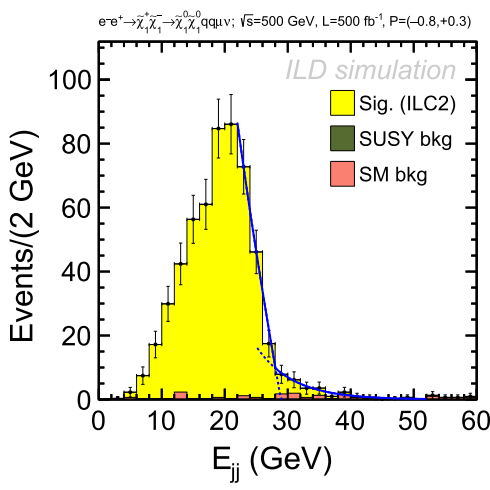

(b) ILC2

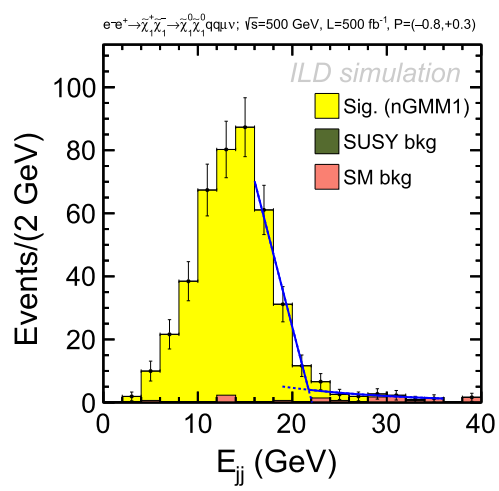

(c) nGMM1

FIG. 7. Example of the end point extraction of the dijet energy system in the chargino channel $e^{-} e^{+} \rightarrow \tilde{\chi}_{1}^{+} \tilde{\chi}_{1}^{-} \rightarrow \tilde{\chi}_{1}^{0} \tilde{\chi}_{1}^{0} q \bar{q}^{\prime} \mu \nu_{\mu}$ with a beam polarization $\mathcal{P}_{-+}$for the three benchmarks.

the samples are almost free of backgrounds, the signal distribution has a tail which is caused by the failure to properly reconstruct the energy of soft neutral particles. We use an exponential curve to model such an effect, combined with a linear function to model the steep drop leading to the kinematic end point. The point where the two functions meet after the fit was used to estimate the kinematic end point. The statistical uncertainty of this value is estimated by performing toy Monte Carlo experiments that repeatedly fit statistically fluctuated versions of these parent distributions. The extracted maximum end point of the dijet mass distributions is seen to have a systematic shift from the actual mass difference, which requires a correction at the level of 10\%-20\% before they are used input to the final fit for the masses. It is assumed here that such a calibration procedure does not add any significant systematic uncertainties to our results, as described in [61]. The end points from the dijet energy distributions are used without corrections in the mass fit.

\section{B. Neutralino channel}

For the neutralino mixed production, we choose the clean leptonic decay of $\tilde{\chi}_{2}^{0}$ as the final state: $e^{-} e^{+} \rightarrow$ $\tilde{\chi}_{1}^{0} \tilde{\chi}_{2}^{0} \rightarrow \tilde{\chi}_{1}^{0} \tilde{\chi}_{1}^{0} \ell^{+} \ell^{-}$, where $\ell=e$ or $\mu$. The strategy is to look for a pair of isolated leptons with a large missing energy. The invariant mass and the energy of the dilepton system provides information about the neutralino masses.
The isolated leptons are selected in the same way as in the chargino channel. This time, we require two oppositely charged leptons of the same flavor, instead of one.

The expected number of signal and major background events are summarized in Table VII; the full tables of event selection can be found in the Appendix. At the preselection stage, we require that two oppositely charged leptons are found, each having a transverse momentum of at least $2 \mathrm{GeV}$. Then, the lepton flavor is required to be either an electron or a muon pair, and the total number of reconstructed charged particles (including the leptons) in the event is required to be exactly two. We reject events containing particles that are reconstructed in the BeamCal. The requirement on the transverse momentum of both leptons are further tightened to $2.3 \mathrm{GeV}$ or greater. The polar angle of each lepton's momentum $\theta_{\ell}$ is required to satisfy $\left|\cos \theta_{\ell}\right|<0.95$. The coplanarity of the two leptons $\Delta \phi=\left|\phi_{1}-\phi_{2}\right|$ is required to satisfy $\Delta \phi<0.8$. The visible energy of the event is required to be less than $25 \mathrm{GeV}$. The missing energy in the event must be greater than $300 \mathrm{GeV}$. The polar angle of the missing momentum angle $\theta_{\text {miss }}$ must satisfy $\left|\cos \theta_{\text {miss }}\right|<0.98$.

An example of the $M_{\ell \ell}$ and $E_{\ell \ell}$ distributions after this selection is shown in Fig. 8; the full distributions can be found in the Appendix. In contrast to the chargino channel, the neutralino channel has sizable SM backgrounds after the event selection, since due to the much smaller number

TABLE VII. Expected number of events after the event selection for the neutralino signal and major backgrounds, normalized to an integrated luminosity of $500 \mathrm{fb}^{-1}$. For each benchmark model, the SUSY background is given in the column "Bkg.".

\begin{tabular}{|c|c|c|c|c|c|c|c|c|c|c|c|c|}
\hline \multicolumn{2}{|c|}{$e^{+} e^{-} \rightarrow \tilde{\chi}_{1}^{0} \tilde{\chi}_{2}^{0}$} & \multicolumn{2}{|c|}{ ILC1 } & \multicolumn{2}{|c|}{ ILC2 } & \multicolumn{2}{|c|}{ nGMM1 } & \multicolumn{5}{|c|}{ SM bkg. } \\
\hline Process & $\mathcal{P}$ & Sig. & Bkg. & Sig. & Bkg. & Sig. & Bkg. & $e^{+} e^{-} \rightarrow 2 \mathrm{f}$ & $e^{+} e^{-} \rightarrow 4 \mathrm{f}$ & $\gamma \gamma \rightarrow 2 \mathrm{f}$ & $e \gamma \rightarrow 3 \mathrm{f}$ & $\gamma \gamma \rightarrow 4 \mathrm{f}$ \\
\hline$e e$ & $\begin{array}{l}\mathcal{P}_{-+} \\
\mathcal{P}_{+-}\end{array}$ & $\begin{array}{l}1621 \\
1284\end{array}$ & $\begin{array}{r}185 \\
69\end{array}$ & $\begin{array}{l}1250 \\
1017\end{array}$ & $\begin{array}{l}226 \\
111\end{array}$ & $\begin{array}{l}490 \\
409\end{array}$ & $\begin{array}{l}207 \\
119\end{array}$ & $\begin{array}{l}14 \\
13\end{array}$ & $\begin{array}{r}3875 \\
508\end{array}$ & $\begin{array}{l}14 \\
14\end{array}$ & $\begin{array}{r}371 \\
83\end{array}$ & $\begin{array}{l}19 \\
19\end{array}$ \\
\hline$\mu \mu$ & $\begin{array}{l}\mathcal{P}_{-+} \\
\mathcal{P}_{+-}\end{array}$ & $\begin{array}{l}1939 \\
1521 \\
\end{array}$ & $\begin{array}{r}176 \\
49 \\
\end{array}$ & $\begin{array}{l}1496 \\
1222\end{array}$ & $\begin{array}{r}197 \\
67\end{array}$ & $\begin{array}{l}640 \\
516 \\
\end{array}$ & $\begin{array}{l}91 \\
40\end{array}$ & $\begin{array}{l}0.0 \\
0.0\end{array}$ & $\begin{array}{r}5506 \\
672\end{array}$ & $\begin{array}{l}77 \\
77 \\
\end{array}$ & $\begin{array}{l}100 \\
100\end{array}$ & $\begin{array}{l}9.6 \\
9.6\end{array}$ \\
\hline
\end{tabular}




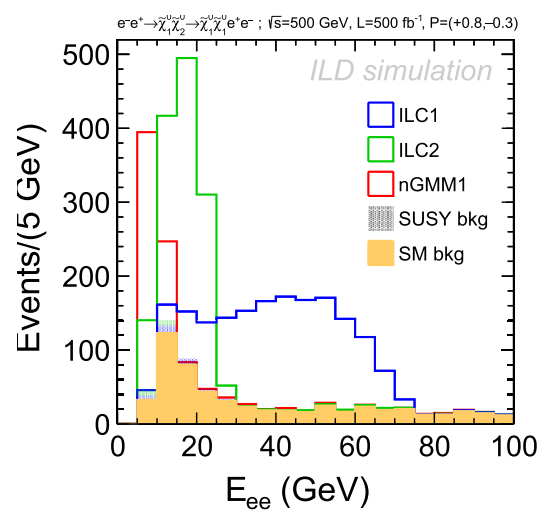

(a) di-jet energy

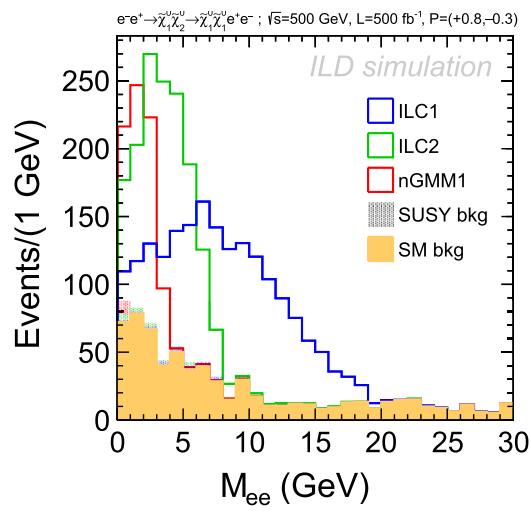

(b) di-jet mass

FIG. 8. Example of reconstructed distributions in the neutralino channel $e^{-} e^{+} \rightarrow \tilde{\chi}_{1}^{0} \tilde{\chi}_{2}^{0} \rightarrow \tilde{\chi}_{1}^{0} \tilde{\chi}_{1}^{0} e^{+} e^{-}$with a beam polarization $\mathcal{P}+$. The signal histograms are stacked on top of the backgrounds. The remaining background is fully dominated by the SM contribution.

of signal events the cuts cannot be as tight as in the chargino case. The dominant backgrounds are the $e^{+} e^{-} \rightarrow$ $\ell^{+} \ell^{-} \nu_{\ell^{\prime}} \bar{\nu}_{\ell^{\prime}}$ processes, where $\ell$ is the same lepton flavor as the final state leptons of the signal. The SUSY backgrounds remain negligible.

The maximum end points of the energy $\left(E_{\ell \ell}\right)$ and mass $\left(M_{\ell \ell}\right)$ distributions of the dilepton system are extracted using a fit. We show some examples of the fit in Figs. 9-10. We use an exponential curve to model the background near the end point. A linear function is used to model the signal part. The intersection of the two functions is used to extract the kinematic end point. Again, the uncertainty of this value is estimated using toy Monte Carlo experiments. Fitting the invariant mass distribution in the nGMM1 benchmark point requires special care due to the $J / \psi$ resonance from the neutralino decay, which sits on the falling end of the distribution. The fit is done in two steps. First, a Gaussian distribution with a narrow width is used to fit the narrow peak in the small window of the $J / \psi$ resonance. The fitted yield and width of the resonance are fixed in the second, overall fit, which extracts the maximum end point. As was the case for the chargino channel, the extracted maximum end point of the dilepton mass distributions requires a correction at the level of $10 \%-20 \%$ before they are used input to the final fit for the masses, while the end points from the dilepton energy distributions are used without corrections.

\section{Results from the full detector simulation study}

We present the combined result of the mass measurements in Table VIII. Assuming an integrated luminosity of $500 \mathrm{fb}^{-1}$ at each of the two beam polarizations $\mathcal{P}\left(e^{+}, e^{-}\right)=( \pm 30 \%, \mp 80 \%)$, it is shown that the chargino and neutralino masses can be measured to about $0.5 \%-$ $0.7 \%$ for benchmarks with mass gaps of $10 \mathrm{GeV}$ or larger, and better than $1 \%$ for benchmarks with mass gaps of a few GeV.

In the last column, the relative precisions on the masses have been scaled to the full luminosity foreseen to be collected at $\sqrt{s}=500 \mathrm{GeV}$ according to the $\mathrm{H} 20$ and $\mathrm{I} 20$ running scenarios of the ILC [43]. These values are considered to be conservative as they neglect further

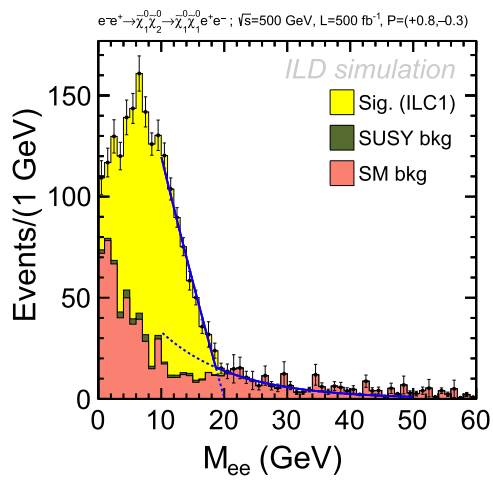

(a) ILC1

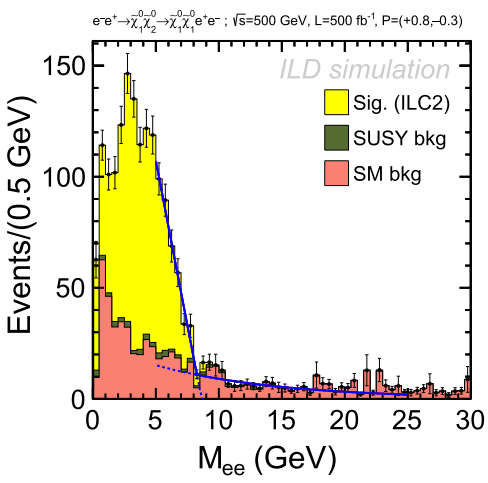

(b) ILC2

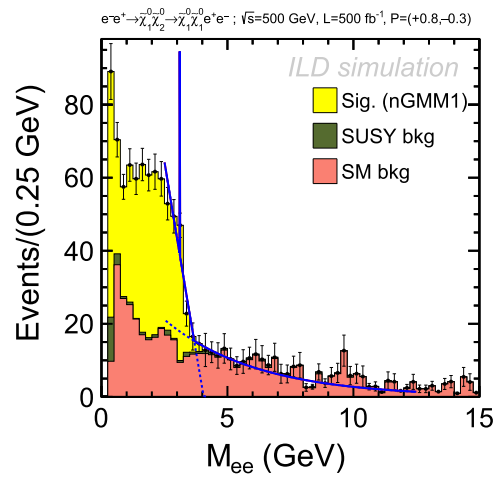

(c) nGMM1

FIG. 9. Example of the end point extraction of the dilepton invariant mass system in the neutralino channel $e^{-} e^{+} \rightarrow \tilde{\chi}_{1}^{0} \tilde{\chi}_{2}^{0} \rightarrow \tilde{\chi}_{1}^{0} \tilde{\chi}_{1}^{0} e^{+} e^{-}$ with a beam polarization $\mathcal{P}_{+-}$for the three benchmarks. For the nGMM1 benchmark, the $J / \psi$ peak is included in the fit. 


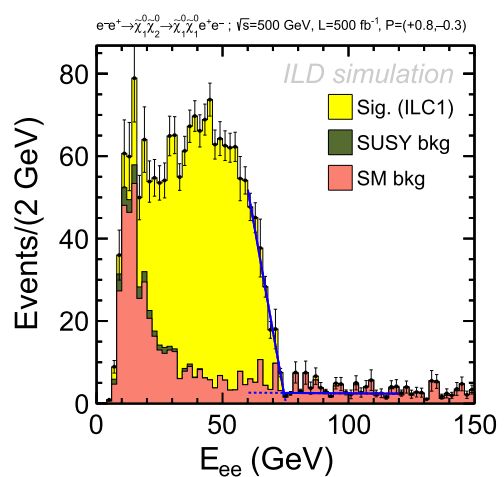

(a) ILC1

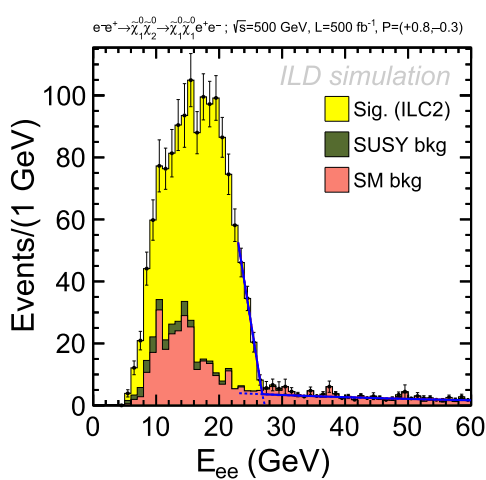

(b) ILC2

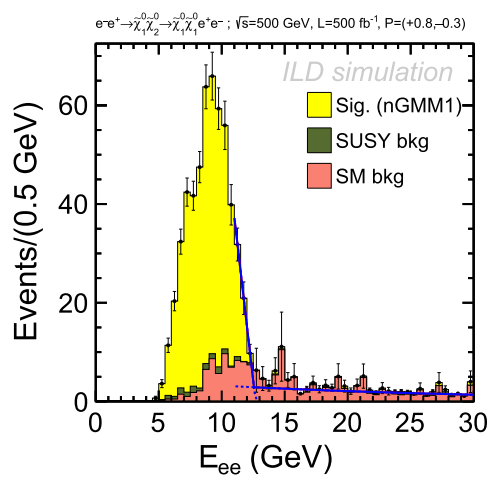

(c) nGMM1

FIG. 10. Example of the end point extraction of the dilepton energy system in the neutralino channel $e^{-} e^{+} \rightarrow \tilde{\chi}_{1}^{0} \tilde{\chi}_{2}^{0} \rightarrow \tilde{\chi}_{1}^{0} \tilde{\chi}_{1}^{0} e^{+} e^{-}$with a beam polarization $\mathcal{P}_{+-}$for the three benchmarks.

improvements from data sets at lower center-of-mass energies or from dedicated threshold scans. The relative precisions on the masses range from $0.3 \%$ in the case of ILC1 with the largest mass differences to about $0.6 \%$ for nGMM1 as in the case with the smallest mass differences.

The precision expected for the cross section times branching ratio measurements at $\sqrt{s}=500 \mathrm{GeV}$ is estimated from the statistical significance computed from the number of signal and background events using the dilepton and dijet energy distributions. In the case of the neutralino channel, an additional cut on the dilepton mass distribution is applied in order to remove the SM backgrounds in the high mass region; namely, events with $M_{\mathrm{M}_{\ell \ell}}$ less than 25, 15 , and $5 \mathrm{GeV}$ are selected for the ILC1, ILC2, and nGMM1 benchmarks, respectively. The accepted region

TABLE VIII. ILC1, ILC2, and nGMM1 MSSM model masses from ISAJET (see also Table I). Experimental mass precision from $\sqrt{s}=500 \mathrm{GeV}$ and $\mathcal{L}=500 \mathrm{fb}^{-1}$ combining both beam polarizations. It is assumed that the same precision is valid for these masses as the simulation shows for the ISAJET masses. The scaled precision for $1600 \mathrm{fb}^{-1}$ for each of the two opposite-sign polarization configurations at $\sqrt{s}=500 \mathrm{GeV}$, ignoring the data sets with other center-of-mass energies in the H2O and I20 operating scenarios.

\begin{tabular}{lcccc}
\hline \hline$\sqrt{s}=500 \mathrm{GeV}$ only & & ILC1 & ILC2 & nGMM1 \\
\hline Model mass [GeV] & $m_{\tilde{\chi}_{1}^{0}}$ & 102.7 & 148.1 & 151.4 \\
& $m_{\tilde{\chi}_{2}^{0}}$ & 124.0 & 157.8 & 155.8 \\
& $m_{\tilde{\chi}_{1}^{ \pm}}$ & 117.3 & 158.3 & 158.7 \\
Precision & $\delta m_{\tilde{\chi}_{1}^{0}} / m_{\tilde{\chi}_{1}^{0}}$ & $0.5 \%$ & $0.7 \%$ & $1.0 \%$ \\
$\left(\mathcal{P}_{-+}, \mathcal{L}=500 \mathrm{fb}^{-1}\right)$ & $\delta m_{\tilde{\chi}_{2}^{0}} / m_{\tilde{\chi}_{2}^{0}}$ & $0.5 \%$ & $0.7 \%$ & $1.0 \%$ \\
$\oplus\left(\mathcal{P}_{+-}, \mathcal{L}=500 \mathrm{fb}^{-1}\right)$ & $\delta m_{\tilde{\chi}_{1}^{ \pm}} / m_{\tilde{\chi}_{1}^{ \pm}}$ & $0.5 \%$ & $0.7 \%$ & $1.0 \%$ \\
Scaled precision & $\delta m_{\tilde{\chi}_{1}^{0}} / m_{\tilde{\chi}_{1}^{0}}$ & $0.3 \%$ & $0.4 \%$ & $0.5 \%$ \\
$\left(\mathcal{P}_{-+}, \mathcal{L}=1600 \mathrm{fb}^{-1}\right)$ & $\delta m_{\tilde{\chi}_{2}^{0}} / m_{\tilde{\chi}_{2}^{0}}$ & $0.3 \%$ & $0.4 \%$ & $0.5 \%$ \\
$\oplus\left(\mathcal{P}_{+-}, \mathcal{L}=1600 \mathrm{fb}^{-1}\right)$ & $\delta m_{\tilde{\chi}_{1}^{ \pm}} / m_{\tilde{\chi}_{1}^{ \pm}}$ & $0.3 \%$ & $0.4 \%$ & $0.5 \%$ \\
\hline \hline
\end{tabular}

of the energy distribution is optimized to yield the best statistical significance for each channel. These were extrapolated to lower center-of-mass energies based on cross section and luminosity scaling. In most cases, the cross sections can be measured with a precision of a few \%, as summarized in Table IX. Notable exceptions include the chargino cross sections for the $\mathcal{P}_{+-}$case, which are typically a factor of 2 worse than the other precisions.

\section{FITTING FUNDAMENTAL PARAMETERS}

In this section, we will pursue the question of whether the projected precisions on the physics observables will be sufficient to discriminate between different SUSY models and to determine the parameters of the correct model. To this purpose, assumed measurements of Higgsino masses and polarized cross sections are presented to FITTINO along with their projected uncertainties. It should be noted that the assumed measurements have not been varied randomly around their true values. Thus, in all cases where the correct underlying model is fitted, the expected $\chi^{2}$ is zero, apart from the effects of finite numerical precision and the finite length of the Markov chains. As discussed in Sec. III D, SPHENO had to be used instead of ISAJET as a spectrum calculator in the fitting step. ${ }^{9}$

The fits include the following inputs based on ILC simulation studies:

(i) The Higgsino masses obtained with SPHENO3.3.9BETA as listed in Table $\mathrm{X}$, together with their estimated precisions based on a preliminary version of the full simulation study at $\sqrt{s}=500 \mathrm{GeV}$, listed in Table 7.11 of Ref. [52]. Note that these are between $30 \%$ and $100 \%$ more conservative than

\footnotetext{
${ }^{9}$ Here, we switch to the spectrum generator SPHENO since there exists a direct interface between SPHENO and FITTINO, while no such interface exists for FITTINO and ISAJET. The mass spectra generated from ISAJET and SPHENO are slightly different due to different algorithms used by the code authors.
} 
TABLE IX. Estimated experimental precisions for the three benchmark points, for the four different final states, and the two beam polarizations. The full simulation results, performed for $\sqrt{s}=500 \mathrm{GeV}$, are given for $\mathcal{L}=500 \mathrm{fb}^{-1}$. The scaled precisions for the various center-of-mass energies are shown assuming the H20 scenario for ILC1 and the I20 scenario for ILC2 and nGMM1.

\begin{tabular}{|c|c|c|c|c|c|c|c|c|}
\hline \multirow[b]{2}{*}{$\Delta(\sigma \times \mathrm{BR})[\%]$} & \multicolumn{2}{|c|}{$\sqrt{s}=500 \mathrm{GeV}$} & \multicolumn{2}{|c|}{$\sqrt{s}=500 \mathrm{GeV}$} & \multicolumn{2}{|c|}{$\sqrt{s}=250 \mathrm{GeV}$} & \multicolumn{2}{|c|}{$\sqrt{s}=350 \mathrm{GeV}$} \\
\hline & $\mathcal{P}_{-+}$ & $\mathcal{P}_{+-}$ & $\mathcal{P}_{-+}$ & $\mathcal{P}_{+-}$ & $\mathcal{P}_{-+}$ & $\mathcal{P}_{+-}$ & $\mathcal{P}_{-+}$ & $\mathcal{P}_{+-}$ \\
\hline ILC1 & \multicolumn{2}{|c|}{$\mathcal{L}=500 \mathrm{fb}^{-1}$} & \multicolumn{6}{|c|}{ Scaled (H20) } \\
\hline$\tilde{\chi}_{1}^{0} \tilde{\chi}_{2}^{0} \rightarrow \tilde{\chi}_{1}^{0} \tilde{\chi}_{1}^{0} e e$ & 3.98 & 3.13 & 2.22 & 1.75 & 2.04 & 1.60 & 6.94 & 5.47 \\
\hline$\tilde{\chi}_{1}^{0} \tilde{\chi}_{2}^{0} \rightarrow \tilde{\chi}_{1}^{0} \tilde{\chi}_{1}^{0} \mu \mu$ & 3.81 & 2.97 & 2.13 & 1.66 & 1.95 & 1.52 & 6.66 & 5.18 \\
\hline$\tilde{\chi}_{1}^{+} \tilde{\chi}_{1}^{-} \rightarrow \tilde{\chi}_{1}^{0} \tilde{\chi}_{1}^{0} q q e \nu_{e}$ & 2.59 & 4.94 & 1.45 & 2.76 & 1.61 & 3.22 & 4.66 & 9.04 \\
\hline$\tilde{\chi}_{1}^{+} \tilde{\chi}_{1}^{-} \rightarrow \tilde{\chi}_{1}^{0} \tilde{\chi}_{1}^{0} q q \mu \nu_{\mu}$ & 2.27 & 4.30 & 1.27 & 2.40 & 1.41 & 2.80 & 4.09 & 7.87 \\
\hline ILC2 & \multicolumn{2}{|c|}{$\mathcal{L}=500 \mathrm{fb}^{-1}$} & \multicolumn{6}{|c|}{ Scaled (I20) } \\
\hline$\tilde{\chi}_{1}^{0} \tilde{\chi}_{2}^{0} \rightarrow \tilde{\chi}_{1}^{0} \tilde{\chi}_{1}^{0} e e$ & 3.92 & 3.50 & 2.19 & 1.96 & $\cdots$ & $\cdots$ & 2.82 & 2.52 \\
\hline$\tilde{\chi}_{1}^{0} \tilde{\chi}_{2}^{0} \rightarrow \tilde{\chi}_{1}^{0} \tilde{\chi}_{1}^{0} \mu \mu$ & 3.90 & 3.33 & 2.18 & 1.86 & $\ldots$ & $\ldots$ & 2.81 & 2.40 \\
\hline$\tilde{\chi}_{1}^{+} \tilde{\chi}_{1}^{-} \rightarrow \tilde{\chi}_{1}^{0} \tilde{\chi}_{1}^{0} q q e \nu_{e}$ & 5.17 & 10.30 & 2.89 & 5.76 & $\ldots$ & $\ldots$ & 4.09 & 8.28 \\
\hline$\tilde{\chi}_{1}^{+} \tilde{\chi}_{1}^{-} \rightarrow \tilde{\chi}_{1}^{0} \tilde{\chi}_{1}^{0} q q \mu \nu_{\mu}$ & 4.39 & 8.84 & 2.45 & 4.94 & $\cdots$ & $\cdots$ & 3.47 & 7.10 \\
\hline nGMM1 & \multicolumn{2}{|c|}{$\mathcal{L}=500 \mathrm{fb}^{-1}$} & \multicolumn{6}{|c|}{ Scaled (I20) } \\
\hline$\tilde{\chi}_{1}^{0} \tilde{\chi}_{2}^{0} \rightarrow \tilde{\chi}_{1}^{0} \tilde{\chi}_{1}^{0} e e$ & 5.30 & 4.98 & 2.96 & 2.78 & $\ldots$ & $\ldots$ & 3.84 & 3.61 \\
\hline$\tilde{\chi}_{1}^{0} \tilde{\chi}_{2}^{0} \rightarrow \tilde{\chi}_{1}^{0} \tilde{\chi}_{1}^{0} \mu \mu$ & 5.05 & 4.64 & 2.82 & 2.59 & $\ldots$ & $\cdots$ & 3.66 & 3.36 \\
\hline$\tilde{\chi}_{1}^{+} \tilde{\chi}_{1}^{-} \rightarrow \tilde{\chi}_{1}^{0} \tilde{\chi}_{1}^{0} q q e \nu_{e}$ & 6.20 & 11.73 & 3.47 & 6.56 & $\cdots$ & $\cdots$ & 4.94 & 9.48 \\
\hline$\tilde{\chi}_{1}^{+} \tilde{\chi}_{1}^{-} \rightarrow \tilde{\chi}_{1}^{0} \tilde{\chi}_{1}^{0} q q \mu \nu_{\mu}$ & 4.99 & 9.90 & 2.79 & 5.53 & $\ldots$ & $\ldots$ & 3.98 & 8.00 \\
\hline
\end{tabular}

TABLE X. ILC1, ILC2, and nGMM1 MSSM model masses from SPHENO3.3.9BETA together with the input precisions assumed in the fit. The assumed input precisions are given for $\sqrt{s}=$ $500 \mathrm{GeV}$ and $\mathcal{L}=1600 \mathrm{fb}^{-1}$. Data sets with other center-ofmass energies in the $\mathrm{H} 20$ and $\mathrm{I} 20$ operating scenarios are neglected.

\begin{tabular}{lrrrc}
\hline \hline$\sqrt{s}=500 \mathrm{GeV}$ only & & ILC1 & ILC2 & nGMM1 \\
\hline Model mass [GeV] & $m_{\tilde{\chi}_{1}^{0}}$ & 104.8 & 151.3 & 154.9 \\
& $m_{\tilde{\chi}_{2}^{0}}$ & 127.5 & 162.4 & 160.2 \\
& $m_{\tilde{\chi}_{1}^{ \pm}}$ & 116.0 & 157.0 & 157.4 \\
Assumed precision & $\delta m_{\tilde{\chi}_{1}^{0}} / m_{\tilde{\chi}_{1}^{0}}$ & $0.5 \%$ & $0.7 \%$ & $1.0 \%$ \\
$\left(\mathcal{P}_{-+}, \mathcal{L}=1600 \mathrm{fb}^{-1}\right)$ & $\delta m_{\tilde{\chi}_{2}^{0}} / m_{\tilde{\chi}_{2}^{0}}$ & $0.4 \%$ & $0.7 \%$ & $1.0 \%$ \\
$\oplus\left(\mathcal{P}_{+-}, \mathcal{L}=1600 \mathrm{fb}^{-1}\right)$ & $\delta m_{\tilde{\chi}_{1}^{ \pm}} / m_{\tilde{\chi}_{1}^{ \pm}}$ & $0.5 \%$ & $0.7 \%$ & $1.0 \%$ \\
\hline \hline
\end{tabular}

the results given in Table VIII of this paper. We will study and discuss the relevance of these differences in Sec. VI D. At tree level, the Higgsino masses depend on $\mu, M_{1}, M_{2}$, and $\tan \beta$, and thus, precision measurements will directly constrain these parameters. At the one-loop level, also the various sfermion masses enter the calculation, e.g., via fermion-sfermion loops, which occur via the gauge couplings and are included in both SPHENO and ISAJET. ${ }^{10}$

\footnotetext{
${ }^{10}$ The full one-loop calculations can be found in Appendix D.3 of Ref. [62]. Therefore, some limited sensitivity to the sfermion mass parameters can be expected if the Higgsino masses are measured to sufficient precision [63].
}

(ii) The polarized total cross sections for chargino and neutralino production at all relevant center-of-mass energies with precisions as given in Tables 7.127.14 of Ref. [52]. As for the masses, these are between $20 \%$ and $100 \%$ more conservative than the full simulation results listed in Table IX, and we will study and discuss the relevance of these differences in Sec. VID. Note that in ILC1, Higgsino production is kinematically accessible at center-of-mass energies as low as $250 \mathrm{GeV}$, while for ILC2 and nGMM $1 \sqrt{s}=350 \mathrm{GeV}$ is the lowest ILC energy stage which allows Higgsino production. Therefore, we consider the alternative running scenario I20 [43] for these benchmarks. Since the cross sections depend mainly on the Higgsino masses and on their mixing nature, i.e., their mass differences, they will be sensitive to the same parameters as the Higgsino masses.

(iii) The mass of the lightest $C P$-even Higgs boson, with the ILC precision according to [53], as discussed in Sec. III D. The Higgs boson mass will be instrumental in order to constrain the combination of the trilinear coupling $A_{0}$ and the sfermion mass parameter $M_{0}$ (or $A_{t}$ and the stop mass parameter $M_{U_{3}}$ in case of the weak scale fits). Also, in models with RG running, large $A$ terms affect the running of gaugino masses and gaugino mass unification. ${ }^{11}$

\footnotetext{
${ }^{11}$ In Ref. [64], it is advocated that the extraction of running gaugino masses to high precision at the ILC may allow a window into large trilinear soft terms.
} 
(iv) The branching ratios $\mathrm{BR}(h \rightarrow b \bar{b}), \mathrm{BR}(h \rightarrow c \bar{c})$, $\mathrm{BR}\left(h \rightarrow \tau^{+} \tau^{-}\right), \quad \mathrm{BR}(h \rightarrow g g), \quad \mathrm{BR}\left(h \rightarrow W^{+} W^{-}\right)$, $\mathrm{BR}(h \rightarrow Z Z)$, and $\mathrm{BR}(h \rightarrow \gamma \gamma)$, with the ILC precisions according to [43], as discussed in Sec. III D. Since the Higgs branching ratios in our benchmarks exhibit only small deviations from the SM case, they are expected to constrain the sfermion masses as well an $m_{A}$ from below.

In total, the input comprises 25 observables.

In the following, we will start by discussing fits of different GUT-scale models in Sec. VA and before proceeding to the determination of weak-scale parameters in Sec. V B. Finally, we will address the predictions of the LSP's relic density in Sec. V C.

\section{A. Fitting GUT-scale parameters}

The fitting of GUT-scale parameters requires strong assumptions on the underlying SUSY breaking scheme. Since our benchmarks cover two very different approaches to unification, it is interesting to study whether these can be distinguished by directly fitting different GUT-scale models.

\section{Results of fitting NUHM2}

In the case of NUHM2, we fit the parameters $M_{1 / 2}, \mu$, $\tan \beta, M_{0}, A_{0}$, and $m_{A}$ to the observables described in Sec. IV C.

Table XI shows the best fit point and its 1 and $2 \sigma$ confidence intervals obtained in the case of the ILC1 benchmark, in comparison to the input model parameters. Tables XII and XIII give the analogous information for the ILC2 and nGMM1 benchmarks, respectively. In case of the ILC1 and ILC2 benchmarks, where NUHM2 is the correct underlying model, the $\chi^{2}$ of the best fit point is very small, and all fitted parameters agree well with their true input values. The $1 \sigma$ uncertainties for $M_{1 / 2}, \mu, \tan \beta$ are typically $10 \%$ or better, while $M_{0}, A_{0}$, and $m_{A}$, which enter only at loop level into the considered observables, are still determined within about $20 \%$.

TABLE XI. Fitted parameters in the fit of NUHM2 parameters to ILC1 observables in the $\mathrm{H} 20$ scenario. All values in $\mathrm{GeV}$ apart from $\tan \beta$. Note that the $\chi^{2}$ value of the model point is increased from 0 by the rounding errors of the observables in the inputs.

Parameter ILC1 NUHM2 true Best fit point $1 \sigma \mathrm{CL} 2 \sigma \mathrm{CL}$

\begin{tabular}{lcccc}
\hline$M_{1 / 2}$ & 568.3 & 556.7 & +24.3 & +37.7 \\
$\mu$ & 115.0 & 105.3 & -20.3 & -43.1 \\
$\tan \beta$ & 10.0 & 11.4 & -8.8 & +14.0 \\
$m_{A}$ & 1000 & 968 & -1.6 & +11.4 \\
$M_{0}$ & 7025 & 7685 & -167 & +1.6 \\
$A_{0}$ & -10427 & -1243 & -130 \\
$\chi^{2}$ & 0.0013 & 0.0011 & & +1917 \\
\hline
\end{tabular}

TABLE XII. Best fit point and confidence intervals of the NUHM2 parameters fitted to ILC2 SUSY and Higgs measurements in the I20 operating scenario. Note that the $\chi^{2}$ value of the model point is increased from 0 by the rounding errors of the observables in the inputs.

\begin{tabular}{|c|c|c|c|c|}
\hline Parameter & ILC2 NUHM2 true & Best fit point & $1 \sigma \mathrm{CL}$ & $2 \sigma \mathrm{CL}$ \\
\hline$M_{1 / 2}$ & 1200 & 1194 & $\begin{array}{l}+107 \\
-68\end{array}$ & $\begin{array}{l}+164 \\
-129\end{array}$ \\
\hline$\mu$ & 150.0 & 150.7 & $\begin{array}{l}+4.3 \\
-4.5\end{array}$ & $\begin{array}{l}+7.2 \\
-5.2\end{array}$ \\
\hline $\tan \beta$ & 15.0 & 16.0 & $\begin{array}{l}-4.5 \\
+26.2 \\
-6.6\end{array}$ & $\begin{array}{l}-3.2 \\
+28.8 \\
-6.6\end{array}$ \\
\hline$m_{A}$ & 1000 & 1008 & $\begin{array}{l}+141 \\
+118\end{array}$ & +256 \\
\hline$M_{0}$ & 5000 & 4788 & $\begin{array}{l}+2546 \\
+3137\end{array}$ & $\begin{array}{r}-3566 \\
+3283\end{array}$ \\
\hline$A_{0}$ & -8000 & -7663 & $\begin{array}{r}+3817 \\
+3926\end{array}$ & $\begin{array}{l}+3817 \\
+5342\end{array}$ \\
\hline$\chi^{2}$ & 0.0007 & 0.02848 & & \\
\hline
\end{tabular}

TABLE XIII. Best fit point and confidence intervals of the NUHM2 parameters fitted to nGMM1 SUSY and Higgs measurements in the I20 operating scenario.

\begin{tabular}{lccc}
\hline \hline Parameter & Best fit point & $1 \sigma \mathrm{CL}$ & $2 \sigma \mathrm{CL}$ \\
\hline$M_{1 / 2}$ & 2407 & +150 & +356 \\
$\mu$ & 155.6 & -135 & -215 \\
$\tan \beta$ & 10.0 & -1.9 & +4.0 \\
$m_{A}$ & 1603 & +2.1 & -2.9 \\
$M_{0}$ & -0.5 & -0.4 \\
$A_{0}$ & 3422 & -279 & +1026 \\
$\chi^{2}$ & -7409 & -8309 & -469 \\
\hline \hline
\end{tabular}

In the case of the nGMM1 benchmark, the $\chi^{2}$ of the best fit point is somewhat larger than for the other two benchmarks, but still so small that an NUHM2 interpretation of this benchmark cannot be rejected. This is not surprising as it has been constructed to have the physical observables very similar to ILC2. However, the best fit point is found for $M_{1 / 2}$ about a factor of 2 bigger, and $M_{A}$ about $60 \%$ larger, than in ILC2. This implies that a direct observation of the heavy Higgs bosons and the electroweakinos could distinguish the two models. However, this also raises the question of whether the weak-scale fits based on input from the Higgsino properties alone will be able to identify the nGMM1 benchmark unambiguously as a non-NUHM2 model, with a completely different underlying SUSY breaking mechanism. We will investigate this in the next section.

Based on the fitted NUHM2 parameters and their uncertainties, the mass spectrum of the unobserved sparticles can be predicted for all three benchmark cases. This is illustrated in Fig. 11. In the cases of ILC1 and ILC2, clear predictions for the masses of the electroweakinos and the heavy Higgs bosons are obtained in excellent agreement with the true model masses, providing motivation and an energy scale for further upgrades of the ILC. Due to the 


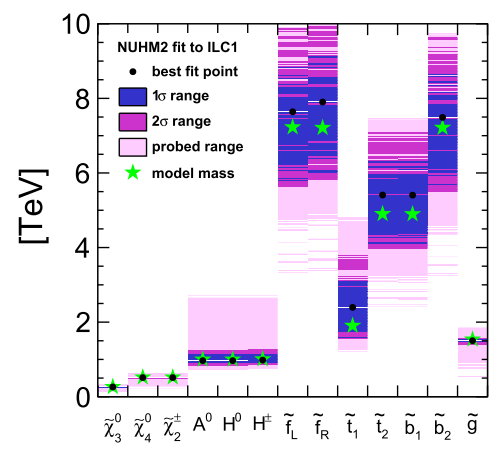

(a) ILC1

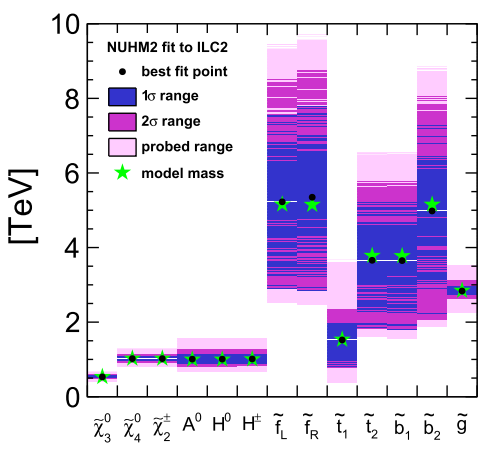

(b) ILC2

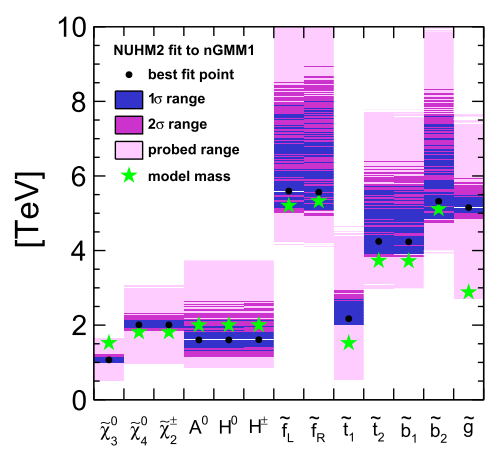

(c) nGMM1

FIG. 11. Predicted mass ranges for all the unobserved sparticles from the NUHM2 fit the observables of the three benchmark models. The green star indicates the true model mass, while the black dot shows the best fit point.

modeling of all gauginos by a single $M_{1 / 2}$ parameter, the gluino mass can also be firmly predicted, which can give important inputs to LHC analyses. If the predicted gluino were not observed, or observed at a very different mass, this would give strong support to the idea that nature does not describe all gauginos by one mass parameter at the GUT scale. The other sfermions are less well constrained than the Higgs bosons and gauginos, but upper limits on their masses can still be obtained. Such information would provide important motivation-and a target energy scalefor a future hadron collider.

Even in case of the nGMM1 benchmark, the NUHM2 fit predicts the general pattern of the mass spectrum correctly, albeit with less precision and significantly worse agreement between true model masses and the best fit point. The worse agreement is not surprising as we are fitting a wrong model hypothesis in this case. Nonetheless, upper limits on all sparticle masses are obtained, which shows that even in the case that the wrong model is assumed, such important information for the planning of future colliders or upgrades can be obtained.

\section{Results of fitting NUHM1 and CMSSM}

Before turning to the weak-scale fits, we investigate whether the three benchmarks could also be described by other widely used constrained models, in particular NUHM1 [65] and the CMSSM, which have one or even two fewer parameters to model the Higgs and Higgsino sectors: in NUHM1, instead of $M_{A}$ and $\mu$ (or $M_{H_{u}}^{0}$ and $M_{H_{d}}^{0}$ ), only one parameter, $M_{H}^{0}$, is used to describe the Higgs and Higgsino sectors, while in the CMSSM this reduces further to only the sign of $\mu$ being a free choice, while its absolute value is derived from the other model parameters. Table XIV gives the best fit point obtained when fitting NUHM1 and CMSSM to the ILC1 and ILC2 benchmarks, which give very large values of $\chi^{2} /$ d.o.f. These interpretations could be ruled out at the 95\% C.L. already with about $0.1 \%$ the total integrated luminosity.

\section{B. Weak scale fit results}

In the following, the results of various weak scale fits to the ILC1, ILC2, and nGMM1 observables are discussed. The most general model considered is pMSSM-10, the MSSM with ten weak scale input parameters: $M_{1}, M_{2}, M_{3}$, $\mu, \tan \beta, m_{A}, A_{t}=A_{b}=A_{\tau}$, and $M_{Q_{3}}, M_{U_{3}}=M_{D_{3}}$, $M_{L}=M_{L_{1,2,3}}=M_{E_{1,2,3}}=M_{Q_{1,2}}=M_{U_{1,2}}=M_{D_{1,2}}$. We use this model to test whether it is possible to constrain a comprehensive set of parameters from the observables of the Higgsino sector alone and to study the influence of the parameters in which the Higgsino sector enters only at loop level. If the pMSSM-10 fit is successful and reproduces the input parameters at a satisfactory level, we proceed to investigate the precision achievable when fitting only treelevel Higgsino parameters. For this, we use the phenomenological MSSM with a reduced number of four free parameters, the pMSSM-4, which fixes the squark, slepton, heavy Higgs boson, and gluino parameters to their true values and so only depends on the four weak scale parameters $M_{1,2}, \mu$, and $\tan \beta$. In a real analysis, the "true" values are of course unknown, but instead, the best fit point of pMSSM-10 fit could be used, which agrees with the model point to usually much better than $1 \sigma$. The possible

TABLE XIV. Best fit points in CMSSM and NUHM1 fits of ILC1 and ILC2 observables, including SUSY and Higgs measurements in the H20 operation for ILC1 and I20 operation for ILC2.

\begin{tabular}{lcccc}
\hline \hline $\begin{array}{l}\text { Best fit points } \\
\text { parameter }\end{array}$ & $\begin{array}{c}\text { ILC1 } \\
\text { CMSSM }\end{array}$ & $\begin{array}{c}\text { ILC2 } \\
\text { CMSSM }\end{array}$ & $\begin{array}{c}\text { ILC1 } \\
\text { NUHM1 }\end{array}$ & $\begin{array}{c}\text { ILC2 } \\
\text { NUHM1 }\end{array}$ \\
\hline$M_{1 / 2}[\mathrm{GeV}]$ & 128.8 & 155.9 & 136.9 & 159.9 \\
$M_{0}[\mathrm{GeV}]$ & 3585 & 5631 & 1796 & 4264 \\
$A_{0}[\mathrm{GeV}]$ & -6873 & -10873 & -4396 & -10086 \\
$\tan \beta$ & 13.7 & 16.2 & 16.2 & 14.8 \\
$M_{H_{0}}^{0}[\mathrm{GeV}]$ & $\cdots$ & $\cdots$ & 47659 & 56553 \\
$\chi_{\min }^{2}$ & 238046 & 93155 & 235014 & 85174 \\
$\chi^{2} /$ dof & 11336 & 4436 & 11751 & 4259 \\
\hline \hline
\end{tabular}


bias from fixing the six non-Higgsino parameters was tested explicitly in case of ILC1 by fixing them to some point in the $2 \sigma$ region. The best fit gaugino masses are nearly the same in the two pMSSM-4 fits irrespective of the fixed parameters. Their difference is only $2.5 \mathrm{GeV}$ for $\tilde{\chi}_{3}^{0}$ and $0.8 \mathrm{GeV}$ for $\tilde{\chi}_{4}^{0}$ and $\tilde{\chi}_{2}^{ \pm}$. The two sets of best fit masses agree within the $1 \sigma$ uncertainties [52]. In the following, the results for the individual benchmarks will be presented.

\section{ILC1 benchmark}

Figure 12 shows the minimum $\chi^{2}$ as a function of $M_{1}$, $M_{2}$, and $\mu$ and $\tan \beta$ in the pMSSM- 10 and pMSSM- 4 fits. Due to the much smaller parameter space to be sampled in the case of the four-dimensional fit, the resulting curve is much smoother than in the ten-dimensional case. The precision on $M_{1}$ is nearly identical in both cases. $M_{2}$ is somewhat better constrained in the pMSSM-4 fit, while the determination of $\mu$ and $\tan \beta$ improves drastically.

The resulting best fit values for the pMSSM parameters and their 1 and $2 \sigma$ intervals are compared to the input values in Table $\mathrm{XV}$, quantifying the effect which could already be seen qualitatively in Fig. 12. In the case of the pMSSM-10 fit, it should be noted that also for the parameters of the colored sector some constraints, and especially upper bounds, can be obtained. This even applies for the squark mass parameters, which might seem surprising at the first glance, but is due to the two-loop RGEs included in SPHENO. If a hypothetical gluino mass measurement with $11 \%$ uncertainty from the LHC [25] is included in the fit, the constraint on $M_{3}$ improves accordingly to about $10 \%$. All other parameters, including the squark mass parameters, show only minor improvements.

Figures 13(a) and 13(b) illustrate the precisions obtained on the pMSSM-10 parameters, without and with assuming a gluino mass measurement from the LHC, respectively. Thereby, $\tan \beta$ is displayed as if it were in $\mathrm{GeV}$. It can clearly be seen that the precision on $M_{3}$ is improved considerably by the gluino mass measurement, while the precision on all the other parameters do not change significantly.

The determined parameters can be used to predict the masses of the yet unobserved sparticles, as shown for the pMSSM-10 fit in Table XVI and Fig. 14, again without and

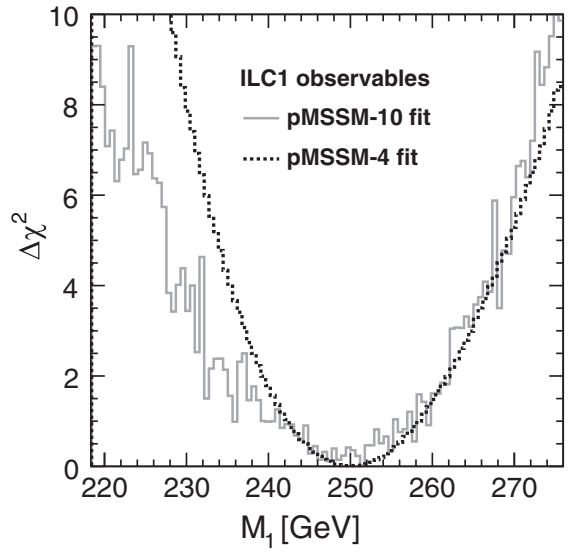

(a)

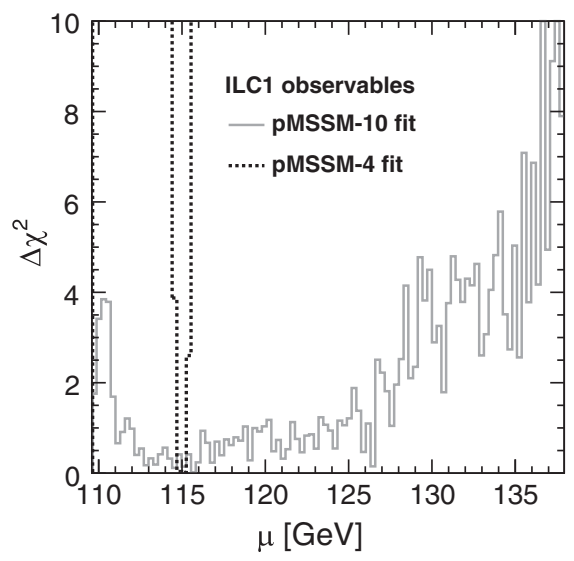

(c)

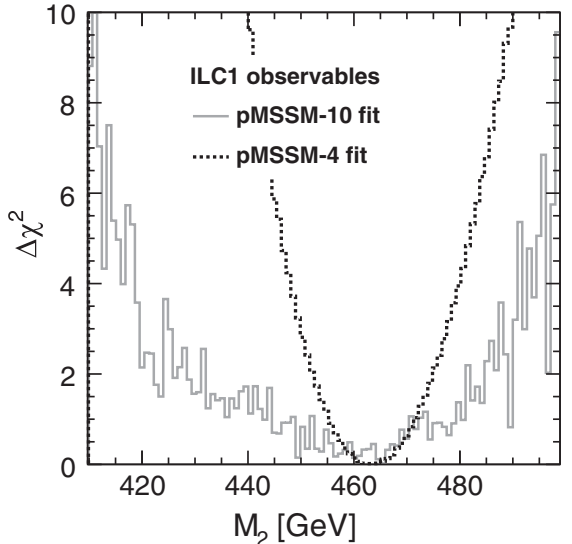

(b)

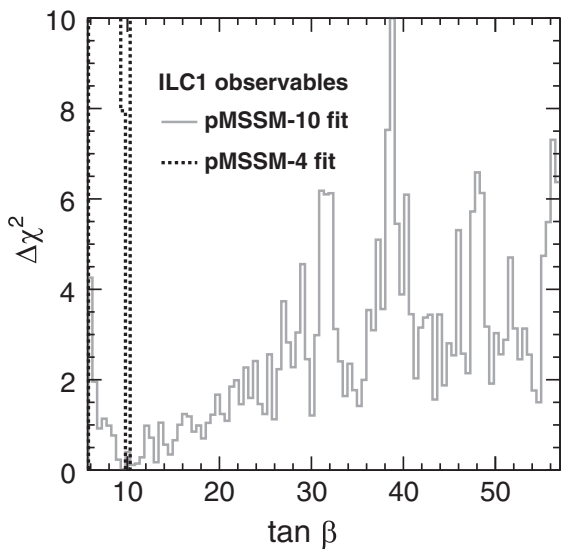

(d)

FIG. 12. ILC1: Minimum $\chi^{2}$ as a function of $M_{1}, M_{2}$, and $\mu$ and $\tan \beta$ in pMSSM-4 fit (dashed black line) and pMSSM-10 fit (solid grey line). For each bin, the minimum $\chi^{2}$ of all Markov chain points, which have the $x$ axis quantity in that bin is plotted. 
TABLE XV. Fitted parameters in ILC1 pMSSM-4, and pMSSM-10. All units in GeV except for $\tan \beta$ and $\chi^{2}$.

\begin{tabular}{|c|c|c|c|c|c|c|c|}
\hline \multirow[b]{2}{*}{ Parameter } & \multirow{2}{*}{$\begin{array}{c}\text { ILC1 } \\
\text { pMSSM } \\
\text { true }\end{array}$} & \multicolumn{3}{|c|}{ pMSSM-4 } & \multicolumn{3}{|c|}{ pMSSM-10 } \\
\hline & & $\begin{array}{l}\text { best fit } \\
\text { point }\end{array}$ & $1 \sigma \mathrm{CL}$ & $2 \sigma \mathrm{CL}$ & $\begin{array}{c}\text { best fit } \\
\text { point }\end{array}$ & $1 \sigma \mathrm{CL}$ & $2 \sigma \mathrm{CL}$ \\
\hline$M_{1}$ & 250 & 250.2 & $\begin{array}{l}+8.2 \\
-7.7\end{array}$ & $\begin{array}{l}+17.1 \\
{ }_{-15.1}\end{array}$ & 251.3 & $\begin{array}{r}+8.6 \\
-15.7\end{array}$ & $\begin{array}{l}+17.2 \\
{ }_{-23.7}\end{array}$ \\
\hline$M_{2}$ & 463 & 463.3 & $\begin{array}{l}+8.0 \\
-8.1\end{array}$ & $\begin{array}{l}+16.2 \\
-14.9\end{array}$ & 465.8 & $\begin{array}{l}+24.2 \\
-23.0\end{array}$ & $\begin{array}{l}+31.4 \\
-49.8\end{array}$ \\
\hline$\mu$ & 115.0 & 115.0 & $\begin{array}{l}+0.2 \\
-0.2\end{array}$ & $\begin{array}{l}+0.3 \\
+0.3\end{array}$ & 115.7 & +10.9 & $\begin{array}{l}+20.3 \\
+6.1\end{array}$ \\
\hline $\tan \beta$ & 10.0 & 10.0 & $\begin{array}{l}-0.2 \\
+0.1 \\
-0.1\end{array}$ & $\begin{array}{l}-0.3 \\
+0.2 \\
-0.2\end{array}$ & 9.7 & $\begin{array}{l}-4.1 \\
+8.8 \\
-3.0\end{array}$ & $\begin{array}{l}-6.1 \\
+45.3 \\
-3.5\end{array}$ \\
\hline$m_{A}$ & 1000 & & & & 1050 & $\begin{array}{l}+310 \\
-180\end{array}$ & $\begin{array}{l}+607 \\
-296\end{array}$ \\
\hline$M_{3}$ & 1270 & & & & 1412 & $\begin{array}{l}+1791 \\
-1104\end{array}$ & $\begin{array}{l}+1411 \\
{ }_{-2843}\end{array}$ \\
\hline$M_{L}$ & 7150 & & & & 7063 & $\begin{array}{l}+2029 \\
-4311\end{array}$ & $\begin{array}{r}+2645 \\
-5632\end{array}$ \\
\hline$M_{U(3)}$ & 1670 & & & & 1751 & $\begin{array}{l}+2414 \\
-628\end{array}$ & $\begin{array}{l}+4498 \\
+-740\end{array}$ \\
\hline$M_{Q(3)}$ & 4820 & & & & 4951 & $\begin{array}{l}+2324 \\
+3226\end{array}$ & $\begin{array}{l}+3858 \\
+3256\end{array}$ \\
\hline$A_{t=b=\tau}$ & -4400 & & & & -4591 & $\begin{array}{c}+1371 \\
-973\end{array}$ & $\begin{array}{l}+1647 \\
-2949\end{array}$ \\
\hline$\chi^{2}$ & & 0.0011 & & & 0.1360 & & \\
\hline
\end{tabular}

with assuming a gluino mass measurement from the LHC. As expected from Fig. 13, the effect of the gluino measurement on the other predicted masses is small.

Figure 15 illustrates the result of the corresponding pMSSM-4 fit with $M_{1}, M_{2}, \mu$, and $\tan \beta$ only. All four parameters can be determined accurately as also shown in Table XV. This results in predictions for the masses of the heavier electroweakinos with precisions between $1.6 \%$ and $3 \%$.

\section{ILC2 benchmark}

In the case of the ILC2 benchmark, the overall situation is similar to the case of ILC1. The minimum $\chi^{2}$ as a function of $M_{1}, M_{2}$ and $\mu$ and $\tan \beta$ is displayed in Fig. 16

TABLE XVI. True and fitted masses as well as their uncertainties from a pMSSM-10 fit to ILC1 observables. All values in $\mathrm{GeV}$.

\begin{tabular}{|c|c|c|c|c|}
\hline \multirow[b]{2}{*}{ Prediction } & \multicolumn{4}{|c|}{ ILC1 } \\
\hline & Model value & best fit & $1 \sigma \mathrm{CL}$ & $2 \sigma \mathrm{CL}$ \\
\hline$m_{\tilde{\chi}_{3}^{0}}$ & 265.4 & 267 & $\begin{array}{l}+8 \\
-16\end{array}$ & $\begin{array}{l}+16 \\
-26\end{array}$ \\
\hline$m_{\tilde{\chi}_{4}^{0}}$ & 521.4 & 524 & $\begin{array}{l}+20 \\
-26\end{array}$ & $\begin{array}{l}+26 \\
-55\end{array}$ \\
\hline$m_{\tilde{\chi}_{2}^{ \pm}}$ & 521.2 & 524 & $\begin{array}{l}+19 \\
-26\end{array}$ & $\begin{array}{l}+25 \\
+55\end{array}$ \\
\hline$m_{H_{0}}$ & 1001 & 1050 & $\begin{array}{r}+310 \\
+190\end{array}$ & $\begin{array}{r}+610 \\
-290\end{array}$ \\
\hline$m_{A_{0}}$ & 1000 & 1050 & $\begin{array}{l}+310 \\
-190\end{array}$ & $\begin{array}{l}+610 \\
-290\end{array}$ \\
\hline$m_{H^{ \pm}}$ & 1008 & 1056 & $\begin{array}{r}+304 \\
-176\end{array}$ & $\begin{array}{l}+604 \\
-276\end{array}$ \\
\hline$m_{\tilde{u}_{L}}$ & 7229 & 7143 & $\begin{array}{l}+2037 \\
{ }_{-4343}\end{array}$ & $\begin{array}{l}+2657 \\
-5603\end{array}$ \\
\hline$m_{\tilde{u}_{R}}$ & 7203 & 7117 & $\begin{array}{l}+2023 \\
-4337\end{array}$ & $\begin{array}{l}+2643 \\
-5577\end{array}$ \\
\hline$m_{\tilde{t}_{1}}$ & 1906 & 2003 & $\begin{array}{l}+1857 \\
-763\end{array}$ & $\begin{array}{r}+3957 \\
-803\end{array}$ \\
\hline$m_{\tilde{t}_{2}}$ & 4903 & 5033 & $\begin{array}{l}+2347 \\
-1993\end{array}$ & $\begin{array}{l}+3947 \\
-2653\end{array}$ \\
\hline$m_{\tilde{b}_{1}}$ & 4899 & 5028 & $\begin{array}{l}+2352 \\
-3188\end{array}$ & $\begin{array}{r}+3912 \\
-3488\end{array}$ \\
\hline$m_{\tilde{b}_{2}}$ & 7216 & 7130 & $\begin{array}{l}+2030 \\
+4310\end{array}$ & $\begin{array}{r}+2650 \\
-4470\end{array}$ \\
\hline$m_{\tilde{g}}$ & 1539 & 1693 & $\begin{array}{l}+1807 \\
-1273\end{array}$ & $\begin{array}{l}+2827 \\
-1693\end{array}$ \\
\hline
\end{tabular}

for the pMSSM-10 and pMSSM-4. However, this time the I20 running scenario was assumed, cf. Sec. IV C. Also here, the resulting curve for the four-parameter fit is much smoother than for the ten-parameter version due to the much smaller parameter space to be sampled. Like for ILC1, the precision on $M_{2}$ improves somewhat in the pMSSM-4 fit, while $\mu$ and $\tan \beta$ are significantly better constrained.

The resulting best fit values for the pMSSM parameters and their 1 and $2 \sigma$ intervals are compared to the input values in Table XVII, quantifying the effect which could already be seen qualitatively in Fig. 16. Again constraints

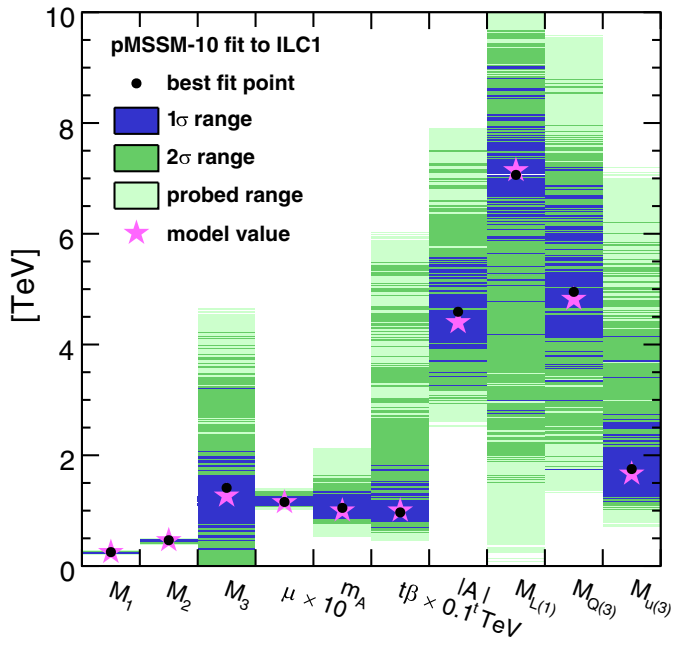

(a) without gluino observation

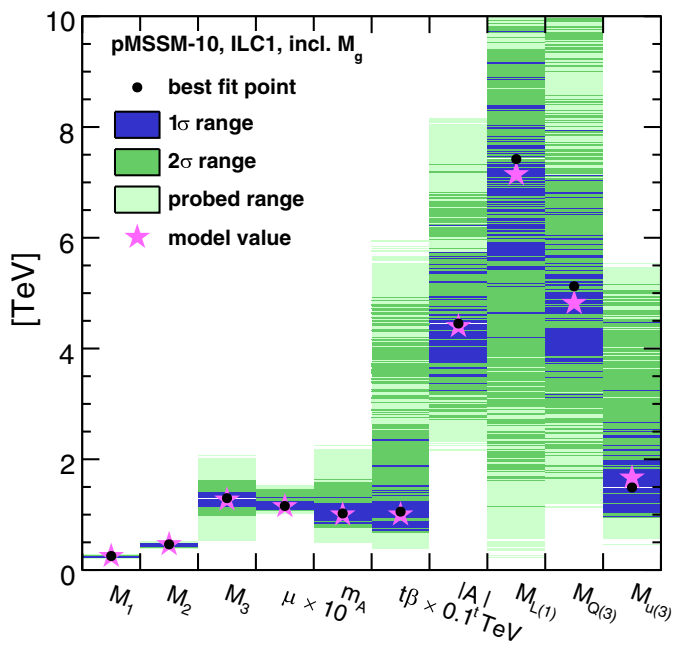

(b) with gluino observation

FIG. 13. Predicted SUSY parameter ranges from the pMSSM-10 fit to ILC1. The magenta star indicates the true model values, while the black dot shows the best fit point. 


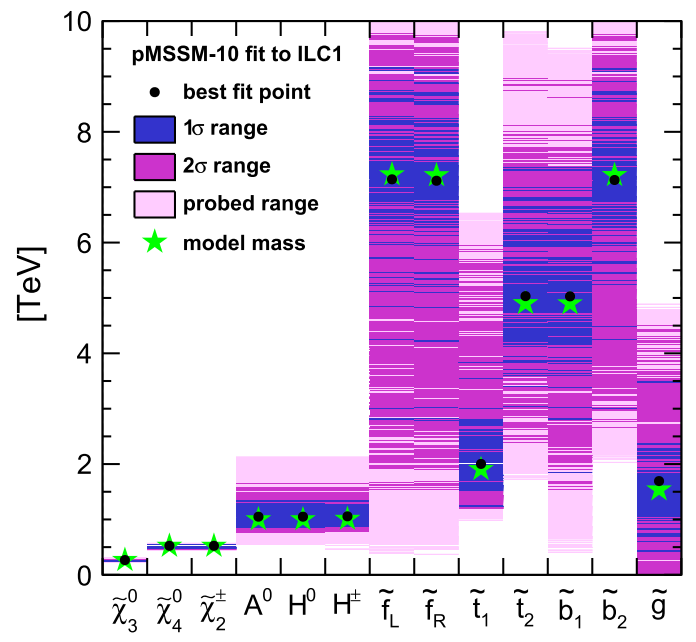

(a) without gluino observation

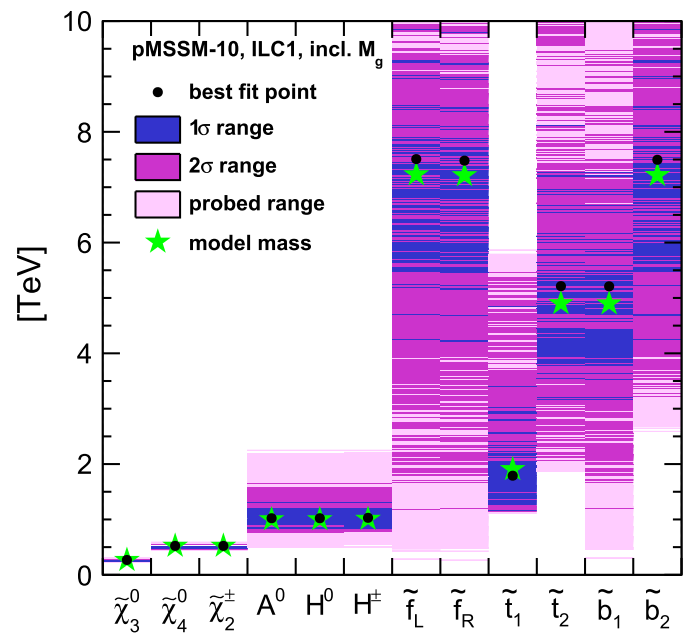

(b) with gluino observation

FIG. 14. Predicted mass ranges from the pMSSM-10 fit to ILC1. The green star indicates the true model values, while the black dot shows the best fit point.

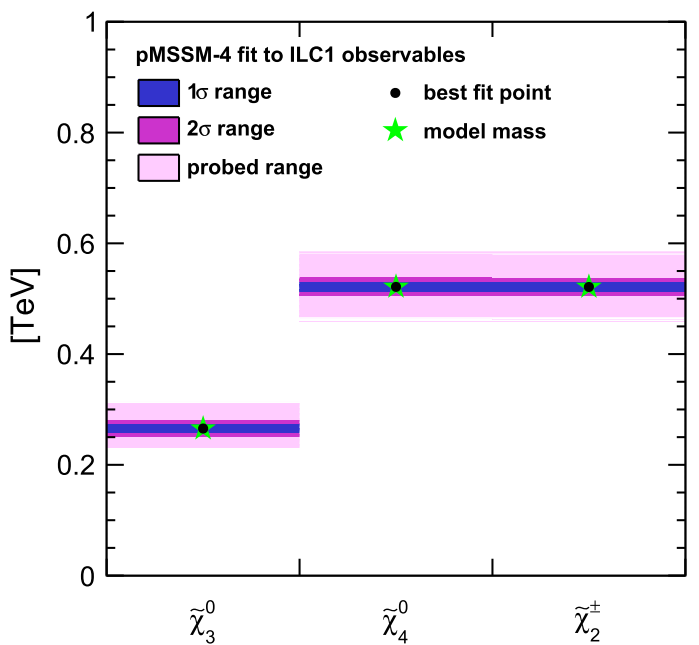

(a) Fitted masses

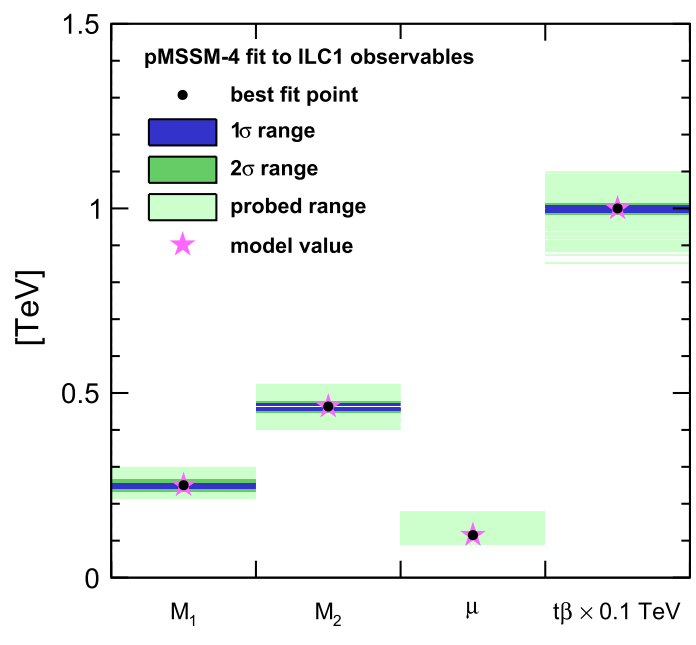

(b) Fitted parameters

FIG. 15. Predicted mass and SUSY parameter ranges from the pMSSM-4 fit to ILC1. The green/magenta star indicates the true model values, while the black dot shows the best fit point.

on the sfermion sector can be derived due to their loop contributions. In contrast to the perfect agreement of the best fit point with the input parameter values in ILC1, the best fit point for ILC2 visibly overestimates the sfermion mass parameters. However, the true values still remain within the $1 \sigma$ interval. Figure 17(b) displays the precisions obtained on the pMSSM-10 parameters. In the case of ILC2, the gluino is most likely outside the reach of LHC; therefore, $M_{3}$ is only constrained via its loop effects on the Higgsino sector.

As in the ILC1 case, the determined parameters can be used to predict the masses of the as-yet unobserved sparticles, as shown for the pMSSM-10 fit in Table XVIII and Fig. 17(a). Finally, Fig. 18(a) shows the result of the four parameter fit of $M_{1}, M_{2}, \mu, \tan \beta$. Again, the remaining parameters fixed to model values, based on the assumption that the best fit point of the ten-parameter fit is sufficiently close to the true point that the effect of fixing to the true values is negligible.

\section{3. $n G M M 1$ benchmark}

Finally, Fig. 19 shows the minimum $\chi^{2}$ as a function of $M_{1}, M_{2}$ and $\mu$ and $\tan \beta$ in the pMSSM- 10 and pMSSM- 4 fits to the nGMM1 observables. Also here, the much smaller parameter space to be sampled in case of the 


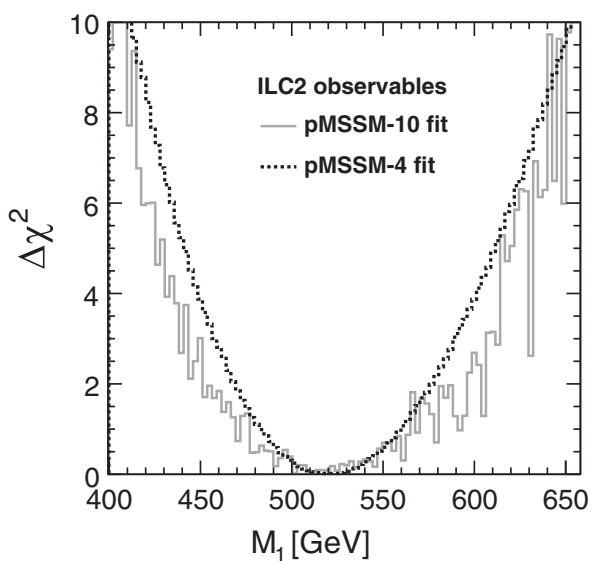

(a)

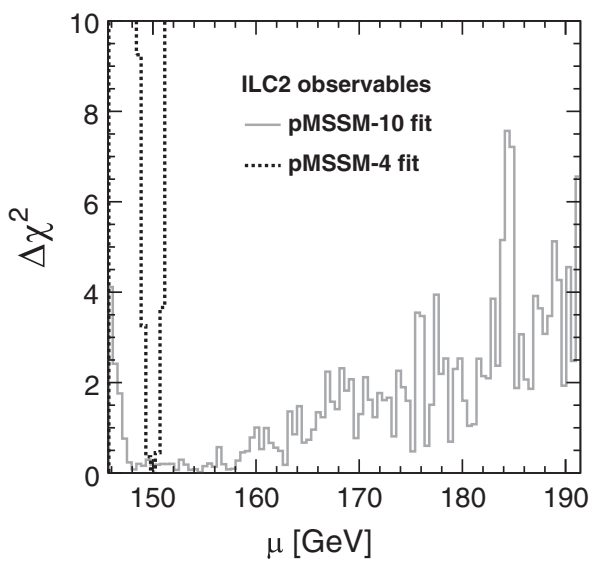

(c)

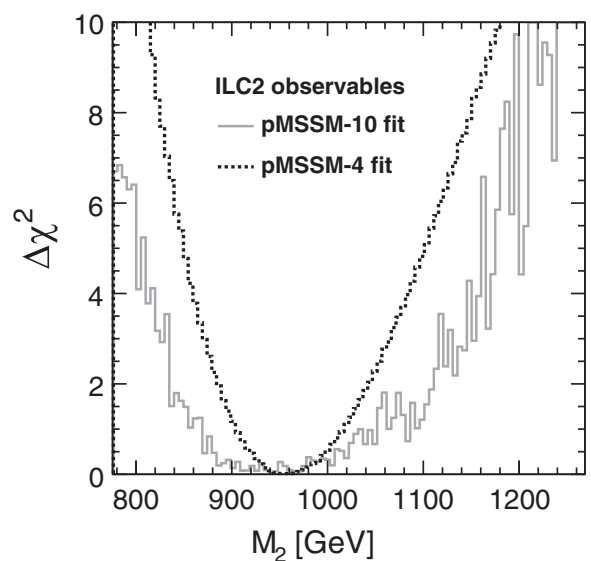

(b)

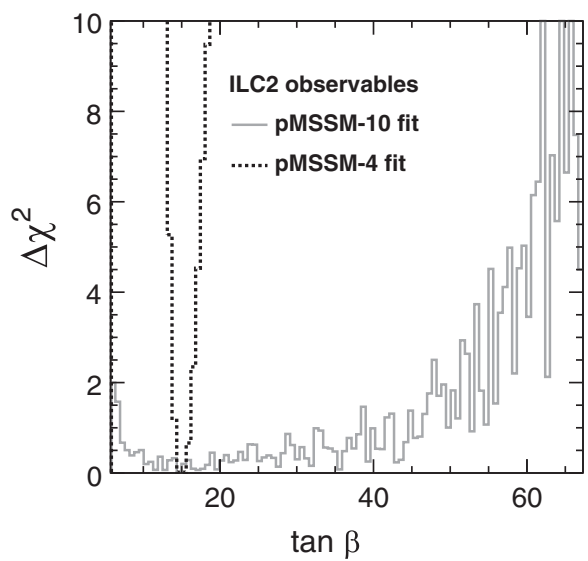

(d)

FIG. 16. ILC2: Minimum $\chi^{2}$ as a function of $M_{1}, M_{2}$ and $\mu$ and $\tan \beta$ in pMSSM-4 fit (dashed black line) and pMSSM-10 fit (solid grey line). For each bin, the minimum $\chi^{2}$ of all Markov chain points which have the $x$ axis quantity in that bin is plotted.

TABLE XVII. Fitted parameters in ILC2 pMSSM-4 and pMSSM-10. All units in GeV except for $\tan \beta$ and $\chi^{2}$.

\begin{tabular}{|c|c|c|c|c|c|c|c|}
\hline \multirow[b]{2}{*}{ Parameter } & \multirow{2}{*}{$\begin{array}{c}\text { ILC2 } \\
\text { pMSSM } \\
\text { true }\end{array}$} & \multicolumn{3}{|c|}{ pMSSM-4 } & \multicolumn{3}{|c|}{ pMSSM-10 } \\
\hline & & $\begin{array}{c}\text { best fit } \\
\text { point }\end{array}$ & $\begin{array}{l}1 \sigma \\
\mathrm{CL}\end{array}$ & $\begin{array}{l}2 \sigma \\
\mathrm{CL}\end{array}$ & $\begin{array}{c}\text { best fit } \\
\text { point }\end{array}$ & $\begin{array}{l}1 \sigma \\
\mathrm{CL}\end{array}$ & $\begin{array}{l}2 \sigma \\
\mathrm{CL}\end{array}$ \\
\hline$M_{1}$ & 520.3 & 520.7 & $\begin{array}{l}+38.6 \\
+37.6\end{array}$ & $\begin{array}{l}+79.1 \\
-71.0\end{array}$ & 502.1 & $\begin{array}{l}+91.3 \\
-32.9\end{array}$ & $\begin{array}{l}+130.1 \\
-71.7\end{array}$ \\
\hline$M_{2}$ & 957.2 & 959.42 & $\begin{array}{r}-3.0 \\
+55.4 \\
-53.1\end{array}$ & $\begin{array}{l}-11.0 \\
+124.1 \\
-100.1\end{array}$ & 941.0 & $\begin{array}{l}-32.9 \\
+145.4 \\
-711\end{array}$ & $\begin{array}{l}-11.2 \\
+229.2 \\
-130.9\end{array}$ \\
\hline$\mu$ & 150.0 & 150.0 & $\begin{array}{l}+0.4 \\
+0.4\end{array}$ & $\begin{array}{l}+0.7 \\
+0.8\end{array}$ & 154.4 & $\begin{array}{l}+24.7 \\
-7.3\end{array}$ & $\begin{array}{l}+36.6 \\
-82\end{array}$ \\
\hline $\tan \beta$ & 15.0 & 15.0 & $\begin{array}{l}+0.7 \\
-0.7\end{array}$ & $\begin{array}{l}+1.7 \\
+12\end{array}$ & 14.8 & $\begin{array}{l}+38.4 \\
-78\end{array}$ & $\begin{array}{l}+48.2 \\
-90\end{array}$ \\
\hline$m_{A}$ & 1000 & & & & 1043 & $\begin{array}{l}-1.8 \\
+135\end{array}$ & $\begin{array}{l}-9.0 \\
+240 \\
-325\end{array}$ \\
\hline$M_{3}$ & 2607 & & & & 2684 & $\begin{array}{l}+4990 \\
-2585\end{array}$ & $\begin{array}{l}+5670 \\
-2682\end{array}$ \\
\hline$M_{L}$ & 5146 & & & & 5797 & $\begin{array}{l}+2402 \\
-5359\end{array}$ & $\begin{array}{l}+3511 \\
-5544\end{array}$ \\
\hline$M_{U(3)}$ & 1395 & & & & 2073 & $\begin{array}{l}+3518 \\
-1805\end{array}$ & $\begin{array}{l}+4716 \\
-1805\end{array}$ \\
\hline$M_{Q(3)}$ & 3757 & & & & 4871 & $\begin{array}{l}+3680 \\
-3933\end{array}$ & $\begin{array}{l}+5030 \\
-4608\end{array}$ \\
\hline$A_{t}$ & -4714 & & & & -5948 & $\begin{array}{l}+2734 \\
-3387\end{array}$ & $\begin{array}{l}+3250 \\
-4050\end{array}$ \\
\hline$\chi^{2}$ & & 0.0026 & & & 0.1627 & & \\
\hline
\end{tabular}

four-dimensional fit leads to much smoother curves than in the ten-dimensional case. Again, the determinations of $\mu$ and $\tan \beta$ improve significantly. However, $M_{1}$ and $M_{2}$ exchange their roles compared to the other benchmarks, so that now $M_{1}$ is somewhat better constrained in the pMSSM-4 fit, while the precision on $M_{2}$ is nearly identical in the two fits. However, it should be noted that $M_{1}$ and $M_{2}$ are less well constrained than in the cases of the ILC1 and ILC2 benchmarks. This results from a combination of the worse experimental resolutions and the larger absolute values of $M_{1}$ and $M_{2}$ in case of nGMM1. In this most challenging case, the mass splitting between $\tilde{\chi}_{1}^{ \pm}$and $\tilde{\chi}_{1}^{0}$ is only $2.5 \mathrm{GeV}$, which corresponds to less than $2 \sigma$ of the experimental resolution. As discussed in Sec. III C, the mass differences $m_{\tilde{\chi}_{2}^{0}}-m_{\tilde{\chi}_{1}^{0}}$ and $m_{\tilde{\chi}_{1}^{ \pm}}-m_{\tilde{\chi}_{1}^{0}}$ are directly accessible experimentally as the end point of the dilepton or dijet invariant mass spectrum. Therefore, we consider in this case as alternative input, these mass differences in 


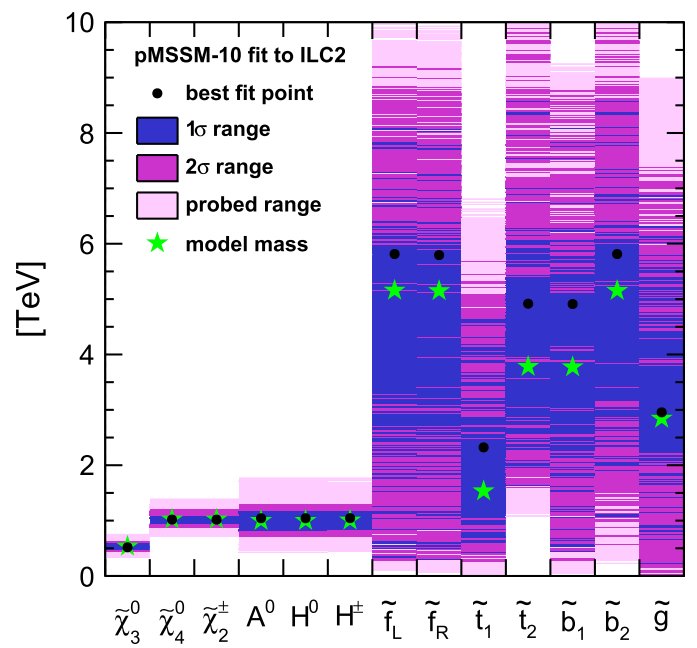

(a) Fitted masses

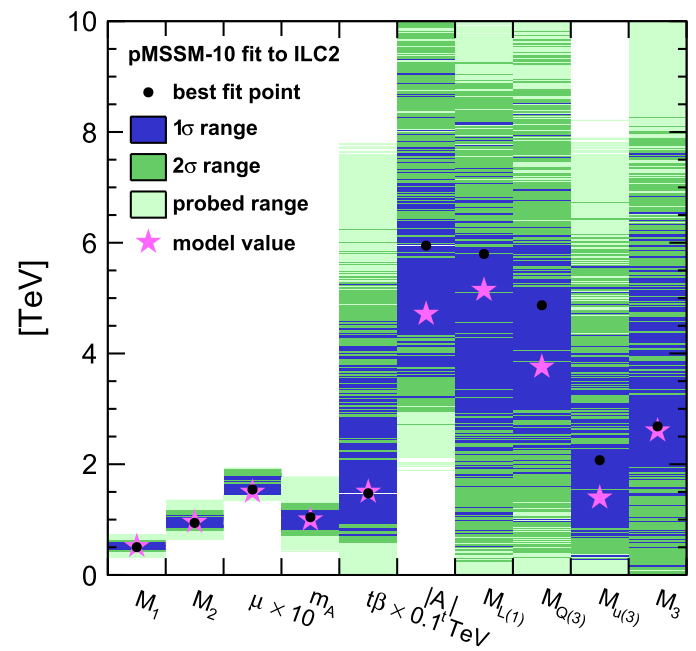

(b) Fitted parameters

FIG. 17. Predicted mass and SUSY parameter ranges from the pMSSM-10 fit to ILC2. The green/magenta star indicates the true model values, while the black dot shows the best fit point.

addition to the $\tilde{\chi}_{1}^{0}$ mass, which also presents a set of observables with minimal correlations. ${ }^{12}$ The corresponding precisions are summarized in Table XIX.

As can be seen in Fig. 20, the determination of $M_{2}$ in the ten-parameter fit improves significantly when instead of the absolute masses the mass differences are used as fit input, especially the upper bound. There is no significant effect on $M_{1}, \mu$, or $\tan \beta$.

The resulting best fit values for the pMSSM parameters and their 1 and $2 \sigma$ intervals are compared to the input values in Table XX, quantifying the effect which could already be seen qualitatively in Figs. 19 and 20. As before, constraints on the sfermion sector can be derived due to their loop contributions. In contrast to the perfect agreement of the best fit point with the input parameter values in ILC1, the best fit point for nGMM1 visibly overestimates the sfermion mass parameters. However, the true values still remain within the $1 \sigma$ interval. Using the mass differences as input, instead of the absolute Higgsino masses, notably improves the precision on $M_{2}$, as expected from the $\chi^{2}$ distribution, but it also significantly improves the agreement of the best fit point with the true model parameters. These improvements can also be seen in Figs. 21(b) and 21(d), in particular, the better agreement in $m_{A}$ and $A_{t}$.

Again, the determined parameters can be used to predict the masses of the yet unobserved sparticles, as shown for the pMSSM-10 fit in Table XXI and in Figs. 21(a) and 21(c) with standard input and when using the mass differences instead. As expected, the improved precision on $M_{2}$ when using the mass differences as input

\footnotetext{
${ }^{12}$ Note that FITTINO does not offer the option to input a covariance matrix of all input quantities and assumes all inputs as uncorrelated.
}

leads to improved predictions of the $\tilde{\chi}_{3}^{0}$ and $\tilde{\chi}_{2}^{ \pm}$ masses. In addition, the agreement between the best fit predictions for the heavy Higgs boson masses as well as for all the sfermion masses with their true value improves significantly due to the better agreement in $m_{A}$ and $A_{t}$.

Finally, Fig. 22(a) shows the result of the four parameter fit of $M_{1}, M_{2}, \mu, \tan \beta$. Again, the remaining parameters are fixed to their model values, based on the assumption that the best fit point of the ten-parameter fit is sufficiently close to the true point that the effect of fixing to the true values is negligible. The masses of the heavier electroweakinos are

TABLE XVIII. True and fitted masses as well as their uncertainties from a pMSSM-10 fit to ILC2 observables. All values in $\mathrm{GeV}$.

\begin{tabular}{|c|c|c|c|c|}
\hline \multirow[b]{2}{*}{ Prediction } & \multicolumn{4}{|c|}{ ILC2 } \\
\hline & Model masses & best fit & $1 \sigma$ & $2 \sigma$ \\
\hline$m_{\tilde{\chi}_{3}^{0}}$ & 534.6 & 518 & $\begin{array}{l}+72 \\
-34\end{array}$ & $\begin{array}{l}+110 \\
-74\end{array}$ \\
\hline$m_{\tilde{\chi}_{4}^{0}}$ & 1026 & 1018 & $\begin{array}{l}+82 \\
-76\end{array}$ & $\begin{array}{l}+190 \\
-134\end{array}$ \\
\hline$m_{\tilde{\chi}_{2}^{ \pm}}$ & 1026 & 1018 & $\begin{array}{l}+82 \\
-76\end{array}$ & $\begin{array}{l}+190 \\
{ }_{-134}\end{array}$ \\
\hline$m_{H_{0}}$ & 1000 & 1043 & $\begin{array}{l}+137 \\
{ }_{-223}\end{array}$ & $\begin{array}{l}+257 \\
-323\end{array}$ \\
\hline$m_{A_{0}}$ & 1000 & 1043 & $\begin{array}{l}+137 \\
-223\end{array}$ & $\begin{array}{l}+257 \\
-323\end{array}$ \\
\hline$m_{H^{ \pm}}$ & 1003 & 1045 & $\begin{array}{l}+135 \\
-205\end{array}$ & $\begin{array}{l}+255 \\
-325\end{array}$ \\
\hline$m_{\tilde{u}_{L}}$ & 5158 & 5814 & $\begin{array}{l}+2286 \\
-5474\end{array}$ & $\begin{array}{l}+3406 \\
-5534\end{array}$ \\
\hline$m_{\tilde{u}_{R}}$ & 5143 & 5795 & $\begin{array}{l}+2285 \\
-5495\end{array}$ & $\begin{array}{l}+3385 \\
-5495\end{array}$ \\
\hline$m_{\tilde{t}_{1}}$ & 1535 & 2322 & $\begin{array}{l}+2318 \\
-1902\end{array}$ & $\begin{array}{l}+3378 \\
-2062\end{array}$ \\
\hline$m_{\tilde{t}_{2}}$ & 3782 & 4917 & $\begin{array}{l}+3663 \\
-3277\end{array}$ & $\begin{array}{l}+4983 \\
-3317\end{array}$ \\
\hline$m_{\tilde{b}_{1}}$ & 3774 & 4911 & $\begin{array}{r}+311 \\
+3471\end{array}$ & $\begin{array}{r}-3869 \\
+3631\end{array}$ \\
\hline$m_{\tilde{b}_{2}}$ & 5154 & 5814 & $\begin{array}{l}-4411 \\
+2766\end{array}$ & $\begin{array}{l}-4631 \\
+4086\end{array}$ \\
\hline$m_{\tilde{g}}$ & 2846 & 2955 & $\begin{array}{l}-4734 \\
+3925 \\
-2735\end{array}$ & $\begin{array}{l}-5254 \\
+4445 \\
-2025\end{array}$ \\
\hline
\end{tabular}




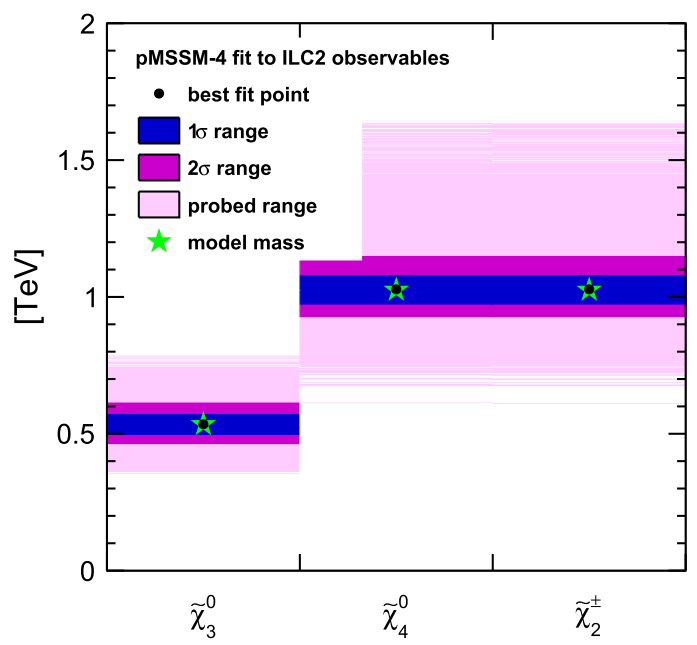

(a) Fitted masses

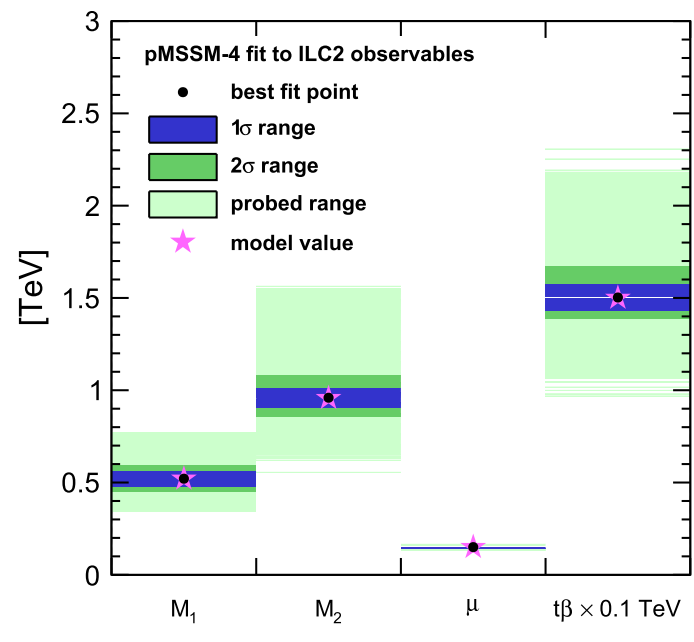

(b) Fitted parameters

FIG. 18. Predicted mass and SUSY parameter ranges from the pMSSM-4 fit to ILC2. The green/magenta star indicates the true model values, while the black dot shows the best fit point.

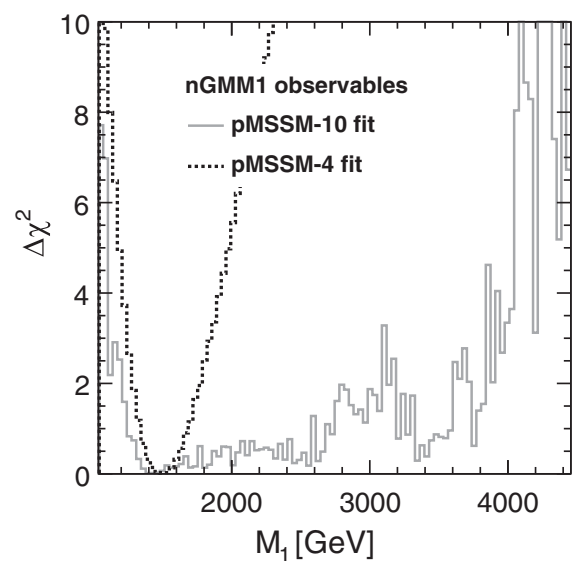

(a)

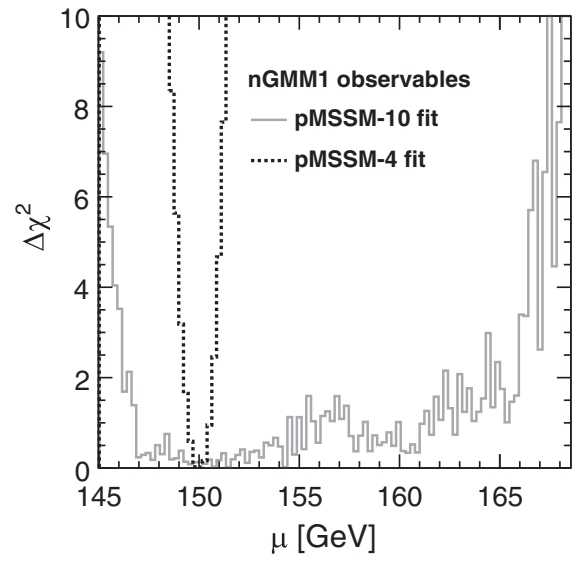

(c)

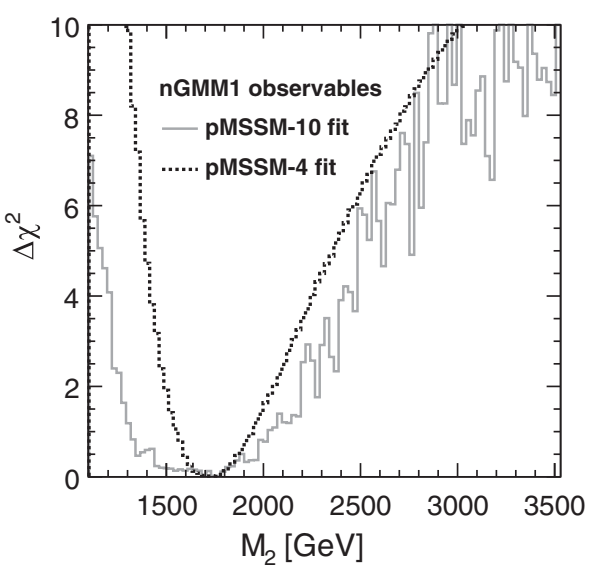

(b)

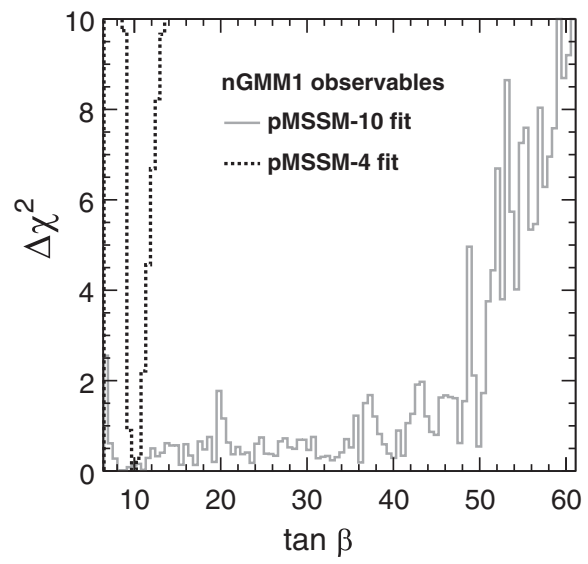

(d)

FIG. 19. nGMM1: Minimum $\chi^{2}$ as a function of $M_{1}, M_{2}$ and $\mu$ and $\tan \beta$ in pMSSM-4 fit (dashed black line) and pMSSM-10 fit (solid grey line). For each bin, the minimum $\chi^{2}$ of all Markov chain points which have the $x$ axis quantity in that bin is plotted. 


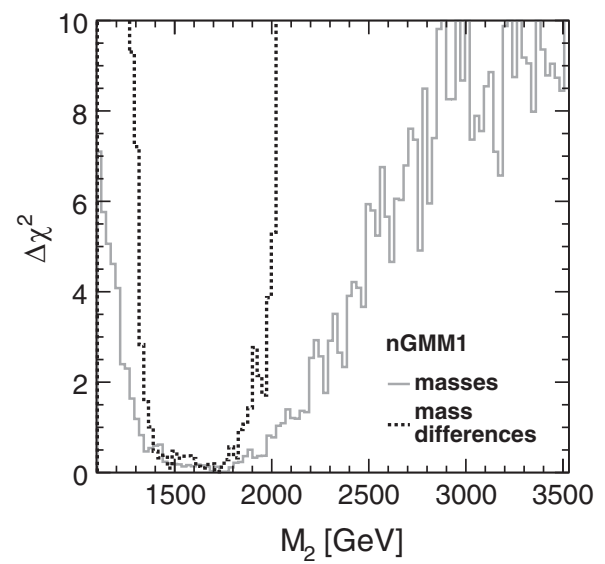

FIG. 20. nGMM1 $\chi^{2}$ parabola for $M_{2}$ in the ten-parameter fit with mass differences (black, dashed) and the same fit with masses as observables (grey, solid).

predicted within an $1 \sigma$ uncertainty of about $150 \mathrm{GeV}$. This fit has only been run with the standard input; further improvements could be expected when using the mass differences as input also in this fit.

TABLE XIX. Experimental precision on the Higgsino mass differences in nGMM1 combined from $500 \mathrm{GeV} 500^{-1} \mathrm{fb}$ for both $\mathcal{P}( \pm 0.8, \mp 0.3)$, as well as scaled to $1600 \mathrm{fb}^{-1}$ for both polarizations at $\sqrt{s}=500 \mathrm{GeV}$, ignoring the data sets with other centerof-mass energies in the I20 operating scenario. Again, it is assumed that the precisions obtained from the simulations based on the ISAJET spectrum can be transferred to the SPHENO3.3.9BETA spectrum.

\begin{tabular}{lccc}
\hline \hline Observable & $\begin{array}{c}\text { nGMM1 model } \\
\text { value }[\mathrm{GeV}]\end{array}$ & Precision & I20 precision \\
\hline$m_{\tilde{\chi}_{1}^{0}}$ & 154.9 & $1.7 \%$ & $1.0 \%$ \\
$m_{\tilde{\chi}_{2}^{0}}-m_{\tilde{\chi}_{1}^{0}}$ & 5.3 & $2.1 \%$ & $1.4 \%$ \\
$m_{\tilde{\chi}_{1}^{ \pm}}-m_{\tilde{\chi}_{1}^{0}}$ & 2.4 & $2.5 \%$ & $1.2 \%$ \\
\hline \hline
\end{tabular}

\section{Dark matter in Higgsino fits}

An additional benefit from our fits to MSSM parameters is that it is possible to extract various WIMP dark matter related observables [66]. These include 1 . the thermally produced WIMP relic density $\Omega_{\tilde{\chi}}^{\mathrm{TP}} h^{2}, 2$. the spin-dependent (SD) and spin-independent (SI) WIMP-nucleon scattering cross section [e.g., $\sigma^{\mathrm{SI}}(\chi p)$ ] which is constrained by WIMP direct detection search experiments, and 3. the thermally averaged WIMP-WIMP annihilation cross section times relative velocity (evaluated as $v \rightarrow 0$ ) $\langle\sigma v\rangle$ which is constrained by indirect WIMP search results, which look for cosmic WIMP-WIMP annihilation to high energy photons and antimatter. The theory predictions for these observables from ISARED [67] and ISARES [68] are listed in Table I. The Higgsino-like WIMPs are thermally underproduced as dark matter and if their abundance is augmented via nonthermal WIMP production, then the Higgsino-like WIMPs are excluded by direct and indirect WIMP search experiments [69]. However, by requiring naturalness in the QCD sector (i.e., the axionic solution to the strong $C P$ problem) as well as in the electroweak sector, then we are led to require the presence of axionic dark matter as well. Thus, from naturalness, we expect two dark matter particles: axions as well as Higgsino-like WIMPs. In fact, detailed calculations using eight coupled Boltzmann equations (which track axion, WIMP, axino, saxion, gravitino, and radiation abundances) suggest that the axions usually dominate the dark matter abundance [70]. Then the diminished presence of Higgsinos in the relic DM density leads to consistency with WIMP search results since there are fewer Higgsinos present in the relic abundance (typically $10 \%-20 \%)$ than is usually assumed (100\%).

To obtain these fitted values, we use FITTINo [29] together with MICROMEGAS [71] and ASTROFIT [72]. The fitted and scaled relic density is plotted, and the $2 \sigma$ confidence interval has been extracted. The center of

TABLE XX. Fitted parameters in nGMM1: pMSSM-4, pMSSM-10, and pMSSM-10 with mass differences as input. All units in GeV except for $\tan \beta$ and $\chi^{2}$.

\begin{tabular}{|c|c|c|c|c|c|c|c|c|c|c|}
\hline \multirow[b]{2}{*}{ Parameter } & \multirow[b]{2}{*}{ True } & \multicolumn{3}{|c|}{ pMSSM-4 } & \multicolumn{3}{|c|}{ pMSSM-10 } & \multicolumn{3}{|c|}{ pMSSM-10 with mass differences } \\
\hline & & Best fit point & $1 \sigma \mathrm{CL}$ & $2 \sigma \mathrm{CL}$ & Best fit point & $1 \sigma \mathrm{CL}$ & $2 \sigma \mathrm{CL}$ & Best fit point & $1 \sigma \mathrm{CL}$ & $2 \sigma \mathrm{CL}$ \\
\hline$M_{1}$ & 1493 & 1501 & +173 & +411 & 1386 & +2386 & +2830 & 1573 & +2091 & +5650 \\
\hline$M_{2}$ & 1720 & 1711 & $\begin{array}{l}+220 \\
-158\end{array}$ & $\begin{array}{l}+530 \\
+279\end{array}$ & 1768 & $\begin{array}{l}-145 \\
+254\end{array}$ & $\begin{array}{l}-282 \\
+717 \\
-549\end{array}$ & 1710 & $\begin{array}{l}-282 \\
+137\end{array}$ & $\begin{array}{l}-344 \\
+277 \\
-394\end{array}$ \\
\hline$\mu$ & 150.0 & 150.0 & $\begin{array}{l}-108 \\
+0.4 \\
-0.4\end{array}$ & $\begin{array}{l}-219 \\
+0.9\end{array}$ & 154.2 & $\begin{array}{l}-431 \\
+7.4 \\
-87\end{array}$ & $\begin{array}{l}-349 \\
+12.9 \\
-8.3\end{array}$ & 149.9 & $\begin{array}{l}-313 \\
+11.5 \\
-3.4\end{array}$ & $\begin{array}{l}-394 \\
+15.3 \\
-4.2\end{array}$ \\
\hline $\tan \beta$ & 10.0 & 10.0 & $\begin{array}{l}+0.5 \\
+0.5\end{array}$ & $\begin{array}{l}+1.2 \\
+06\end{array}$ & 8.3 & $\begin{array}{l}-8.1 \\
+41.9\end{array}$ & $\begin{array}{l}-44.6 \\
+4.9\end{array}$ & 11.2 & $\begin{array}{l}-3.4 \\
+32.5 \\
-3.4\end{array}$ & $\begin{array}{l}-4.2 \\
+63.9\end{array}$ \\
\hline$m_{A}$ & 2000 & & & & 2655 & $\begin{array}{l}-1.3 \\
+6493 \\
-1449\end{array}$ & $\begin{array}{l}-11492 \\
+1596\end{array}$ & 1868 & $\begin{array}{l}-3.4 \\
+4018 \\
-567\end{array}$ & $\begin{array}{l}-4623 \\
+6467 \\
-867\end{array}$ \\
\hline$M_{3}$ & 2646 & & & & 3173 & $\begin{array}{l}-1449 \\
+4229 \\
-3168\end{array}$ & $\begin{array}{l}-1380 \\
+5347 \\
-3168\end{array}$ & 2677 & $\begin{array}{r}-381 \\
+3892 \\
-2541\end{array}$ & $\begin{array}{l}-601 \\
+4550 \\
-2614\end{array}$ \\
\hline$M_{L}$ & 5115 & & & & 4781 & $\begin{array}{l}-3108 \\
+3589 \\
-4077\end{array}$ & $\begin{array}{l}-3108 \\
+4630 \\
-4456\end{array}$ & 5412 & $\begin{array}{l}-2541 \\
+1629 \\
-4581\end{array}$ & $\begin{array}{l}-2614 \\
+2319 \\
-5118\end{array}$ \\
\hline$M_{U(3)}$ & 1381 & & & & 1774 & $\begin{array}{l}-4011 \\
+2384 \\
-1086\end{array}$ & $\begin{array}{l}-4430 \\
+4826 \\
-1214\end{array}$ & 996 & $\begin{array}{l}-4301 \\
+3540 \\
-500\end{array}$ & $\begin{array}{l}-5118 \\
+4686 \\
-741\end{array}$ \\
\hline$M_{Q(3)}$ & 3701 & & & & 4011 & $\begin{array}{l}-1086 \\
+3254 \\
-3535\end{array}$ & $\begin{array}{l}-1214 \\
+3982 \\
-3697\end{array}$ & 3874 & $\begin{array}{l}-500 \\
+1983 \\
-3245\end{array}$ & $\begin{array}{l}-141 \\
+2356 \\
-3370\end{array}$ \\
\hline$A_{t}$ & -4857 & & & & -6766 & $\begin{array}{l}+3698 \\
-509\end{array}$ & $\begin{array}{l}+4012 \\
-1702\end{array}$ & -4582 & $\begin{array}{l}+1558 \\
-4006\end{array}$ & $\begin{array}{l}+1750 \\
-4390\end{array}$ \\
\hline$\chi^{2}$ & & 0.0138 & & & 0.0927 & & & 0.0668 & & \\
\hline
\end{tabular}




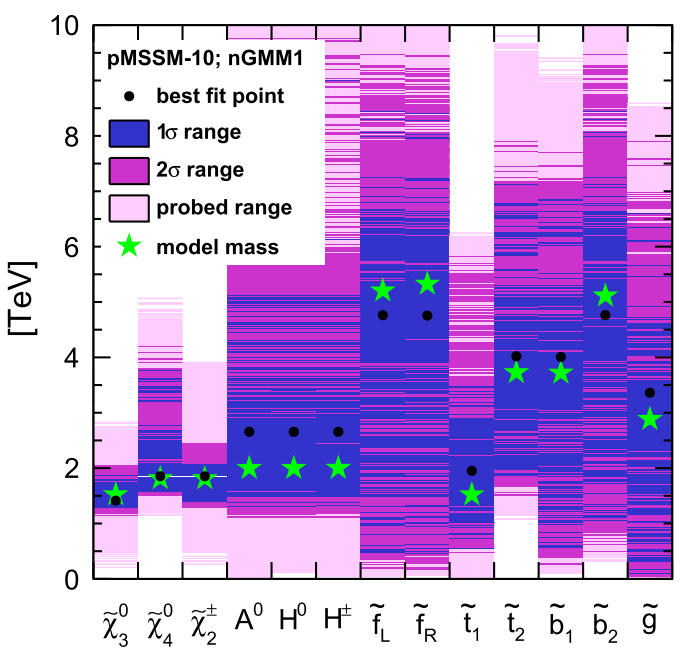

(a) Fitted masses, standard input

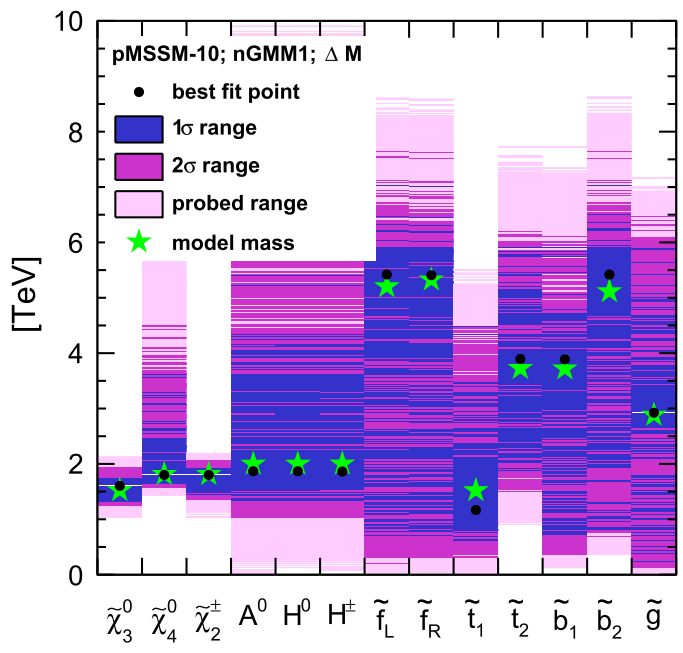

(c) Fitted masses, mass difference input

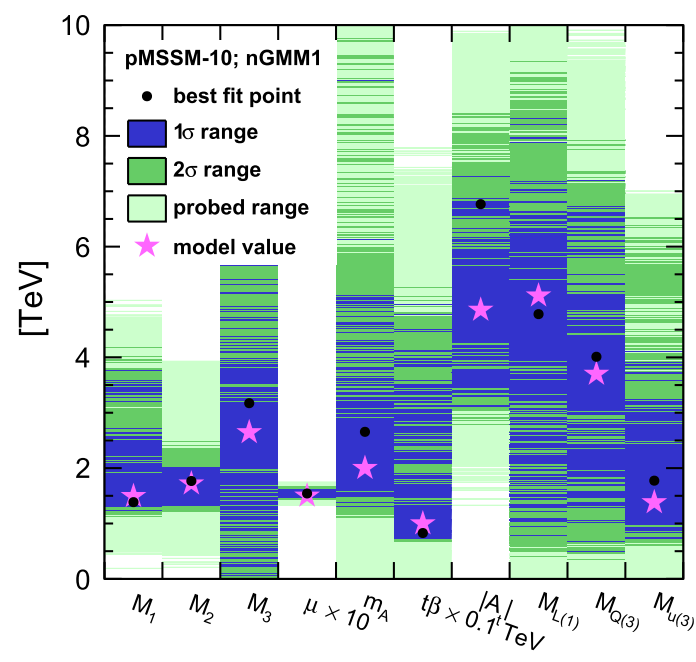

(b) Fitted parameters, standard input

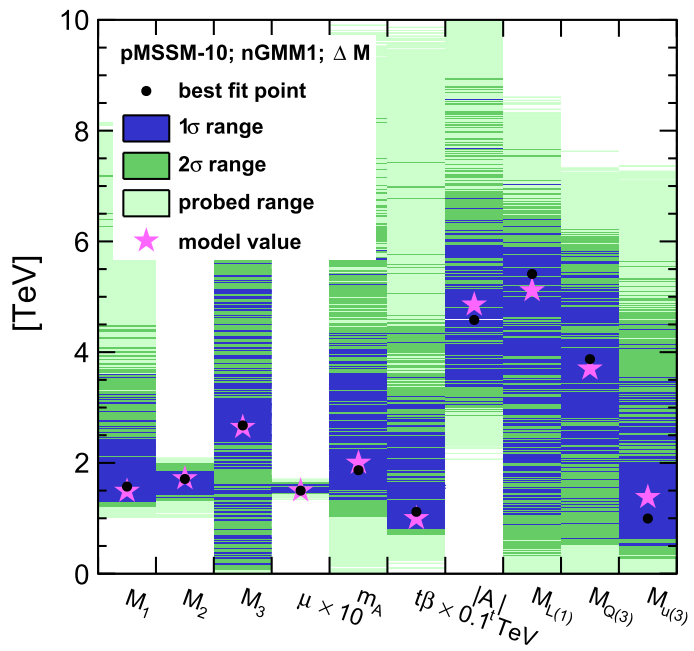

(d) Fitted parameters, mass difference input

FIG. 21. Predicted mass and SUSY parameter ranges from the pMSSM-10 fit to nGMM1 observables-including either $\tilde{\chi}_{2}^{0}$ and $\tilde{\chi}_{1}^{ \pm}$ masses or their mass differences with the LSP. The green/magenta star indicates the true model values, while the black dot shows the best fit point.

the $2 \sigma$ confidence level is calculated and used as the mean. The width of the $2 \sigma$ range is divided by two to obtain the $1 \sigma$ width assuming the $\Delta \chi^{2}$ distribution is parabolic. The distributions are more flat than parabolic so this procedure gives a conservative estimate of the $1 \sigma$ width. The relic density distribution from each fit is plotted, assuming a Gaussian distribution, in Fig. 23. In the case of the pMSSM-10 fit without any further inputs, the relic density is not sufficiently constrained. However, this has been traced to be due to fit solutions with extremely low gluino masses of less than $200 \mathrm{GeV}$. Excluding these points, the blue dashed curves are obtained, which show a very good determination of the relic density agreeing quite well with the theoretical value.
The precision improves even further when the pMSSM4 fit is run after the pMSSM10 fit. Such a measurement of the relic density would clearly confirm a possible underabundance of Higgsino-like WIMPs.

We also fit the expected values of $\sigma^{\mathrm{SI}}(\chi p)$ and $\langle\sigma v\rangle$ which are listed in Table XXII (these theory values are somewhat higher than those obtained in Table I using ISARES [68] due to ISAJET/SPHENO spectrum differences and different coding algorithms for direct/indirect detection rates). The $\sigma^{\mathrm{SI}}$ values can be fit to an accuracy typically better than $1 \%$ while the $\langle\sigma v\rangle$ values are typically fit to $\sim 10 \%$ or worse. By comparing the direct detection rates from WIMP detection experiments to the ILC fitted values for a measured Higgsino mass $m_{\chi}$, a direct measurement of WIMP relic density can be 
TABLE XXI. nGMM1: True and fitted masses and their uncertainties from pMSSM-10 fits with the standard set of observables as well as with the Higgsino mass differences replacing the $\tilde{\chi}_{2}^{0}$ and $\tilde{\chi}_{1}^{ \pm}$masses as observables. All values in $\mathrm{GeV}$.

\begin{tabular}{|c|c|c|c|c|c|c|c|}
\hline \multirow[b]{2}{*}{ Prediction } & \multicolumn{3}{|c|}{ nGMM1M } & \multicolumn{3}{|c|}{$\mathrm{nGMM} 1 \Delta M$} & \\
\hline & $\begin{array}{c}\text { Model mass } \\
\text { best fit }\end{array}$ & $1 \sigma$ & $2 \sigma$ & Best fit & $1 \sigma$ & $2 \sigma$ & \\
\hline$m_{\tilde{\chi}_{3}^{0}}$ & 1522 & 1412 & $\begin{array}{l}+454 \\
-134\end{array}$ & $\begin{array}{l}+640 \\
{ }_{-260}\end{array}$ & 1603 & $\begin{array}{l}+149 \\
-283\end{array}$ & $\begin{array}{l}+347 \\
-349\end{array}$ \\
\hline$m_{\tilde{\chi}_{4}^{0}}$ & 1809 & 1854 & $\begin{array}{l}-134 \\
+1920 \\
-264\end{array}$ & $\begin{array}{r}-2364 \\
+2364 \\
-336\end{array}$ & 1802 & $\begin{array}{r}-1834 \\
+1834\end{array}$ & $\begin{array}{r}-2710 \\
+218\end{array}$ \\
\hline$m_{\tilde{\chi}_{2}^{ \pm}}$ & 1808 & 1853 & $\begin{array}{l}-204 \\
+229 \\
-443\end{array}$ & $\begin{array}{l}-500 \\
+601 \\
-557\end{array}$ & 1801 & $\begin{array}{l}-140 \\
+137\end{array}$ & $\begin{array}{l}-210 \\
+275\end{array}$ \\
\hline$m_{H_{0}}$ & 2000 & 2655 & $\begin{array}{r}-443 \\
+6365 \\
-1355\end{array}$ & $\begin{array}{r}-531 \\
+7125 \\
\end{array}$ & 1868 & $\begin{array}{r}-3499 \\
+3992 \\
-578\end{array}$ & $\begin{array}{r}-453 \\
+6372 \\
\end{array}$ \\
\hline$m_{A_{0}}$ & 2000 & 2655 & $\begin{array}{l}-1355 \\
+6365 \\
-1355\end{array}$ & $\begin{array}{r}-1535 \\
+7125 \\
\end{array}$ & 1868 & $\begin{array}{l}-528 \\
+3992 \\
-528\end{array}$ & $\begin{array}{r}-828 \\
+6372 \\
+020\end{array}$ \\
\hline$m_{H^{ \pm}}$ & 2002 & 2656 & $\begin{array}{l}-1355 \\
+6364 \\
-1336\end{array}$ & $\begin{array}{l}-1535 \\
+7124 \\
-1556\end{array}$ & 1863 & $\begin{array}{l}-320 \\
+3997 \\
-523\end{array}$ & $\begin{array}{l}-828 \\
+6377\end{array}$ \\
\hline$m_{\tilde{u}_{L}}$ & 5121 & 4762 & $\begin{array}{l}13608 \\
+3698\end{array}$ & $\begin{array}{l}-1300 \\
+4718\end{array}$ & 5421 & $\begin{array}{r}-323 \\
+1619\end{array}$ & $\begin{array}{r}-823 \\
+2239\end{array}$ \\
\hline$m_{\tilde{\tilde{n}}}$ & 5110 & 4754 & $\begin{array}{l}-42 \\
+36\end{array}$ & $\begin{array}{l}-4582 \\
-4706\end{array}$ & 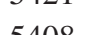 & $\begin{array}{l}-4721 \\
+161\end{array}$ & $\begin{array}{l}-5221 \\
+222\end{array}$ \\
\hline$m_{\tilde{u}}$ & & & $\begin{array}{l}-4294 \\
-4\end{array}$ & -4594 & 5408 & $\begin{array}{l}+10112 \\
-4708\end{array}$ & -5108 \\
\hline$m_{\tilde{t}_{1}}$ & 1519 & 1951 & $\begin{array}{l}+1549 \\
-1411\end{array}$ & $\begin{array}{l}+3889 \\
-1471\end{array}$ & 1168 & $\begin{array}{l}+3332 \\
-548 \\
-54\end{array}$ & $\begin{array}{l}+3332 \\
-868\end{array}$ \\
\hline$m_{\tilde{t}_{2}}$ & 3782 & 4029 & $\begin{array}{r}+3120 \\
+3160\end{array}$ & $\begin{array}{l}-3900 \\
+3900\end{array}$ & 3894 & +2026 & $\begin{array}{r}-000 \\
+2326\end{array}$ \\
\hline$m_{\tilde{b}_{1}}$ & 3774 & 4008 & $\begin{array}{r}+2852 \\
+282\end{array}$ & $\begin{array}{l}+3712 \\
+3712\end{array}$ & 3888 & $\begin{array}{r}+2032 \\
\end{array}$ & $\begin{array}{r}+2232 \\
\end{array}$ \\
\hline$m_{\tilde{b}_{2}}$ & 5154 & 4763 & $\begin{array}{l}-3448 \\
+3697\end{array}$ & $\begin{array}{r}-3748 \\
+4717\end{array}$ & 5410 & $\begin{array}{r}-3168 \\
+162\end{array}$ & $\begin{array}{r}-3528 \\
+224\end{array}$ \\
\hline & & & -3703 & -4263 & 3419 & -4599 & -4739 \\
\hline$m_{\tilde{g}}$ & 2846 & 3361 & $\begin{array}{l}+3259 \\
-3261\end{array}$ & $\begin{array}{l}+4559 \\
-3361\end{array}$ & 2924 & $\begin{array}{l}+2976 \\
-2684\end{array}$ & $\begin{array}{l}+3556 \\
-2804\end{array}$ \\
\hline
\end{tabular}

made since the WIMP direct detection rates are actually sensitive to $\xi \sigma^{\mathrm{SI}}$, where $\xi$ is the ratio of actual WIMP abundance divided by the total measured abundance $\Omega_{a+\chi} h^{2}$. Such interplay between ILC results and direct detection results offer direct confirmation that WIMPs would comprise only a portion of dark matter. In addition, indirect WIMP detection rates are proportional to $\xi^{2}$ since they search for WIMP-WIMP annihilation. The interplay of ILC results with indirect WIMP detection rates could offer further confirmation for multicomponent dark matter.

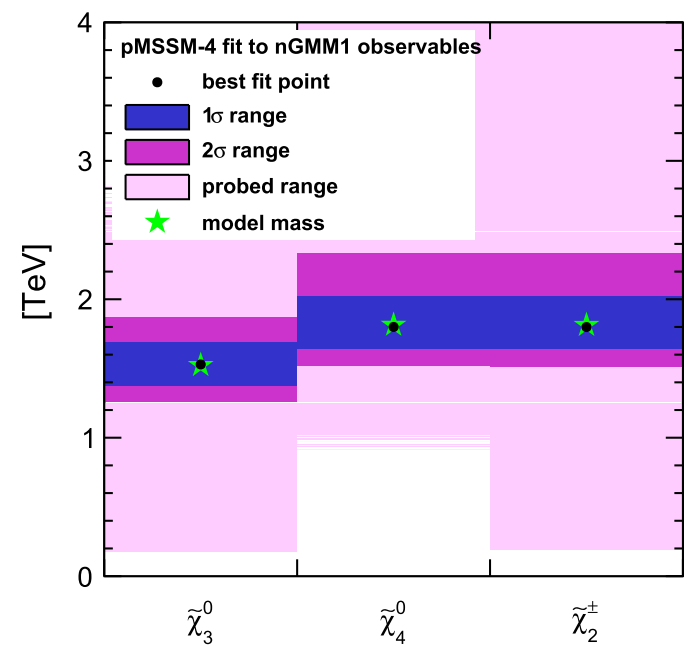

(a) Fitted masses

\section{TESTING GAUGINO MASS UNIFICATION}

The pMSSM parameters which were extracted in Sec. V B were fitted at the energy scale $Q=1 \mathrm{TeV}$. The scale dependence of the parameters is governed by their renormalization group equations or RGEs. Using the MSSM RGEs, the fitted parameters can be evolved to higher energy scales in order to check hypotheses regarding unification. Specifically, we will test the unification of the various gaugino masses which are assumed to unify at $Q=m_{\mathrm{GUT}} \simeq 2 \times 10^{16} \mathrm{GeV}$ (the scale at which gauge couplings unify) in models like NUHM2 and NUHM3 but which would unify at a lower scale in models such as nGMM1. Since in this work we do not subscribe to any particular GUT or string theory, GUT scale threshold corrections to gauge and Yukawa couplings and soft SUSY breaking terms are not imposed.

This section continues the program initiated by Blair et al. of extracting tests of high scale unification from weak scale measurements of SUSY particle properties at ILCV $[74,75]$. Since the estimates of the achievable precision for the experimental observables used in Sec. V are somewhat more pessimistic than the results obtained in Sec. IV, we also discuss the expected impact of the experimental improvements taking the nGMM1 benchmark as an example.

\section{A. Method}

The running pMSSM-10 weak scale parameters and error bars are extracted using FITTINO and SPHENO3.3.9BETA at $Q=1 \mathrm{TeV}$. Then a random scan of $10^{4}$ samples of the ten parameters is performed, approximating the parameter PDFs as either Gaussian or flat within $\pm 1 \sigma$, depending on the shape of their $\chi^{2}$ distribution; see, e.g., Fig. 19. For each of the sampled points, SPHENO was used to calculate the

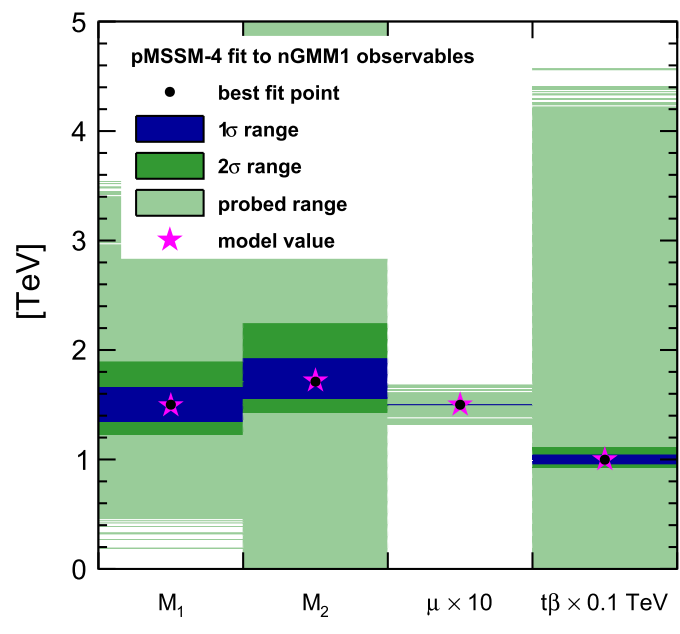

(b) Fitted parameters

FIG. 22. Predicted mass and SUSY parameter ranges from the pMSSM-4 fit to nGMM1. The green/magenta star indicates the true model values, while the black dot shows the best fit point. 


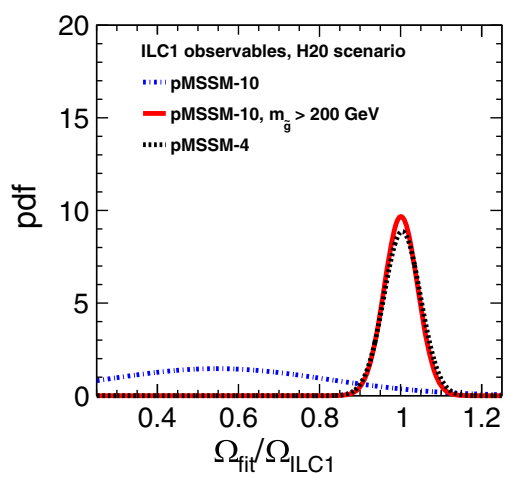

(a) ILC1

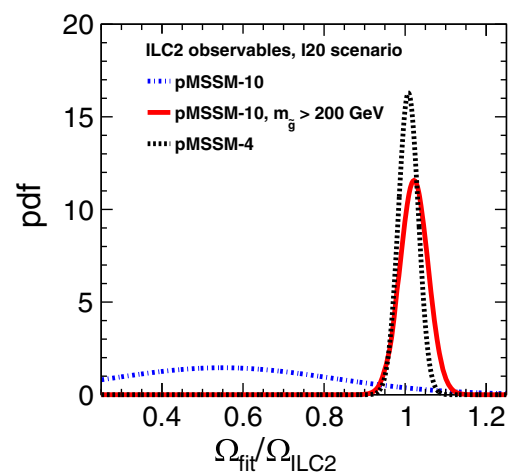

(b) ILC2

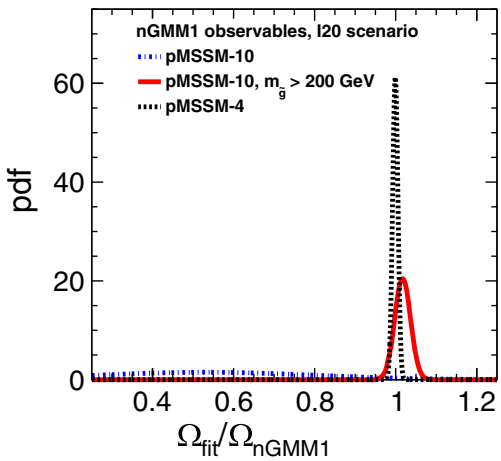

(c) nGMM1

FIG. 23. Fitted relic densities in ILC1, ILC2, and nGMM1 fits.

running parameters at each of 21 energy scales between 91 and $10^{19} \mathrm{GeV}$. The mean and standard distribution of these parameters' distributions at each energy scale were used to define confidence bands, as shown in, e.g., Fig. 22(b). The unification scale $Q_{\text {unif }}$ is determined by fitting linear functions the running parameters in a range close to the visible intersection and extracting the intersection point. With each value for $Q_{\text {unif }}$, a corresponding estimate of $M_{1 / 2}$ is determined. Gaussian functions can be fitted to the distributions of the resulting values for $Q_{\text {unif }}$ and $M_{1 / 2}$ in order to obtain central values and uncertainties.

For the gluino mass, several scenarios are considered: the determination from loop contributions to the Higgsino observables only, a direct observation at the LHC resulting in a precision of $10 \%$ on the physical gluino mass, or simply by assuming gaugino mass unification. In the latter case, the extracted mean $M_{1 / 2}$ and $Q_{\text {unif }}$ values can be used to determine the value of $M_{3}(Q=1 \mathrm{TeV})$ and consequently, the physical gluino mass. In this case, predictions for the expected value of $m_{\tilde{g}}$ may be made which can serve as a target for future hadron collider searches or compared to the mass of an already-discovered gluino.

\section{B. Running gaugino masses for ILC1}

The weak scale ILC1 parameters are sampled according to Gaussian distributions for $M_{1}$ and $M_{2}$ and uniformly

TABLE XXII. Relic density from MICROMEGAS and ASTROFIT, and direct and indirect detection cross sections from ASTROFIT in the pMSSM-10 fits to ILC1, ILC2, and nGMM1 observables (without the gluino mass measurement). $\Omega_{\text {Planck }}$ is taken to be 0.1199 [73].

\begin{tabular}{lccc}
\hline \hline Observable & ILC1 & ILC2 & nGMM1 \\
\hline$\sigma^{\text {SI }}$ model $\left[10^{-9} \mathrm{pb}\right]$ & 259.3 & 316.9 & 328.5 \\
$\sigma^{\mathrm{SI}}$ best fit $\left[10^{-9} \mathrm{pb}\right]$ & $260.7_{-6.9}^{+4.1}$ & $317.0_{-2.1}^{+2.1}$ & $328.5_{-0.9}^{+1.5}$ \\
$\langle\sigma v\rangle$ model $\left[10^{-27} \mathrm{~cm}^{3} \mathrm{~s}^{-1}\right]$ & 15.36 & 3.439 & 0.597 \\
$\langle\sigma v\rangle$ best fit $\left[10^{-27} \mathrm{~cm}^{3} \mathrm{~s}^{-1}\right]$ & $15.01_{-0.88}^{+1.52}$ & $3.501_{0.523}^{+5.741}$ & $0.621_{-0.165}^{+0.994}$ \\
\hline \hline
\end{tabular}

within the $1 \sigma$ range for $M_{3}$, motivated by the shape of the $\chi^{2}$ distributions obtained in the pMSSM-10 fits discussed in Sec. V B 1. The resulting running of the gaugino mass parameters in the ILC1 pMSSM-10 fit is plotted in Fig. 24(a). From the plot, it can be seen that $M_{1}$ and $M_{2}$ cross near $10^{16} \mathrm{GeV}$, which would verify the prediction of a SUSY GUT model. The uncertainty band for $M_{3}$ is quite wide but is consistent with the hypothesis of unification of all three gaugino masses at the same energy scale. The extracted unification scale $Q_{\text {unif }}$ for $M_{1}$ and $M_{2}$ is plotted in Fig. 25(a) from which it can be seen that the distribution follows a Gaussian. The gaugino mass unification scale is found to be $Q_{\text {unif }}=3.8 \times 10^{16} \mathrm{GeV}$ with a $68 \%$ confidence range of $\left[3.0 \times 10^{15}, 4.9 \times 10^{17}\right] \mathrm{GeV}$. From Fig. 25(b), the unified gaugino mass parameter is found to be $M_{1 / 2}=583 \pm 40 \mathrm{GeV}$ in agreement with the GUT scale model fit.

If it is then assumed that the unification is due to an NUHM2 model, and true model parameter values are assumed for parameters other than $M_{1 / 2}$, then instead $M_{3}$ can be extracted by running down in energy to find the running value of $M_{3}(Q=1 \mathrm{TeV})$. From Fig. 24(b), we obtain $M_{3}(Q=1 \mathrm{TeV})=1216 \pm 76 \mathrm{GeV}$ (which agrees with the weak scale fitted value). Consequently, a prediction for the physical gluino mass can be obtained: $m_{\tilde{g}}=1467 \pm 80 \mathrm{GeV}$, which could then be checked against results from hadron collider searches.

\section{Running gaugino masses for ILC2}

The uncertainties of the weak scale gaugino mass fit parameters are larger in the case of ILC2 as compared to ILC1. Still, the weak scale ILC2 parameters are sampled according to Gaussian distributions for $M_{1}$ and $M_{2}$ and uniformly within the $1 \sigma$ range for $M_{3}$, motivated by the shape of the $\chi^{2}$ distributions obtained in the pMSSM-10 fits discussed in Sec. VB 2. The larger uncertainties are reflected in the running gaugino mass plots in Figs. 26(a) and 26(b). Nevertheless, it is still possible to verify that $M_{1}$ and $M_{2}$ unify near the GUT scale. For ILC2, the fitted weak 


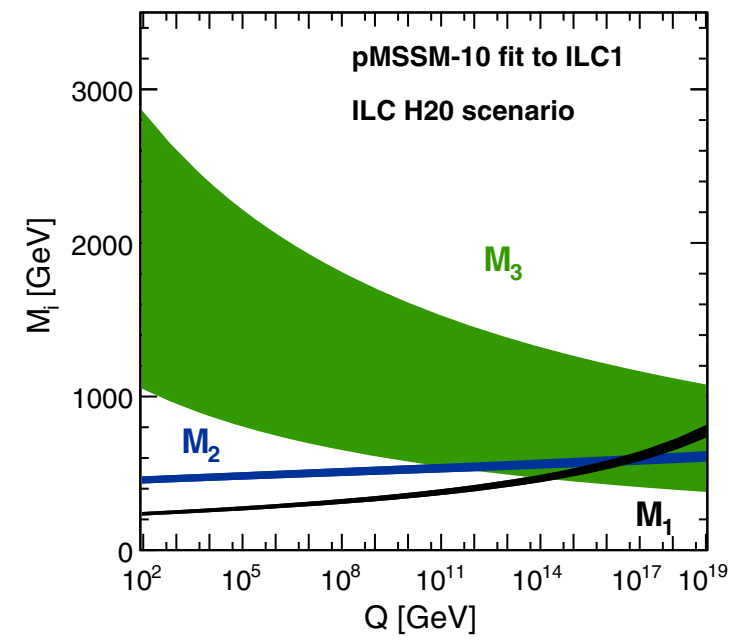

(a)

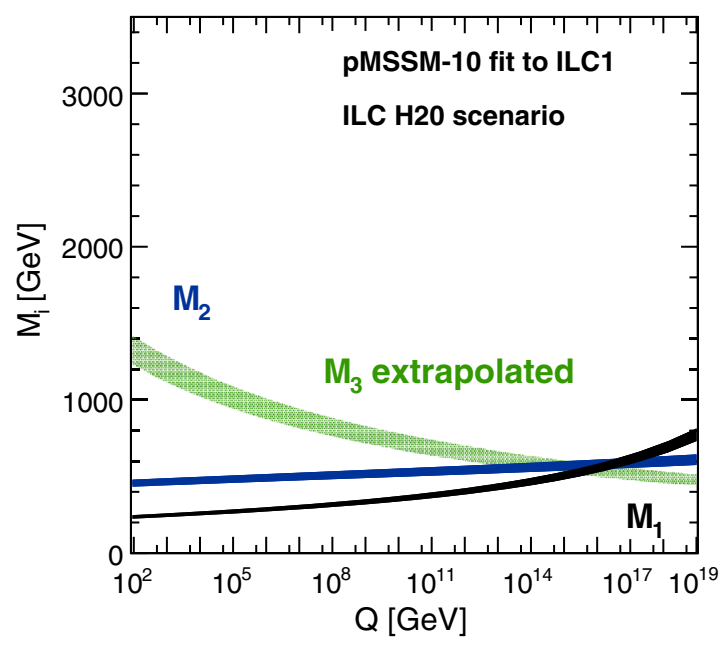

(b)

FIG. 24. The running gaugino masses $M_{i}$ based on the pMSSM-10 fit to ILC1 observables. The bands correspond to one standard deviation. (a) Using $M_{3}$ at the weak scale as constrained from ILC measurements (b) $M_{3}$ is assumed to unify with $M_{1}$ and $M_{2}$ and then run to the weak scale to gain a prediction for $M_{3}(Q=1 \mathrm{TeV})$.

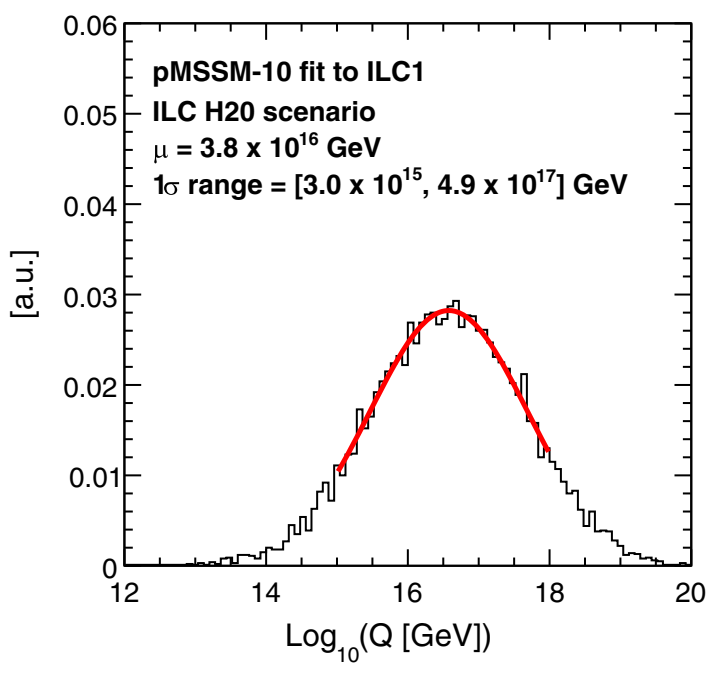

(a) Unification scale $Q_{\text {unif }}$

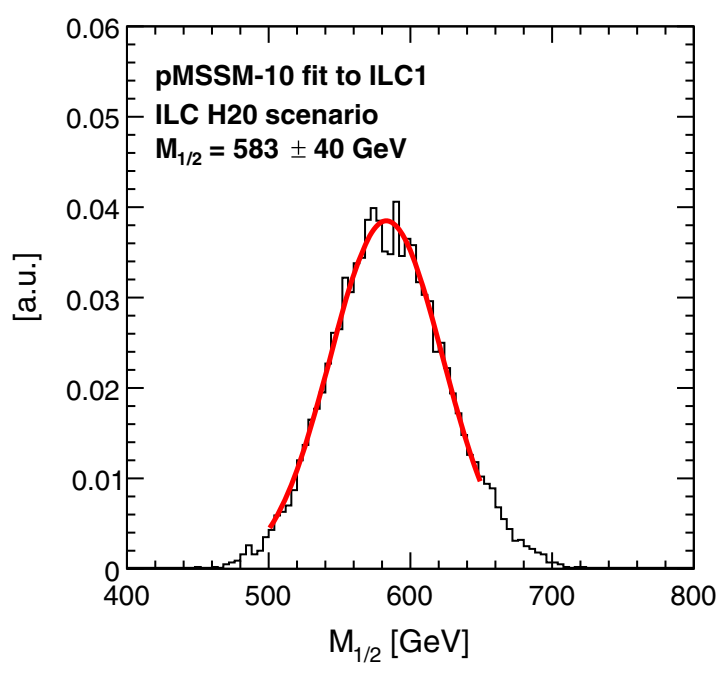

(b) unified gaugino mass $M_{1 / 2}$

FIG. 25. Distribution of the unification scale $Q_{\text {unif }}$ and unified gaugino mass $M_{1 / 2}$ obtained from the running parameters $M_{1}$ and $M_{2}$ and their uncertainties from the pMSSM-10 fit to ILC1 observables.

scale error band for $M_{3}$ is so wide that it is consistent with unification with $M_{1}$ or $M_{2}$ at almost any scale.

Using the same methodology as for ILC1, the unification scale for ILC2 where $M_{1}=M_{2}$ is found to be Gaussian with a mean of $Q_{\text {unif }}=1.5 \times 10^{16} \mathrm{GeV}$ with a $68 \%$ confidence interval of $\left[5.4 \times 10^{13}, 4.4 \times 10^{18}\right] \mathrm{GeV}$, as shown in Fig. 27(a). The unified value of $M_{1 / 2}$ is found in Fig. 27(b) to be Gaussian with $M_{1 / 2}=1220 \pm 170 \mathrm{GeV}$, which corresponds to the GUT scale fit model value. If $M_{3}$ is instead assumed to unify with $M_{1}$ and $M_{2}$ at $Q_{\text {unif }}$ and the NUHM2 model is adopted, then the extrapolated value of $M_{3}$ at $1 \mathrm{TeV}$ is found to be $M_{3}(Q=1 \mathrm{TeV})=2616 \pm$ $582 \mathrm{GeV}$ while the physical gluino mass is found to be $m_{\tilde{g}}=2872 \pm 605 \mathrm{GeV}$. Such a large value may serve as a target for gluino pair searches at upgraded hadron colliders.

\section{Running gaugino masses for nGMM1}

The running of the gaugino mass parameters in the nGMM1 benchmark model differs from the running in the ILC1 and ILC2 models. There are two reasons: 1. the underlying model is now a mirage unification model where the gaugino mass parameters unify at an intermediate energy scale, and 2. the determination of $M_{1}$ and $M_{2}$ from the weak scale fits is much less accurate in nGMM1 as compared to the ILC1 and ILC2 benchmark models. 


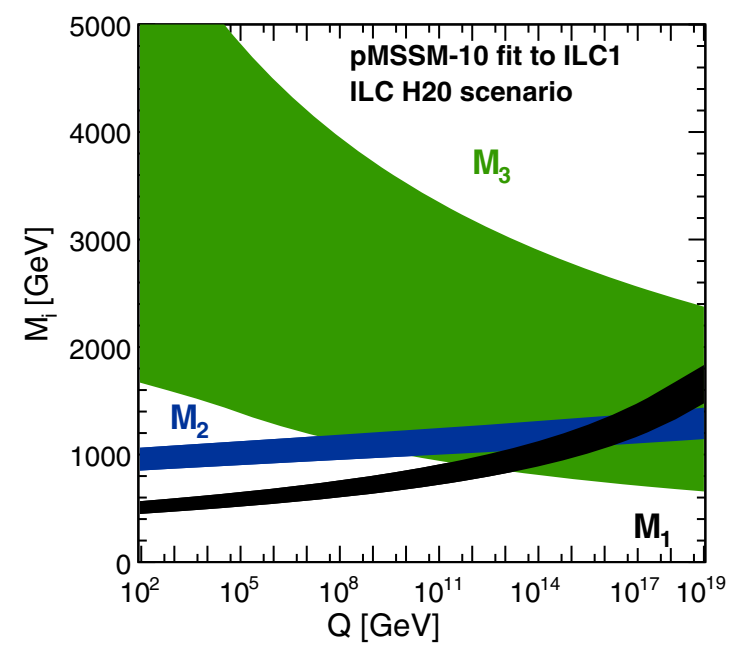

(a)

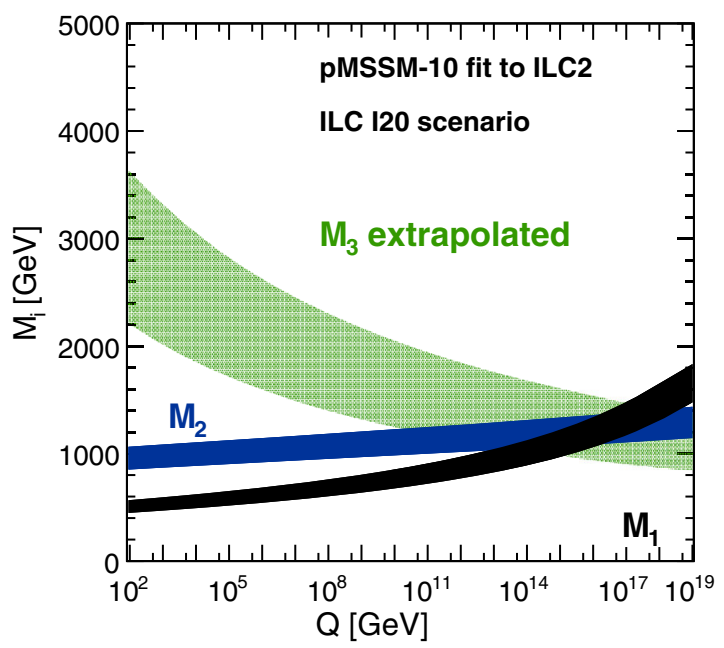

(b)

FIG. 26. The running gaugino masses $M_{i}$ based on the pMSSM-10 fit to ILC2 observables. The bands correspond to one standard deviation. (a) Using $M_{3}$ at the weak scale as constrained from ILC measurements (b) $M_{3}$ is assumed to unify with $M_{1}$ and $M_{2}$ and then run to the weak scale to gain a prediction for $M_{3}(Q=1 \mathrm{TeV})$.

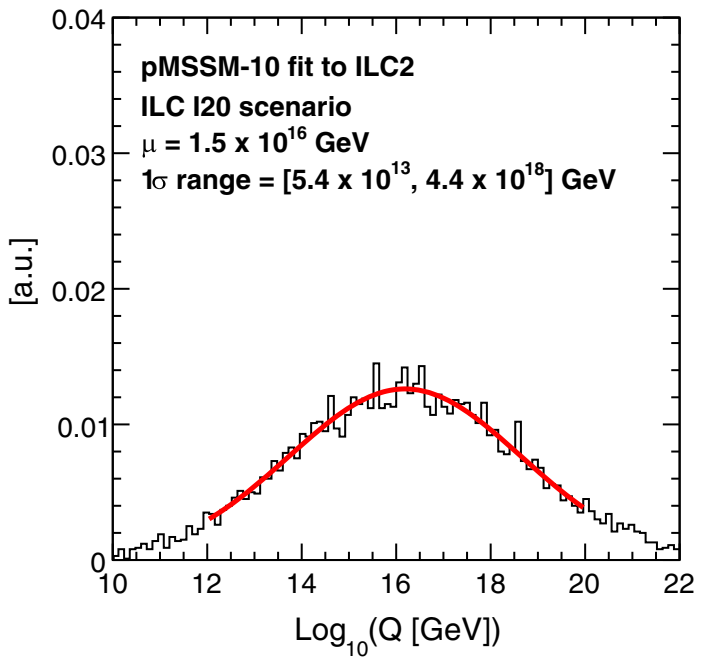

(a) Unification scale $Q_{\text {unif }}$

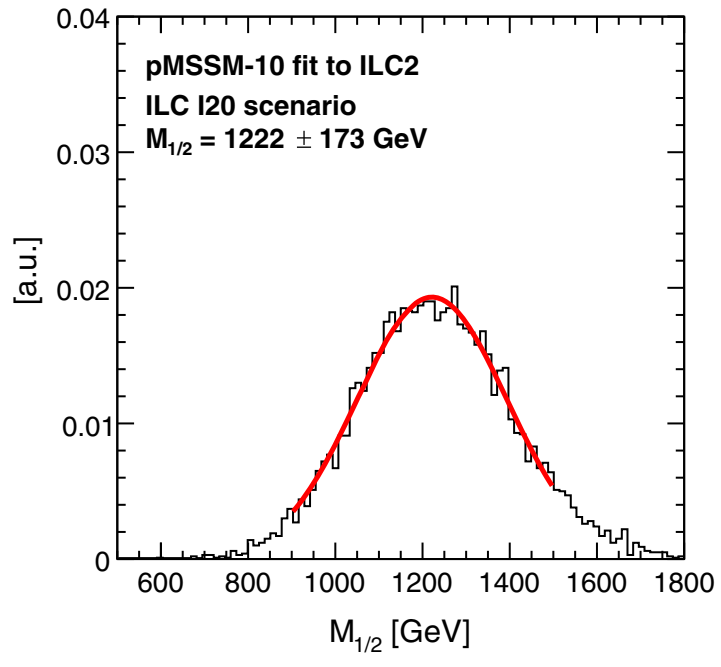

(b) unified gaugino mass $M_{1 / 2}$

FIG. 27. Distribution of the unification scale $Q_{\text {unif }}$ and unified gaugino mass $M_{1 / 2}$ obtained from the running parameters $M_{1}$ and $M_{2}$ and their uncertainties from the pMSSM-10 fit to ILC2 observables.

Figure 28(a) shows the running gaugino masses resultant from the pMSSM-10 fit with absolute masses as input as described in Sec. V B 3. Even in this most conservative case, the plot is certainly inconsistent with any sort of GUT scale unification of gaugino masses. From a closer look, we notice that the hierarchy between $M_{1}$ and $M_{2}$ at $Q=$ $1 \mathrm{TeV}$ is not well defined, and that actually the $M_{1}$ band seems to start above the lower rim of the $M_{2}$ band. This effect occurs since, motivated by the shape of the $\chi^{2}$ landscape of the pMSSM-10 fit (cf. Fig. 19), $M_{1}$ and $M_{3}$ are sampled from a uniform distribution and only $M_{2}$ is treated with a Gaussian. In addition, the $1 \sigma$ interval for $M_{1}$ is very asymmetric around the best fit point (cf. Table XX). In combination with the flat sampling, the $1 \sigma$ band for $M_{1}$ seems to start much higher than the best fit value for $M_{1}$ would indicate.

A substantial improvement of the precision can be seen in Fig. 28(b), which shows the analogous result obtained when using the improved experimental precisions presented in Sec. IV C plus a 10\% measurement of the gluino mass from the HL-LHC (or other future hadron collider). In addition to the improved inputs, the parameter extraction has also been refined: the estimates of $M_{1}, M_{2}$, and $M_{3}$ at the weak scale are obtained from a fit of only the pMSSM-4 


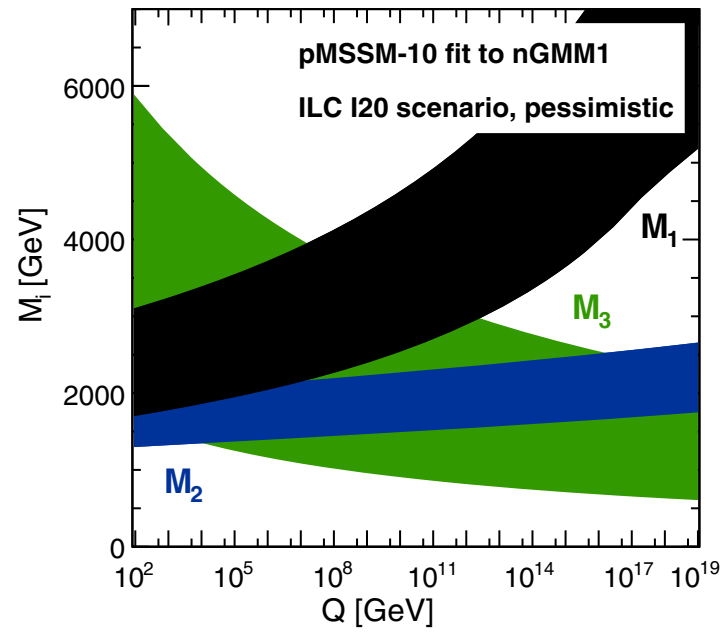

(a)

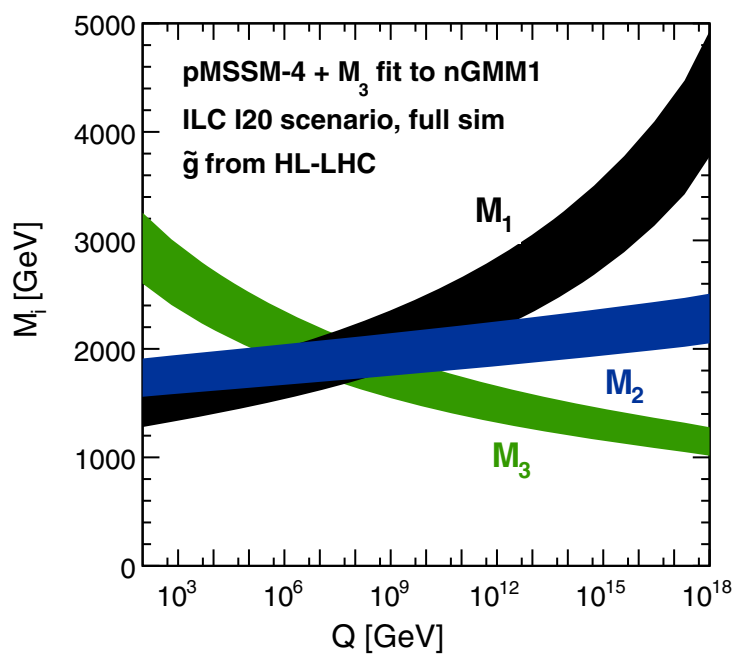

(b)

FIG. 28. The running of the gaugino masses after extracting their weak scale values from a fit to nGMM1 observables. The bands correspond to one standard deviation. (a) pMSSM-10 fit result with absolute masses as input from Sec. V B 3 (b) estimated effect of improvement from using the full simulation results from Sec. IV C, and from including a 10\% measurement of the gluino mass from HL-LHC (or other future hadron collider). In addition a fit of the pMSSM-4 parameters, and $M_{3}$ as a fit parameter, is run, as discussed at the beginning of Sec. V B. The Markov chain had a length of $10^{5}$ points.

parameters and $M_{3}$, which could be run subsequently to an initial pMSSM-10 fit as outlined at the beginning of Sec. V B. In this case, all parameters can be sampled from Gaussian distributions, as can be seen from Fig. 19. The weak scale hierarchy between $M_{1}$ and $M_{2}$ is now well determined, and a clear crossing of all three bands is found at a scale much lower than the GUT scale: around $10^{7}-10^{8} \mathrm{GeV}$, consistent with the theory mass unification scale for the model point which occurs at $10^{7} \mathrm{GeV}$.

This is not even the most optimistic case, since further improvements can be expected from using the Higgsino mass differences instead as input [cf. Fig. 21(d)] and from more precise $\tilde{\chi}_{1}^{ \pm}$and $\tilde{\chi}_{2}^{0}$ masses extracted from scanning the thresholds of $\tilde{\chi}_{1}^{+} \tilde{\chi}_{1}^{-}$and $\tilde{\chi}_{2}^{0} \tilde{\chi}_{i}^{0}$ production, respectively. In addition, the consideration of further constraints from improved EWPOs, flavor physics, direct search limits etc., is expected to further improve the weak scale parameter determination.

For the conservative version of the running masses in Fig. 28(a), we quantify the constraints on the unification scale in Fig. 29(a). While the distribution of obtained $Q_{\text {unif }}$ values has no clear peak, it increases towards lower

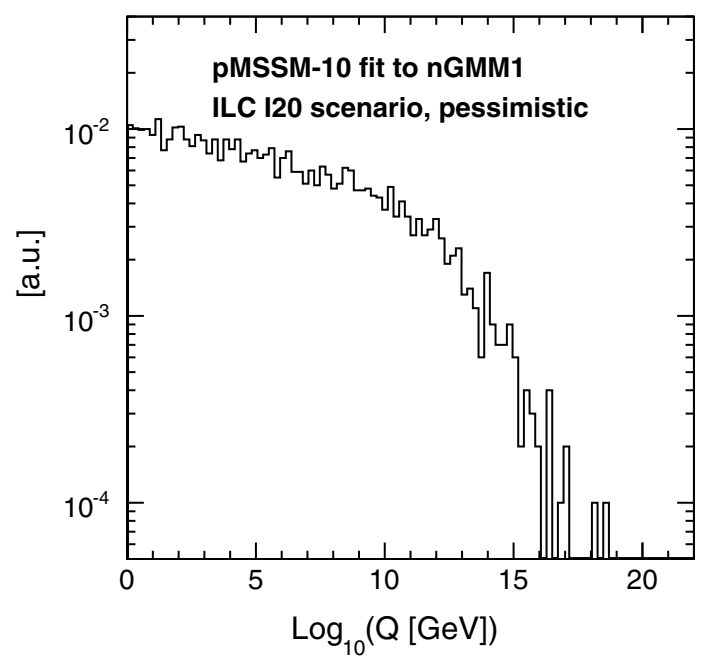

(a) Unification scale $Q_{\text {unif }}$

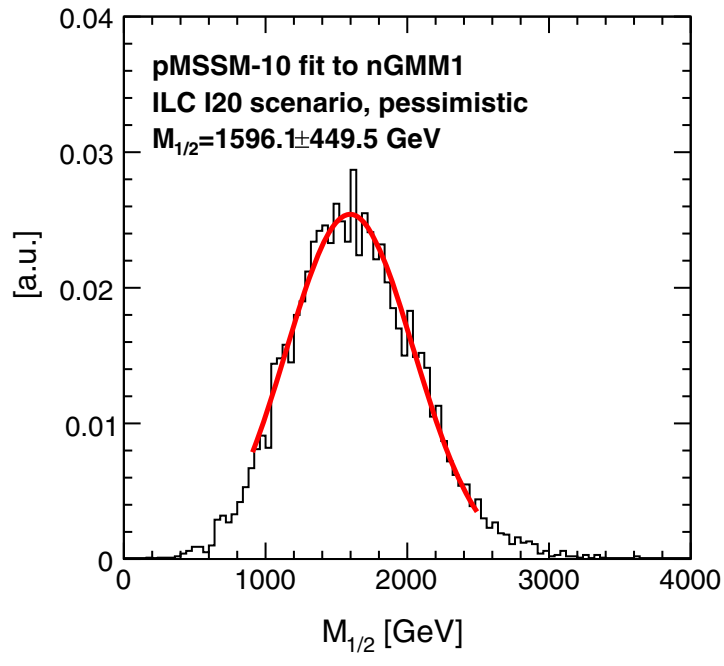

(b) unified gaugino mass $M_{1 / 2}$

FIG. 29. Distribution of the unification scale $Q_{\text {unif }}$ and unified gaugino mass $M_{1 / 2}$ obtained from the running parameters $M_{1}$ and $M_{2}$ and their uncertainties from the pMSSM-10 fit to nGMM1 observables. 
unification scales, away from the GUT scale. A unification at $\sim 10^{16} \mathrm{GeV}$ is excluded with $99.9 \%$ probability. The most probable unified value of $M_{1 / 2}$ was found to be $1600 \pm 450 \mathrm{GeV}$ in Fig. 29(b). The drastic improvement in Fig. 28(b) compared to Fig. 28, illustrates the substantial impact which can be expected from further refinements of the underlying analysis.

Due to the extracted gaugino mass unification scale not matching with the GUT scale, there would be important implications for SUSY model building. It is noteworthy that the pMSSM fit and the fit parameter evolution indicate that the underlying model does not have gaugino mass unification, even though the fit of NUHM2 parameters to the nGMM1 observables presented in Sec. VA does not entirely rule NUHM2 out as a possible model.

\section{SUMMARY AND CONCLUSIONS}

Supersymmetry with radiatively driven naturalness is especially compelling in that it reconciles electroweak naturalness with (multi-TeV) LHC sparticle mass limits and Higgs boson mass measurements. The most fundamental consequence of radiatively driven natural SUSY is the prediction of four light Higgsinos $\chi_{1}^{ \pm}, \chi_{1,2}^{0}$ with a mass $\sim 100-300 \mathrm{GeV}$ (the lower the better). Such light Higgsinos are difficult (but perhaps not impossible) to see at LHC, but would be easily visible at ILC operating with $\sqrt{s}>2 m$ (Higgsino). In this case, the ILC, initially constructed as a Higgs factory, would turn out to be a Higgsino factory. Thus, for this highly motivated scenario, ILC could serve as both a SUSY discovery (or confirmation) machine, and a precision microscope.

In this paper, we have examined the capability of experiments at the ILC to both discover (or confirm) supersymmetry and to make precision measurements of superparticle properties that would probe the superpotential Higgsino mass parameter $\mu$ via direct sparticle mass measurements and, in addition, provide a measurement of SUSY-breaking gaugino mass parameters via the Higgsino mass splittings.

When these measurements are combined with precision Higgs boson measurements, precision fits to both weak scale SUSY and high scale SUSY model parameters can be made. We have investigated the capability of ILC to discover light Higgsinos in three natural SUSY benchmark models: two with unified gaugino masses and one with mirage unification of gaugino masses at an intermediate mass scale between $m_{\mathrm{GUT}}$ and $m_{\text {weak }}$. Our calculations implement a detailed ILD detector simulation along with an event generation from WHIZARD.

By measuring $e^{+} e^{-} \rightarrow \tilde{\chi}_{1}^{+} \tilde{\chi}_{1}^{-} \rightarrow\left(\ell \nu_{\ell} \tilde{\chi}_{1}^{0}\right)+\left(q \bar{q}^{\prime} \tilde{\chi}_{1}^{0}\right)$, we are able to extract $m_{\tilde{\chi}_{1}^{ \pm}}$and $m_{\tilde{\chi}_{1}^{0}}$ via the $m(j j)$ and $E(j j)$ distributions, typically to a percent level accuracy. By measuring the dilepton mass and energy distributions from $e^{+} e^{-} \rightarrow \tilde{\chi}_{2}^{0} \tilde{\chi}_{1}^{0}$ followed by $\tilde{\chi}_{2}^{0} \rightarrow \ell^{+} \ell^{-} \tilde{\chi}_{1}^{0}$, we are able to measure $m_{\tilde{\chi}_{1}^{0}}$ and $m_{\tilde{\chi}_{2}^{0}}$ to typically a percent level accuracy. We combine the Higgsino mass measurements with precision Higgsino pair production cross section measurements using different beam polarizations.

When these precision Higgsino measurements are combined with precision Higgs boson measurements, precision fits to both weak scale SUSY and high scale SUSY model parameters can be made. In particular, an indirect measurement of wino and bino SUSY breaking masses can be extracted from the Higgsino mass splittings. When extrapolated to high energies, the hypothesis of gaugino mass unification can be tested. If combined with LHC gluino mass measurements, the unification of all three gaugino masses may be explored. Such measurements will shed light on different possibilities for SUSY breaking as may be expected in SUSY GUT models or in models with mixed moduli and anomaly (mirage) mediation. In addition, fits of SUSY dark matter observables may shed light on the nature of dark matter, such as confirming or ruling out multicomponent dark matter as expected from natural SUSY where both Higgsino-like WIMPs and axions are expected to be produced in the early Universe.

Thus, in assessing the ILC capabilities in this compelling SUSY extension of the SM, we conclude that ILC can indeed serve as a SUSY discovery machine and precision microscope, offering a window into the intricacies of SUSY breaking and fundamental particle physics and providing insights into the nature of dark matter and cosmology.

\section{ACKNOWLEDGMENTS}

This work was carried out in the context of the ILD detector concept group. We would like to thank the LCC generator working group and the ILD software working group for providing the simulation and reconstruction tools and producing the Monte Carlo samples used in this study. This work was supported in part by the Office of Science, US Department of Energy, the Japan Society for the Promotion of Science (JSPS) under Grants in-Aid for Science Research 16H02173 and 16H02176, and by the Deutsche Forschungsgemeinschaft (DFG) through the Collaborative Research Centre SFB 676 "Particles, Strings and the Early Universe", project B1. This work has benefited from computing services provided by the ILC Virtual Organization, supported by the national resource providers of the EGI Federation and the Open Science GRID, and of those of the German National Analysis Facility (NAF). 


\section{APPENDIX: ADDITIONAL FIGURES AND TABLES}

TABLE XXIII. Expected number of events for chargino signal and major backgrounds for the electron final state and beam polarization $\mathcal{P}_{-+}$. The integrated luminosity is assumed to be $500 \mathrm{fb}^{-1}$. For each benchmark model, the background refers to the other SUSY backgrounds.

\begin{tabular}{|c|c|c|c|c|c|c|c|c|c|c|c|}
\hline \multirow{2}{*}{$\begin{array}{l}\tilde{\chi}_{1}^{+} \tilde{\chi}_{1}^{-} \rightarrow \tilde{\chi}_{1}^{0} \tilde{\chi}_{1}^{0} q \bar{q}^{\prime} e \nu_{e} \\
500 \mathrm{GeV}, 500 \mathrm{fb}^{-1}, \mathcal{P}_{-+}\end{array}$} & \multicolumn{2}{|c|}{ ILC1 } & \multicolumn{2}{|c|}{ ILC2 } & \multicolumn{2}{|c|}{ nGMM1 } & \multicolumn{5}{|c|}{ SM bkg. } \\
\hline & Signal & Bkg. & Signal & Bkg. & Signal & Bkg. & $e^{+} e^{-} \rightarrow 2 \mathrm{f}$ & $e^{+} e^{-} \rightarrow 4 \mathrm{f}$ & $\gamma \gamma \rightarrow 2 \mathrm{f}$ & $e \gamma \rightarrow 3 \mathrm{f}$ & $\gamma \gamma \rightarrow 4 \mathrm{f}$ \\
\hline Preselection & 53963 & 423992 & 41962 & 322011 & 66118 & 476646 & 11906936 & 14941264 & 307189572 & 65344394 & 61765 \\
\hline Lepton selection & 4926 & 11922 & 2733 & 7676 & 4453 & 12325 & 543911 & 914027 & 93465142 & 21607557 & 1905 \\
\hline BeamCal veto & 4869 & 11752 & 2707 & 7602 & 4414 & 12188 & 495890 & 748137 & 1284355 & 3964924 & 1772 \\
\hline$p_{T}>5 \mathrm{GeV}$ & 3146 & 2323 & 1242 & 1110 & 1337 & 1109 & 226624 & 506571 & 967020 & 3804929 & 1328 \\
\hline$N_{\text {trk,jet }} \geq 2$ & 2285 & 324 & 667 & 108 & 515 & 98 & 42892 & 220378 & 65284 & 1745715 & 627 \\
\hline$\left|\cos \theta_{j}\right|<0.98$ & 2225 & 314 & 652 & 106 & 504 & 97 & 15612 & 168407 & 50786 & 1323463 & 513 \\
\hline$\Delta \phi<1.0$ & 1544 & 122 & 411 & 30 & 296 & 19 & 1507 & 34570 & 11157 & 533787 & 51 \\
\hline$\left|\cos \theta_{j j}\right|<0.2$ & 1535 & 90 & 405 & 24 & 293 & 17 & 1360 & 32195 & 9471 & 483002 & 40 \\
\hline$E_{\mathrm{vis}}<80$ & 1496 & 87 & 402 & 24 & 291 & 17 & 59 & 403 & 1810 & 7835 & 2.9 \\
\hline$E_{\text {miss }}>400$ & 1485 & 87 & 402 & 24 & 291 & 17 & 12 & 69 & 7.1 & 48 & 2.0 \\
\hline$\left|\cos \theta_{\text {miss }}\right|<0.99$ & 1463 & 85 & 392 & 23 & 283 & 15 & 5.9 & 64 & 0.0 & 22 & 2.0 \\
\hline
\end{tabular}

TABLE XXIV. Expected number of events for chargino signal and major backgrounds for the muon final state and beam polarization $\mathcal{P}_{-+}$. The integrated luminosity is assumed to be $500 \mathrm{fb}^{-1}$. For each benchmark model, the background refers to the other SUSY backgrounds.

\begin{tabular}{|c|c|c|c|c|c|c|c|c|c|c|c|}
\hline \multirow{2}{*}{$\begin{array}{l}\tilde{\chi}_{1}^{+} \tilde{\chi}_{1}^{-} \rightarrow \tilde{\chi}_{1}^{0} \tilde{\chi}_{1}^{0} q \bar{q}^{\prime} \mu \nu_{\mu} \\
500 \mathrm{GeV}, 500 \mathrm{fb}^{-1}, \mathcal{P}_{-+}\end{array}$} & \multicolumn{2}{|c|}{ ILC1 } & \multicolumn{2}{|c|}{ ILC2 } & \multicolumn{2}{|c|}{ nGMM1 } & \multicolumn{5}{|c|}{ SM bkg. } \\
\hline & Signal & Bkg. & Signal & Bkg. & Signal & Bkg. & $e^{+} e^{-} \rightarrow 2 \mathrm{f}$ & $e^{+} e^{-} \rightarrow 4 \mathrm{f}$ & $\gamma \gamma \rightarrow 2 \mathrm{f}$ & $e \gamma \rightarrow 3 \mathrm{f}$ & $\gamma \gamma \rightarrow 4 \mathrm{f}$ \\
\hline Preselection & 53459 & 424497 & 41714 & 322259 & 65104 & 477660 & 11906936 & 14941264 & 307189572 & 65344394 & 61765 \\
\hline Lepton selection & 5748 & 32945 & 3497 & 21394 & 6194 & 34867 & 1125893 & 1297965 & 42676970 & 2497567 & 2716 \\
\hline BeamCal veto & 5683 & 32500 & 3462 & 21165 & 6134 & 34476 & 1025945 & 1049378 & 420779 & 325406 & 2475 \\
\hline$p_{T}>5 \mathrm{GeV}$ & 3677 & 3141 & 1566 & 1720 & 1832 & 1794 & 99197 & 345356 & 101920 & 146861 & 1430 \\
\hline$N_{\text {trk,jet }} \geq 2$ & 2612 & 710 & 805 & 225 & 690 & 228 & 19319 & 183151 & 197 & 10945 & 509 \\
\hline$\left|\cos \theta_{j}\right|<0.98$ & 2544 & 688 & 784 & 221 & 672 & 223 & 11089 & 150507 & 28 & 7906 & 331 \\
\hline$\Delta \phi<1.0$ & 1972 & 259 & 532 & 53 & 412 & 40 & 755 & 37957 & 28 & 874 & 55 \\
\hline$\left|\cos \theta_{j j}\right|<0.2$ & 1954 & 118 & 526 & 29 & 406 & 32 & 471 & 37320 & 0.0 & 174 & 51 \\
\hline$E_{\mathrm{vis}}<80$ & 1905 & 110 & 523 & 28 & 404 & 31 & 59 & 379 & 0.0 & 0.0 & 22 \\
\hline$E_{\text {miss }}>400$ & 1889 & 110 & 523 & 28 & 404 & 31 & 33 & 39 & 0.0 & 0.0 & 8.0 \\
\hline$\left|\cos \theta_{\text {miss }}\right|<0.99$ & 1862 & 108 & 509 & 28 & 389 & 29 & 33 & 37 & 0.0 & 0.0 & 7.0 \\
\hline
\end{tabular}

TABLE XXV. Expected number of events for chargino signal and major backgrounds for the electron final state and beam polarization $\mathcal{P}_{+-}$. The integrated luminosity is assumed to be $500 \mathrm{fb}^{-1}$. For each benchmark model, the background refers to the other SUSY backgrounds.

\begin{tabular}{|c|c|c|c|c|c|c|c|c|c|c|c|}
\hline \multirow{2}{*}{$\begin{array}{l}\tilde{\chi}_{1}^{+} \tilde{\chi}_{1}^{-} \rightarrow \tilde{\chi}_{1}^{0} \tilde{\chi}_{1}^{0} q \bar{q}^{\prime} e \nu_{e} \\
500 \mathrm{GeV}, 500 \mathrm{fb}^{-1}, \mathcal{P}_{+-}\end{array}$} & \multicolumn{2}{|c|}{ ILC1 } & \multicolumn{2}{|c|}{ ILC2 } & \multicolumn{2}{|c|}{ nGMM1 } & \multicolumn{5}{|c|}{ SM bkg. } \\
\hline & Signal & Bkg. & Signal & Bkg. & Signal & Bkg. & $e^{+} e^{-} \rightarrow 2 \mathrm{f}$ & $e^{+} e^{-} \rightarrow 4 \mathrm{f}$ & $\gamma \gamma \rightarrow 2 \mathrm{f}$ & $e \gamma \rightarrow 3 f$ & $\gamma \gamma \rightarrow 4 \mathrm{f}$ \\
\hline Preselection & 13276 & 163541 & 10382 & 97217 & 17159 & 180558 & 7839612 & 4800015 & 307189572 & 64002532 & 61765 \\
\hline Lepton selection & 1251 & 5394 & 660 & 2546 & 1153 & 5528 & 434539 & 449786 & 93465142 & 20689292 & 1905 \\
\hline BeamCal veto & 1238 & 5320 & 653 & 2518 & 1143 & 5467 & 395552 & 293541 & 1284355 & 3493039 & 1772 \\
\hline$p_{T}>5 \mathrm{GeV}$ & 834 & 787 & 298 & 310 & 350 & 323 & 210050 & 199506 & 967020 & 3341264 & 1328 \\
\hline$N_{\text {trk,jet }} \geq 2$ & 615 & 116 & 161 & 30 & 140 & 27 & 39447 & 50256 & 65284 & 1297669 & 627 \\
\hline$\left|\cos \theta_{j}\right|<0.98$ & 600 & 113 & 157 & 29 & 137 & 26 & 13665 & 24867 & 50786 & 900538 & 513 \\
\hline$\Delta \phi<1.0$ & 423 & 47 & 100 & 6.9 & 77 & 6.1 & 1376 & 4950 & 11157 & 386411 & 51 \\
\hline$\left|\cos \theta_{j j}\right|<0.2$ & 421 & 25 & 99 & 5.1 & 76 & 5.2 & 1275 & 4411 & 9471 & 358781 & 40 \\
\hline$E_{\mathrm{vis}}<80$ & 409 & 24 & 98 & 5.1 & 76 & 5.2 & 29 & 59 & 1810 & 7315 & 2.9 \\
\hline$E_{\text {miss }}>400$ & 408 & 24 & 98 & 5.1 & 76 & 5.2 & 7.8 & 19 & 7.1 & 22 & 2.0 \\
\hline$\left|\cos \theta_{\text {miss }}\right|<0.99$ & 404 & 23 & 96 & 4.6 & 73 & 5.1 & 7.4 & 16 & 0.0 & 8.0 & 2.0 \\
\hline
\end{tabular}


TABLE XXVI. Expected number of events for chargino signal and major backgrounds for the muon final state and beam polarization $\mathcal{P}_{+-}$. The integrated luminosity is assumed to be $500 \mathrm{fb}^{-1}$. For each benchmark model, the background refers to the other SUSY backgrounds.

\begin{tabular}{|c|c|c|c|c|c|c|c|c|c|c|c|}
\hline \multirow{2}{*}{$\begin{array}{l}\tilde{\chi}_{1}^{+} \tilde{\chi}_{1}^{-} \rightarrow \tilde{\chi}_{1}^{0} \tilde{\chi}_{1}^{0} q \bar{q}^{\prime} \mu \nu_{\mu} \\
500 \mathrm{GeV}, 500 \mathrm{fb}^{-1}, \mathcal{P}_{+-}\end{array}$} & \multicolumn{2}{|c|}{ ILC1 } & \multicolumn{2}{|c|}{ ILC2 } & \multicolumn{2}{|c|}{ nGMM1 } & \multicolumn{5}{|c|}{ SM bkg. } \\
\hline & Signal & Bkg. & Signal & Bkg. & Signal & Bkg. & $e^{+} e^{-} \rightarrow 2 \mathrm{f}$ & $e^{+} e^{-} \rightarrow 4 \mathrm{f}$ & $\gamma \gamma \rightarrow 2 \mathrm{f}$ & $e \gamma \rightarrow 3 \mathrm{f}$ & $\gamma \gamma \rightarrow 4 \mathrm{f}$ \\
\hline Preselection & 13222 & 163594 & 10352 & 97248 & 16876 & 180842 & 7839612 & 4800015 & 307189572 & 64002532 & 61765 \\
\hline Lepton selection & 1502 & 16551 & 869 & 7667 & 1619 & 18398 & 783612 & 536319 & 42676970 & 2358203 & 2716 \\
\hline BeamCal veto & 1487 & 16318 & 860 & 7574 & 1604 & 18183 & 710583 & 302815 & 420779 & 307086 & 2475 \\
\hline$p_{T}>5 \mathrm{GeV}$ & 993 & 1108 & 388 & 521 & 480 & 613 & 76202 & 72875 & 101920 & 140719 & 1430 \\
\hline$N_{\text {trk,jet }} \geq 2$ & 733 & 276 & 199 & 69 & 182 & 65 & 14401 & 18374 & 197 & 6910 & 509 \\
\hline$\left|\cos \theta_{j}\right|<0.98$ & 712 & 270 & 194 & 67 & 175 & 64 & 8258 & 11680 & 28 & 4533 & 331 \\
\hline$\Delta \phi<1.0$ & 555 & 134 & 134 & 20 & 107 & 10 & 622 & 3238 & 28 & 343 & 55 \\
\hline$\left|\cos \theta_{j j}\right|<0.2$ & 550 & 37 & 132 & 9.1 & 106 & 8.6 & 373 & 3093 & 0.0 & 66 & 51 \\
\hline$E_{\text {vis }}<80$ & 536 & 34 & 131 & 8.7 & 105 & 8.3 & 29 & 38 & 0.0 & 0.0 & 22 \\
\hline$E_{\text {miss }}>400$ & 532 & 34 & 131 & 8.7 & 105 & 8.3 & 8.2 & 9.5 & 0.0 & 0.0 & 8.0 \\
\hline$\left|\cos \theta_{\text {miss }}\right|<0.99$ & 524 & 34 & 127 & 8.5 & 101 & 8.2 & 8.2 & 7.2 & 0.0 & 0.0 & 7.0 \\
\hline
\end{tabular}

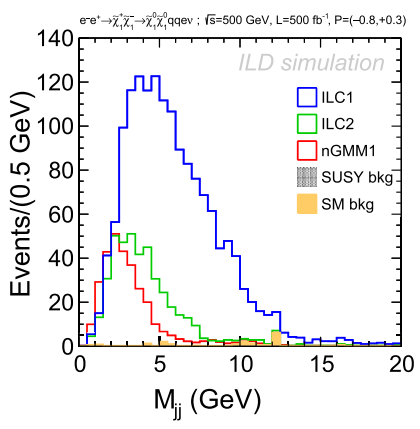

(a) $\tilde{\chi}_{1}^{0} \tilde{\chi}_{1}^{0} q \bar{q}^{\prime} e v_{e} ; \mathcal{P}_{-+}$

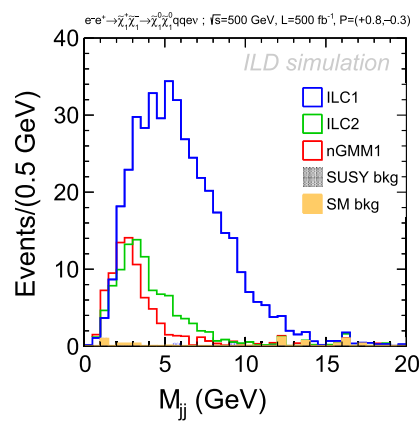

(b) $\tilde{\chi}_{1}^{0} \tilde{\chi}_{1}^{0} q \bar{q}^{\prime} e v_{e} ; \mathcal{P}_{+-}$

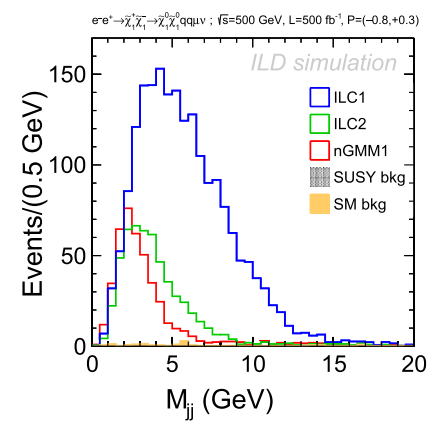

(c) $\tilde{\chi}_{1}^{0} \tilde{\chi}_{1}^{0} q \bar{q}^{\prime} \mu v_{\mu} ; \mathcal{P}_{-+}$

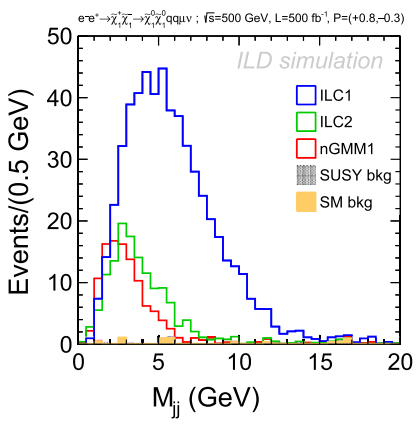

(d) $\tilde{\chi}_{1}^{0} \tilde{\chi}_{1}^{0} q \bar{q}^{\prime} \mu v_{\mu} ; \mathcal{P}_{+-}$

FIG. 30. Reconstructed dijet mass in the chargino channel $e^{+} e^{-} \rightarrow \tilde{\chi}_{1}^{+} \tilde{\chi}_{1}^{-} \rightarrow \tilde{\chi}_{1}^{0} \tilde{\chi}_{1}^{0} q \bar{q}^{\prime} \ell \nu_{\ell}$ for $500 \mathrm{fb}{ }^{-1}$ at $\sqrt{s}=500 \mathrm{GeV}$. In all cases, the background contributions are very small. The signal histograms are stacked on top of the backgrounds.

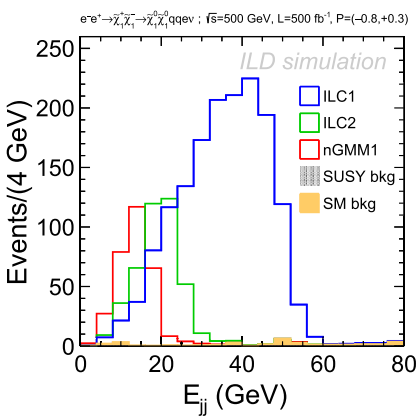

(a) $\tilde{\chi}_{1}^{0} \tilde{\chi}_{1}^{0} q \bar{q}^{\prime} e v_{e} ; \mathcal{P}_{-+}$

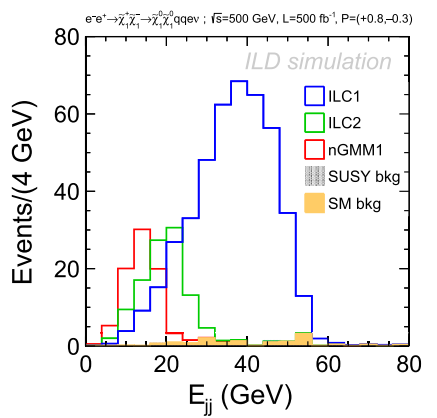

(b) $\tilde{\chi}_{1}^{0} \tilde{\chi}_{1}^{0} q \bar{q}^{\prime} e v_{e} ; \mathcal{P}_{+-}$

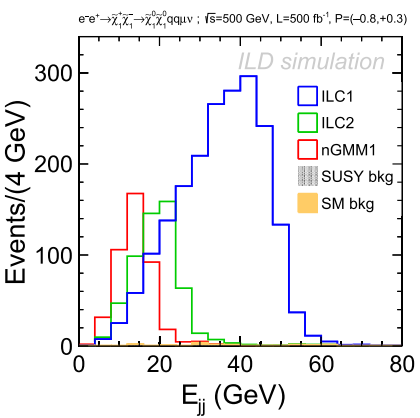

(c) $\tilde{\chi}_{1}^{0} \tilde{\chi}_{1}^{0} q \bar{q}^{\prime} \mu v_{\mu} ; \mathcal{P}_{-+}$

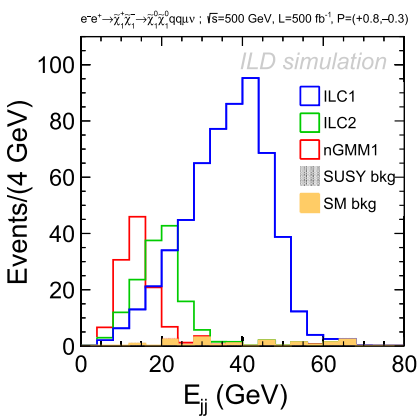

(d) $\tilde{\chi}_{1}^{0} \tilde{\chi}_{1}^{0} q \bar{q}^{\prime} \mu v_{\mu} ; \mathcal{P}_{+-}$

FIG. 31. Reconstructed dijet energy in the chargino channel $e^{+} e^{-} \rightarrow \tilde{\chi}_{1}^{+} \tilde{\chi}_{1}^{-} \rightarrow \tilde{\chi}_{1}^{0} \tilde{\chi}_{1}^{0} q \bar{q}^{\prime} \ell \nu_{\ell}$ for $500 \mathrm{fb}-1$ at $\sqrt{s}=500 \mathrm{GeV}$. In all cases, the background contributions are very small. The signal histograms are stacked on top of the backgrounds. 


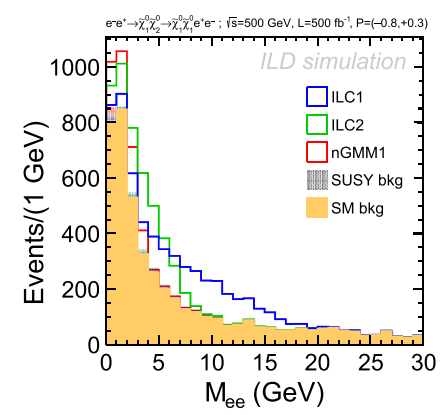

(a) $\tilde{\chi}_{1}^{0} \tilde{\chi}_{1}^{0} e^{+} e^{-} ; \mathcal{P}_{-+}$

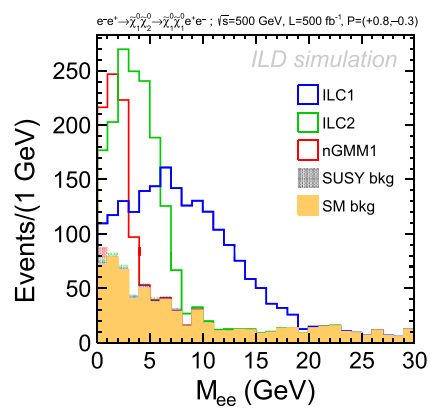

(b) $\tilde{\chi}_{1}^{0} \tilde{\chi}_{1}^{0} e^{+} e^{-} ; \mathcal{P}_{+-}$

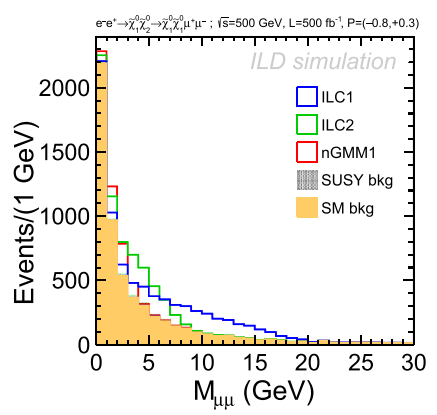

(c) $\tilde{\chi}_{1}^{0} \tilde{\chi}_{1}^{0} \mu^{+} \mu^{-} ; \mathcal{P}_{-+}$

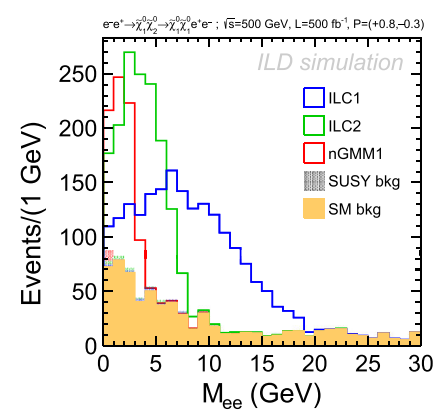

(d) $\tilde{\chi}_{1}^{0} \tilde{\chi}_{1}^{0} \mu^{+} \mu^{-} ; \mathcal{P}_{+-}$

FIG. 32. Reconstructed dilepton mass in the neutralino channel $e^{+} e^{-} \rightarrow \tilde{\chi}_{2}^{0} \tilde{\chi}_{1}^{0} \rightarrow \tilde{\chi}_{1}^{0} \tilde{\chi}_{1}^{0} \ell^{+} \ell^{-}$for $500 \mathrm{fb}^{-1}$ at $\sqrt{s}=500 \mathrm{GeV}$. In all cases, the SUSY background contributions are very small. The signal histograms are stacked on top of the backgrounds.

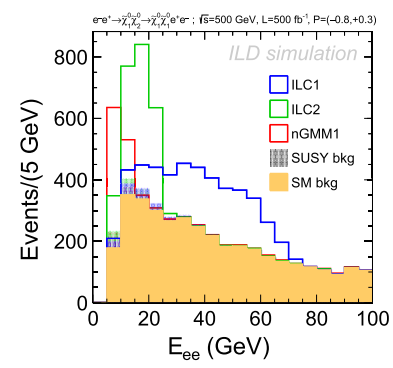

(a) $\tilde{\chi}_{1}^{0} \tilde{\chi}_{1}^{0} e^{+} e^{-} ; \mathcal{P}_{-+}$

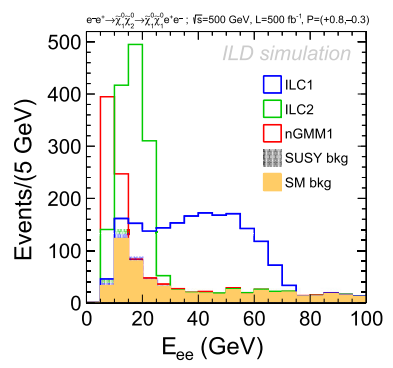

(b) $\tilde{\chi}_{1}^{0} \tilde{\chi}_{1}^{0} e^{+} e^{-} ; \mathcal{P}_{+-}$

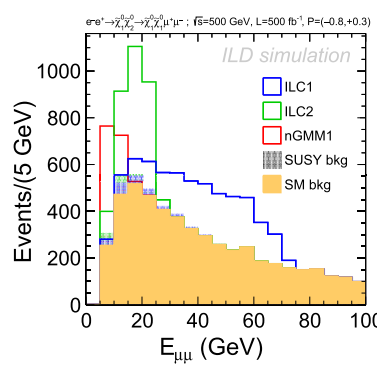

(c) $\tilde{\chi}_{1}^{0} \tilde{\chi}_{1}^{0} \mu^{+} \mu^{-} ; \mathcal{P}_{-+}$

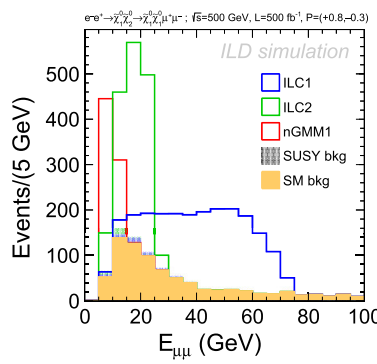

(d) $\tilde{\chi}_{1}^{0} \tilde{\chi}_{1}^{0} \mu^{+} \mu^{-} ; \mathcal{P}_{+-}$

FIG. 33. Reconstructed dilepton energy in the neutralino channel $e^{+} e^{-} \rightarrow \tilde{\chi}_{2}^{0} \tilde{\chi}_{1}^{0} \rightarrow \tilde{\chi}_{1}^{0} \tilde{\chi}_{1}^{0} \ell^{+} \ell^{-}$for $500 \mathrm{fb}^{-1}$ at $\sqrt{s}=500 \mathrm{GeV}$. In all cases, the SUSY background contributions are very small. The signal histograms are stacked on top of the backgrounds.

TABLE XXVII. Expected number of events for neutralino signal and major backgrounds for the electron final state and beam polarizations $\mathcal{P}\left(e^{-}, e^{+}\right)=(-0.8,+0.3)$. The integrated luminosity is assumed to be $500 \mathrm{fb}^{-1}$. For each benchmark model, the background refers to the other SUSY backgrounds.

\begin{tabular}{|c|c|c|c|c|c|c|c|c|c|c|c|}
\hline \multirow{2}{*}{$\begin{array}{l}\tilde{\chi}_{1}^{0} \tilde{\chi}_{2}^{0}\left(e^{-} e^{+}\right) \\
\mathcal{P}\left(e^{-}, e^{+}\right)=(-0.8,+0.3) \\
\end{array}$} & \multicolumn{2}{|c|}{ ILC1 } & \multicolumn{2}{|c|}{ ILC2 } & \multicolumn{2}{|c|}{ nGMM1 } & \multicolumn{5}{|c|}{ SM bkg. } \\
\hline & Sig. & Bkg. & Sig. & Bkg. & Sig. & Bkg. & $e^{+} e^{-} \rightarrow 2 \mathrm{f}$ & $e^{+} e^{-} \rightarrow 4 \mathrm{f}$ & $\gamma \gamma \rightarrow 2 \mathrm{f}$ & $e \gamma \rightarrow 3 \mathrm{f}$ & $\gamma \gamma \rightarrow 4 \mathrm{f}$ \\
\hline Preselection & 4370 & 15977 & 3098 & 12393 & 1076 & 8301 & 261999 & 1115296 & 87581 & 313496 & 14260 \\
\hline Lepton selection, $N_{\text {trk }}=2$ & 4028 & 4039 & 2866 & 3576 & 994 & 2837 & 23958 & 365653 & 22592 & 41791 & 192 \\
\hline BeamCal veto & 3965 & 3977 & 2831 & 3531 & 986 & 2808 & 18100 & 152375 & 22592 & 40935 & 176 \\
\hline$p_{T}>2.3 \mathrm{GeV}$ & 3822 & 3638 & 2504 & 3106 & 728 & 2329 & 16543 & 141410 & 21961 & 38709 & 103 \\
\hline$\left|\cos \theta_{\ell}\right|<0.95$ & 3601 & 3443 & 2455 & 2997 & 727 & 2257 & 9108 & 75460 & 21885 & 37151 & 82 \\
\hline$\Delta \phi<0.8$ & 2384 & 327 & 1696 & 371 & 653 & 365 & 3809 & 32251 & 14662 & 28218 & 34 \\
\hline$E_{\mathrm{vis}}<25 \mathrm{GeV}$ & 1621 & 189 & 1253 & 235 & 492 & 239 & 14 & 4768 & 15 & 669 & 25 \\
\hline$E_{\text {miss }}>300 \mathrm{GeV}$ & 1621 & 189 & 1253 & 235 & 492 & 239 & 14 & 4034 & 15 & 489 & 25 \\
\hline$\left|\cos \theta_{\text {miss }}\right|<0.98$ & 1621 & 189 & 1251 & 232 & 491 & 238 & 14 & 4021 & 14 & 488 & 25 \\
\hline$M_{\ell \ell}$ selection & 1621 & 185 & 1250 & 226 & 490 & 207 & 14 & 3875 & 14 & 371 & 19 \\
\hline
\end{tabular}


TABLE XXVIII. Expected number of events for neutralino signal and major backgrounds for the muon final state and beam polarizations $\mathcal{P}\left(e^{-}, e^{+}\right)=(-0.8,+0.3)$. The integrated luminosity is assumed to be $500 \mathrm{fb}^{-1}$. For each benchmark model, the background refers to the other SUSY backgrounds.

\begin{tabular}{|c|c|c|c|c|c|c|c|c|c|c|c|}
\hline \multirow{2}{*}{$\begin{array}{l}\tilde{\chi}_{1}^{0} \tilde{\chi}_{2}^{0}\left(\mu^{-} \mu^{+}\right) \\
\mathcal{P}\left(e^{-}, e^{+}\right)=(-0.8,+0.3) \\
\end{array}$} & \multicolumn{2}{|c|}{ ILC1 } & \multicolumn{2}{|c|}{ ILC2 } & \multicolumn{2}{|c|}{ nGMM1 } & \multicolumn{5}{|c|}{ SM bkg. } \\
\hline & Sig. & Bkg. & Sig. & Bkg. & Sig. & Bkg. & $2 \mathrm{f}$ & $4 \mathrm{f}$ & $\mathrm{aa} \_2 \mathrm{f}$ & $a e \_3 f$ & aa_4f \\
\hline Preselection & 4895 & 15452 & 3705 & 11786 & 1427 & 7950 & 261999 & 1115296 & 87581 & 313496 & 14260 \\
\hline Lepton selection, $N_{\text {trk }}=2$ & 4532 & 3887 & 3436 & 3731 & 1325 & 3013 & 21615 & 294934 & 64989 & 112098 & 251 \\
\hline BeamCal veto & 4461 & 3814 & 3395 & 3681 & 1312 & 2978 & 20370 & 121784 & 64989 & 111493 & 233 \\
\hline$p_{T}>2.3 \mathrm{GeV}$ & 4348 & 3556 & 3060 & 3283 & 977 & 2508 & 19939 & 115883 & 62899 & 106535 & 133 \\
\hline$\left|\cos \theta_{\ell}\right|<0.95$ & 4067 & 3373 & 2997 & 3185 & 977 & 2477 & 13041 & 69986 & 62893 & 106527 & 102 \\
\hline$\Delta \phi<0.8$ & 2676 & 271 & 2024 & 292 & 868 & 193 & 11796 & 26636 & 42316 & 80441 & 11 \\
\hline$E_{\mathrm{vis}}<25 \mathrm{GeV}$ & 1939 & 180 & 1498 & 210 & 645 & 136 & 0.0 & 6569 & 84 & 105 & 11 \\
\hline$E_{\text {miss }}>300 \mathrm{GeV}$ & 1939 & 180 & 1498 & 210 & 645 & 136 & 0.0 & 5595 & 84 & 105 & 11 \\
\hline$\left|\cos \theta_{\text {miss }}\right|<0.98$ & 1939 & 180 & 1496 & 208 & 640 & 135 & 0.0 & 5574 & 77 & 105 & 11 \\
\hline$M_{\ell \ell}$ selection & 1939 & 176 & 1496 & 197 & 640 & 91 & 0.0 & 5506 & 77 & 100 & 9.6 \\
\hline
\end{tabular}

TABLE XXIX. Expected number of events for neutralino signal and major backgrounds for the electron final state and beam polarizations $\mathcal{P}\left(e^{-}, e^{+}\right)=(+0.8,-0.3)$. The integrated luminosity is assumed to be $500 \mathrm{fb}^{-1}$. For each benchmark model, the background refers to the other SUSY backgrounds.

\begin{tabular}{|c|c|c|c|c|c|c|c|c|c|c|c|}
\hline \multirow{2}{*}{$\begin{array}{l}\tilde{\chi}_{1}^{0} \tilde{\chi}_{2}^{0}\left(e^{-} e^{+}\right) \\
\mathcal{P}\left(e^{-}, e^{+}\right)=(+0.8,-0.3)\end{array}$} & \multicolumn{2}{|c|}{ ILC1 } & \multicolumn{2}{|c|}{ ILC2 } & \multicolumn{2}{|c|}{ nGMM1 } & \multicolumn{4}{|c|}{ SM bkg. } & \multirow[b]{2}{*}{ aa_4f } \\
\hline & Sig. & Bkg. & Sig. & Bkg. & Sig. & Bkg. & $2 f$ & $4 f$ & aa_2f & ae_3f & \\
\hline Preselection & 3486 & 6769 & 2495 & 5364 & 865 & 3144 & 166524 & 879484 & 87581 & 300006 & 14260 \\
\hline Lepton selection, $N_{\text {trk }}=2$ & 3214 & 1042 & 2308 & 1024 & 800 & 892 & 23031 & 348421 & 22592 & 38695 & 192 \\
\hline BeamCal veto & 3160 & 1024 & 2275 & 1014 & 794 & 880 & 17315 & 136363 & 22592 & 38347 & 176 \\
\hline$p_{T}>2.3 \mathrm{GeV}$ & 3047 & 938 & 2021 & 889 & 602 & 727 & 15819 & 126608 & 21961 & 36737 & 103 \\
\hline$\left|\cos \theta_{\ell}\right|<0.95$ & 2872 & 882 & 1985 & 852 & 602 & 694 & 8867 & 65285 & 21885 & 36359 & 82 \\
\hline$\Delta \phi<0.8$ & 1904 & 121 & 1382 & 169 & 541 & 184 & 3743 & 25036 & 14662 & 27658 & 34 \\
\hline$E_{\mathrm{vis}}<25 \mathrm{GeV}$ & 1284 & 69 & 1020 & 113 & 412 & 128 & 13 & 858 & 15 & 117 & 25 \\
\hline$E_{\text {miss }}>300 \mathrm{GeV}$ & 1284 & 69 & 1020 & 113 & 412 & 128 & 13 & 530 & 15 & 97 & 25 \\
\hline$\left|\cos \theta_{\text {miss }}\right|<0.98$ & 1284 & 69 & 1017 & 113 & 409 & 126 & 13 & 529 & 14 & 96 & 25 \\
\hline$M_{\ell \ell}$ selection & 1284 & 69 & 1017 & 111 & 409 & 119 & 13 & 508 & 14 & 83 & 19 \\
\hline
\end{tabular}

TABLE XXX. Expected number of events for neutralino signal and major backgrounds for the muon final state and beam polarizations $\mathcal{P}\left(e^{-}, e^{+}\right)=(+0.8,-0.3)$. The integrated luminosity is assumed to be $500 \mathrm{fb}^{-1}$. For each benchmark model, the background refers to the other SUSY backgrounds.

\begin{tabular}{|c|c|c|c|c|c|c|c|c|c|c|c|}
\hline \multirow{2}{*}{$\begin{array}{l}\tilde{\chi}_{1}^{0} \tilde{\chi}_{2}^{0}\left(\mu^{-} \mu^{+}\right) \\
\mathcal{P}\left(e^{-}, e^{+}\right)=(+0.8,-0.3) \\
\end{array}$} & \multicolumn{2}{|c|}{ ILC1 } & \multicolumn{2}{|c|}{ ILC2 } & \multicolumn{2}{|c|}{ nGMM1 } & \multicolumn{5}{|c|}{ SM bkg. } \\
\hline & Sig. & Bkg. & Sig. & Bkg. & Sig. & Bkg. & $2 \mathrm{f}$ & $4 \mathrm{f}$ & $\mathrm{aa} \_2 \mathrm{f}$ & $a e \_3 f$ & aa_4f \\
\hline Preselection & 3856 & 6399 & 2972 & 4887 & 1122 & 2887 & 166524 & 879484 & 87581 & 300006 & 14260 \\
\hline Lepton selection, $N_{\text {trk }}=2$ & 3573 & 950 & 2762 & 973 & 1039 & 844 & 20711 & 281254 & 64989 & 112161 & 251 \\
\hline BeamCal veto & 3513 & 932 & 2727 & 960 & 1028 & 835 & 19521 & 108836 & 64989 & 111489 & 233 \\
\hline$p_{T}>2.3 \mathrm{GeV}$ & 3421 & 869 & 2451 & 862 & 777 & 691 & 19069 & 103802 & 62899 & 106529 & 133 \\
\hline$\left|\cos \theta_{\ell}\right|<0.95$ & 3209 & 824 & 2402 & 836 & 777 & 681 & 11940 & 60407 & 62893 & 106521 & 102 \\
\hline$\Delta \phi<0.8$ & 2099 & 73 & 1633 & 93 & 692 & 72 & 10844 & 19083 & 42316 & 80441 & 11 \\
\hline$E_{\mathrm{vis}}<25 \mathrm{GeV}$ & 1522 & 50 & 1223 & 70 & 518 & 51 & 0.0 & 1213 & 84 & 105 & 11 \\
\hline$E_{\text {miss }}>300 \mathrm{GeV}$ & 1522 & 50 & 1223 & 70 & 518 & 51 & 0.0 & 686 & 84 & 105 & 11 \\
\hline$\left|\cos \theta_{\text {miss }}\right|<0.98$ & 1521 & 50 & 1222 & 69 & 516 & 51 & 0.0 & 678 & 77 & 105 & 11 \\
\hline$M_{\ell \ell}$ selection & 1521 & 49 & 1222 & 67 & 516 & 40 & 0.0 & 672 & 77 & 100 & 9.6 \\
\hline
\end{tabular}


[1] G. Aad et al. (ATLAS Collaboration), Phys. Lett. B 716, 1 (2012); S. Chatrchyan et al. (CMS Collaboration), Phys. Lett. B 716, 30 (2012).

[2] L. Susskind, Phys. Rev. D 20, 2619 (1979).

[3] M. Carena and H. E. Haber, Prog. Part. Nucl. Phys. 50, 63 (2003).

[4] N. Arkani-Hamed and S. Dimopoulos, J. High Energy Phys. 06 (2005) 073.

[5] L. J. Hall and Y. Nomura, J. High Energy Phys. 03 (2010) 076.

[6] H. Baer, V. Barger, P. Huang, D. Mickelson, A. Mustafayev, and X. Tata, Phys. Rev. D 87, 115028 (2013).

[7] J. R. Ellis, K. Enqvist, D. V. Nanopoulos, and F. Zwirner, Mod. Phys. Lett. A 01, 57 (1986).

[8] R. Barbieri and G. F. Giudice, Nucl. Phys. B306, 63 (1988).

[9] S. Dimopoulos and G. F. Giudice, Phys. Lett. B 357, 573 (1995).

[10] R. Kitano and Y. Nomura, Phys. Rev. D 73, 095004 (2006).

[11] M. Papucci, J. T. Ruderman, and A. Weiler, J. High Energy Phys. 09 (2012) 035.

[12] N. Arkani-Hamed, T. Han, M. Mangano, and L. T. Wang, Phys. Rep. 652, 1 (2016).

[13] For a review, see e.g. H. Baer and X. Tata, Weak Scale Supersymmetry: From Superfields to Scattering Events (Cambridge University Press, Cambridge, 2006), p. 537; S. P. Martin, A Supersymmetry Primer, Advanced Series on Directions in High Energy Physics Vol. 21 (World Scientific, Singapore, 2010), p. 1.

[14] H. Baer, V. Barger, and M. Savoy, Phys. Rev. D 93, 035016 (2016).

[15] H. Baer, V. Barger, and D. Mickelson, Phys. Rev. D 88, 095013 (2013).

[16] H. Baer, V. Barger, and S. Salam, Phys. Rev. Research 1, 023001 (2019).

[17] H. Baer, V. Barger, D. Mickelson, and M. PadeffkeKirkland, Phys. Rev. D 89, 115019 (2014); A. Mustafayev and X. Tata, Indian J. Phys. 88, 991 (2014).

[18] H. Baer, V. Barger, P. Huang, A. Mustafayev, and X. Tata, Phys. Rev. Lett. 109, 161802 (2012).

[19] G. G. Ross, K. Schmidt-Hoberg, and F. Staub, Phys. Lett. B 759, 110 (2016).

[20] S. P. Martin, Phys. Rev. D 61, 035004 (2000).

[21] H. Baer, V. Barger, and D. Sengupta, Phys. Rev. D 98, 015039 (2018).

[22] H. Baer, V. Barger, J. S. Gainer, D. Sengupta, H. Serce, and X. Tata, Phys. Rev. D 98, 075010 (2018).

[23] H. Baer, A. Mustafayev, and X. Tata, Phys. Rev. D 90, 115007 (2014).

[24] H. Baer, V. Barger, D. Mickelson, A. Mustafayev, and X. Tata, J. High Energy Phys. 06 (2014) 172.

[25] H. Baer, V. Barger, J. S. Gainer, P. Huang, M. Savoy, D. Sengupta, and X. Tata, Eur. Phys. J. C 77, 499 (2017).

[26] D. Matalliotakis and H. P. Nilles, Nucl. Phys. B435, 115 (1995); P. Nath and R. L. Arnowitt, Phys. Rev. D 56, 2820 (1997); J. R. Ellis, T. Falk, K. A. Olive, and Y. Santoso, Nucl. Phys. B652, 259 (2003); H. Baer, A. Mustafayev, S. Profumo, A. Belyaev, and X. Tata, J. High Energy Phys. 07 (2005) 065.

[27] H. Baer, V. Barger, H. Serce, and X. Tata, Phys. Rev. D 94, 115017 (2016).
[28] H. Abramowicz et al., The International Linear Collider Technical Design Report - Volume 4: Detectors (2013), arXiv:1306.6329.

[29] P. Bechtle, K. Desch, and P. Wienemann, Comput. Phys. Commun. 174, 47 (2006).

[30] H. Baer, V. Barger, A. Lessa, and X. Tata, Phys. Rev. D 86, 117701 (2012).

[31] ATLAS Collaboration, arXiv:1307.7292.

[32] A. M. Sirunyan et al. (CMS Collaboration), Phys. Lett. B 782, 440 (2018).

[33] M. Aaboud et al. (ATLAS Collaboration), Phys. Rev. D 97, 052010 (2018).

[34] K. J. Bae, H. Baer, and E. J. Chun, Phys. Rev. D 89, 031701 (2014).

[35] H. Baer, V. Barger, M. Savoy, and X. Tata, Phys. Rev. D 94, 035025 (2016).

[36] H. Baer, V. Barger, P. Huang, D. Mickelson, A. Mustafayev, W. Sreethawong, and X. Tata, Phys. Rev. Lett. 110, 151801 (2013).

[37] K. Choi, A. Falkowski, H. P. Nilles, M. Olechowski, and S. Pokorski, J. High Energy Phys. 11 (2004) 076.

[38] K. Choi, A. Falkowski, H. P. Nilles, and M. Olechowski, Nucl. Phys. B718, 113 (2005).

[39] K. Choi and H. P. Nilles, J. High Energy Phys. 04 (2007) 006.

[40] W. Kilian, T. Ohl, and J. Reuter, Eur. Phys. J. C 71, 1742 (2011).

[41] T. Behnke et al., The International Linear Collider Technical Design Report - Volume 1: Executive Summary (2013), arXiv:1306.6327.

[42] D. Schulte, CERN Reports No. CERN-PS-99-014-LP and No. CLIC-NOTE-387, 1999, https://cds.cern.ch/record/ 382453.

[43] T. Barklow, J. Brau, K. Fujii, J. Gao, J. List, N. Walker, and K. Yokoya, arXiv:1506.07830.

[44] H. Abramowicz et al., J. Instrum. 5, P12002 (2010).

[45] iLCSoft Home Page (2016), https://ilcsoft.desy.de/portal.

[46] P. Mora de Freitas and H. Videau, in Linear colliders, Proceedings, International Workshop on Physics and Experiments with Future Electron-Positron Linear Colliders, LCWS 2002, Seogwipo, Jeju Island, Korea (DESY, 2002), pp. 623-627, LC-TOOL-2003-010.

[47] F. Gaede, Nucl. Instrum. Methods Phys. Res., Sect. A 559, 177 (2006).

[48] M. A. Thomson, Nucl. Instrum. Methods Phys. Res., Sect. A 611, 25 (2009).

[49] M. Tanabashi et al. (Particle Data Group), Phys. Rev. D 98, 030001 (2018).

[50] F. Paige, S. Protopopescu, H. Baer, and X. Tata, ISAJET 7.85 A Monte Carlo Event Generator for $p p, \bar{p} p$, and $e^{+} e^{-}$ Reactions, arXiv:hep-ph/0312045.

[51] W. Porod, Comput. Phys. Commun. 153, 275 (2003).

[52] S.-L. Lehtinen, Ph.D. thesis, Hamburg University, 2018, Report No. DESY-THESIS-2018-035, https://doi.org/ 10.3204/PUBDB-2018-04509.

[53] J. Yan, S. Watanuki, K. Fujii, A. Ishikawa, D. Jeans, J. Strube, J. Tian, and H. Yamamoto, Phys. Rev. D 94, 113002 (2016).

[54] K. Fujii et al., arXiv:1506.05992.

[55] M. Frank, T. Hahn, S. Heinemeyer, W. Hollik, H. Rzehak, and G. Weiglein, J. High Energy Phys. 02 (2007) 047; 
G. Degrassi, S. Heinemeyer, W. Hollik, P. Slavich, and G. Weiglein, Eur. Phys. J. C 28, 133 (2003); S. Heinemeyer, W. Hollik, and G. Weiglein, Eur. Phys. J. C 9, 343 (1999); Comput. Phys. Commun. 124, 76 (2000).

[56] P. Bambade et al., arXiv:1903.01629.

[57] S. Caiazza, Ph.D. thesis, Universität Hamburg, 2018, Report No. DESY-THESIS-2018-037, https://doi.org/10.3204/ PUBDB-2018-05289.

[58] S. Catani, Y. L. Dokshitzer, M. H. Seymour, and B. R. Webber, Nucl. Phys. B406, 187 (1993).

[59] S. D. Ellis and D. E. Soper, Phys. Rev. D 48, 3160 (1993).

[60] S. Catani, Y. L. Dokshitzer, M. Olsson, G. Turnock, and B. R. Webber, Phys. Lett. B 269, 432 (1991).

[61] M. Berggren, F. Brümmer, J. List, G. Moortgat-Pick, T. Robens, K. Rolbiecki, and H. Sert, Eur. Phys. J. C 73, 2660 (2013).

[62] D. M. Pierce, J. A. Bagger, K. T. Matchev, and R. j. Zhang, Nucl. Phys. B491, 3 (1997).

[63] See Chap 2.3.3., page 30/31 and Chap 6.6.2, Fig 6.11, page 118/119 of [70].

[64] K. J. Bae, H. Baer, N. Nagata, and H. Serce, Phys. Rev. D 94, 035015 (2016).

[65] H. Baer, A. Mustafayev, S. Profumo, A. Belyaev, and X. Tata, Phys. Rev. D 71, 095008 (2005).
[66] E. A. Baltz, M. Battaglia, M. E. Peskin, and T. Wizansky, Phys. Rev. D 74, 103521 (2006).

[67] H. Baer, C. Balazs, and A. Belyaev, J. High Energy Phys. 03 (2002) 042.

[68] H. Baer, A. Belyaev, T. Krupovnickas, and J. O'Farrill, J. Cosmol. Astropart. Phys. 08 (2004) 005.

[69] H. Baer, V. Barger, D. Sengupta, and X. Tata, Eur. Phys. J. C 78, 838 (2018).

[70] K. J. Bae, H. Baer, A. Lessa, and H. Serce, J. Cosmol. Astropart. Phys. 10 (2014) 082.

[71] G. Belanger, F. Boudjema, A. Pukhov, and A. Semenov, Comput. Phys. Commun. 149, 103 (2002).

[72] N. Nguyen, D. Horns, and T. Bringmann, AstroFit: An Interface Program for Exploring Complementarity in Dark Matter Research, Astroparticle, Particle, Space Physics, Radiation Interaction, Detectors and Medical Physics Applications (World Scientific, Singapore, 2012), pp. 1025-1030, https://doi.org/10.1142/9789814405072_0154.

[73] P. A. R. Ade et al. (Planck Collaboration), Astron. Astrophys. 594, A13 (2016).

[74] G. A. Blair, W. Porod, and P. M. Zerwas, Phys. Rev. D 63, 017703 (2000).

[75] G. A. Blair, W. Porod, and P. M. Zerwas, Eur. Phys. J. C 27, 263 (2003). 